\title{
EQUIDISTRIBUTION AND COUNTING FOR ORBITS OF GEOMETRICALLY FINITE HYPERBOLIC GROUPS
}

\author{
HEE OH AND NIMISH A. SHAH
}

\section{Contents}

1. Introduction

2. Transverse measures

3. Equidistribution of $\mathcal{G}_{*}^{r} \mu_{E}^{\mathrm{Leb}}$

4. Geometric finiteness of closed totally geodesic immersions

5. On the cuspidal neighborhoods of $\Lambda_{\mathrm{bp}}(\Gamma) \cap \partial \tilde{S}$

6. Parabolic corank and criterion for finiteness of $\mu_{E}^{\mathrm{PS}}$

7. Orbital counting for discrete hyperbolic groups

8. Appendix: Equality of two Haar measures

Acknowledgements

References

\section{INTRODUCTION}

1.1. Motivation and overview. Let $G$ denote the identity component of the special orthogonal group $\mathrm{SO}(n, 1), n \geq 2$, and $V$ a finite-dimensional real vector space on which $G$ acts linearly from the right.

A discrete subgroup of a locally compact group with finite covolume is called a lattice. For $v \in V$ and a subgroup $H$ of $G$, let $H_{v}=\{h \in H: v h=v\}$ denote the stabilizer of $v$ in $H$.

A subgroup $H$ of $G$ is called symmetric if there exists a non-trivial involutive automorphism $\sigma$ of $G$ such that the identity component of $H$ is the same as the identity component of $G^{\sigma}=\{g \in G: \sigma(g)=g\}$.

Theorem 1.1 (Duke-Rudnick-Sarnak [9]). Fix $w_{0} \in V$ such that $G_{w_{0}}$ is symmetric. Let $\Gamma$ be a lattice in $G$ such that $\Gamma_{w_{0}}$ is a lattice in $G_{w_{0}}$. Then for any norm $\|\cdot\|$ on $V$,

$$
\lim _{T \rightarrow \infty} \frac{\#\left\{w \in w_{0} \Gamma:\|w\|<T\right\}}{\operatorname{vol}\left(B_{T}\right)}=\frac{\operatorname{vol}\left(\Gamma_{w_{0}} \backslash G_{w_{0}}\right)}{\operatorname{vol}(\Gamma \backslash G)}
$$

Received by the editors April 7, 2011 and, in revised form, January 27, 2012, and May 31, 2012 .

2010 Mathematics Subject Classification. Primary 11N45, 37F35, 22E40; Secondary 37A17, $20 \mathrm{~F} 67$.

Key words and phrases. Geometrically finite hyperbolic groups, mixing of geodesic flow, totally geodesic submanifolds, Patterson-Sullivan measure.

The first author was supported in part by NSF Grants \#0629322 and \#1068094.

The second author was supported in part by NSF Grant \#1001654. 
where $B_{T}:=\left\{w \in w_{0} G:\|w\|<T\right\}$ and the volumes on $G_{w_{0}}, G$ and $w_{0} G \simeq G_{w_{0}} \backslash G$ are computed with respect to the right invariant measures chosen compatibly.

Eskin and McMullen 10 gave a simpler proof of Theorem 1.1 based on the mixing property of the geodesic flow of a hyperbolic manifold with finite volume. It may be noted that this approach for counting via mixing was used earlier by Margulis in his 1970 thesis [20]. We also refer to [3] for a quantitative version of Theorem 1.1.

The group $G$ can be considered as the group of orientation preserving isometries of the $n$-dimensional hyperbolic space $\mathbb{H}^{n}$. The main achievement of this paper lies in extending Theorem 1.1 to a suitable class of discrete subgroups $\Gamma$ of infinite covolume in $G$; namely, the groups $\Gamma$ with finite Bowen-Margulis-Sullivan measure $m^{\text {BMS }}$ on $\Gamma \backslash \mathbb{H}^{n}$. In particular, this class contains all geometrically finite subgroups of $G$. The analogue of $\operatorname{vol}\left(\Gamma_{w_{0}} \backslash G_{w_{0}}\right)$ turns out to be a very interesting quantity, which we will call the 'skinning size' of $w_{0}$ relative to $\Gamma$ and denote by $\operatorname{sk}_{\Gamma}\left(w_{0}\right)$. In fact, $\operatorname{sk}_{\Gamma}\left(w_{0}\right)$ will be the total mass of a Patterson-Sullivan type measure on the unit normal bundle of a closed immersed submanifold of $\Gamma \backslash \mathbb{H}^{n}$ associated to $G_{w_{0}}$. One of the important components of this work is to completely determine when $\operatorname{sk}_{\Gamma}\left(w_{0}\right)$ is finite (Theorem 1.5). In particular, $\operatorname{sk}_{\Gamma}\left(w_{0}\right)<\infty$ for any geometrically finite $\Gamma$ whose critical exponent $\delta$ is greater than the codimension of the associated submanifold.

The main ergodic theoretic ingredient in the proof is the description for the limiting distribution of the evolution of the smooth measure on the unit normal bundle of a closed totally geodesically immersed submanifold of $\Gamma \backslash \mathbb{H}^{n}$ under the geodesic flow. The corresponding equidistribution statement (Theorem 1.8) is applicable to many other problems. For example, in [23, 24] it has been applied to the study of the asymptotic distributions in circle packings in the Euclidean plane or a sphere, invariant under a non-elementary group of Möbius transformations.

1.2. Statement of main result. Our generalization of Theorem 1.1 for discrete subgroups which are not necessarily lattices involves terms which can be best explained in the language of hyperbolic geometry. Let $\Gamma<G$ be a torsion-free discrete subgroup which is non-elementary, that is, $\Gamma$ has no abelian subgroup of finite index. This is a standing assumption on $\Gamma$ throughout the whole paper. Now $\Gamma$ acts properly discontinuously on $\mathbb{H}^{n}$. Let $0<\delta \leq n-1$ be the critical exponent of $\Gamma$ (see 3.1.1). Let $\left\{\nu_{x}\right\}_{x \in \mathbb{H}^{n}}$ be a $\Gamma$-invariant conformal density of dimension $\delta$ on the geometric boundary $\partial \mathbb{H}^{n}$ (see (2.11)) which exists by Patterson [26] and Sullivan [36]. Let $m^{\text {BMS }}$ denote the Bowen-Margulis-Sullivan measure on the unit tangent bundle $\mathrm{T}^{1}\left(\Gamma \backslash \mathbb{H}^{n}\right)$ associated to $\left\{\nu_{x}\right\}$ (see (3.2)).

For $u \in \mathrm{T}^{1}\left(\mathbb{H}^{n}\right)$, we denote by $u^{ \pm} \in \partial \mathbb{H}^{n}$ the forward and the backward endpoints of the geodesic determined by $u$, respectively, and by $\pi(u) \in \mathbb{H}^{n}$ the base point of $u$. Let $\mathbf{p}: \mathrm{T}^{1}\left(\mathbb{H}^{n}\right) \rightarrow \mathrm{T}^{1}\left(\Gamma \backslash \mathbb{H}^{n}\right)$ be the canonical quotient map.

Let $V$ be a finite-dimensional vector space on which $G$ acts linearly. Let $w_{0} \in V$ be such that $G_{w_{0}}$ is a symmetric subgroup or the stabilizer $G_{\mathbb{R} w_{0}}$ of the line $\mathbb{R} w_{0}$ is a parabolic subgroup. We define a subset $\tilde{E} \subset \mathrm{T}^{1}\left(\mathbb{H}^{n}\right)$ associated to the orbit $w_{0} \Gamma$ in each case.

When $G_{w_{0}}$ is a symmetric subgroup associated to an involution $\sigma$, choose a Cartan involution $\theta$ of $G$ which commutes with $\sigma$, and let $o \in \mathbb{H}^{n}$ be such that its stabilizer $G_{o}$ is the fixed group of $\theta$. Then $\tilde{S}:=G_{w_{0}} . o$ is an isometric imbedding 
of $\mathbb{H}^{k}$ in $\mathbb{H}^{n}$ for some $0 \leq k \leq n-1$, where the embeddings of $\mathbb{H}^{0}$ and $\mathbb{H}^{1}$ mean a point and a complete geodesic, respectively. Let $\tilde{E} \subset \mathrm{T}^{1}\left(\mathbb{H}^{n}\right)$ be the unit normal bundle of $\tilde{S}$.

In the case when $G_{\mathbb{R} w_{0}}$ is parabolic, we fix any $o \in \mathbb{H}^{n}$. If $N$ is the unipotent radical of $G_{\mathbb{R} w_{0}}$, then $\tilde{S}:=N . o$ is a horosphere. We set $\tilde{E} \subset \mathrm{T}^{1}\left(\mathbb{H}^{n}\right)$ to be the unstable horosphere over $\tilde{S}$.

Now in either case, we define the following Borel measure on $\tilde{E}$ :

$$
d \mu_{\tilde{E}}^{\mathrm{PS}}(v):=e^{\delta \beta_{v}+(x, \pi(v))} d \nu_{x}\left(v^{+}\right)
$$

for $x \in \mathbb{H}^{n}$, and $\beta_{\xi}\left(x_{1}, x_{2}\right)$ denotes the value of the Busemann function, that is, the signed distance between the horospheres based at $\xi$, one passing through $x_{1}$ and the other through $x_{2}$ (see (2.2) $)$. This definition of $\mu_{\tilde{E}}^{\mathrm{PS}}$ is independent of the choice of $x \in \mathbb{H}^{n}$. Due to the $\Gamma$-invariance property of the conformal density $\left\{\nu_{x}\right\}$, it induces a measure on $E:=\mathbf{p}(\tilde{E})$ which we denote by $\mu_{E}^{\mathrm{PS}}$.

Fix any $X_{0} \in \tilde{E}$ based at $o$, and let $A=\left\{a_{r}: r \in \mathbb{R}\right\}$ be a one-parameter subgroup of $G$ consisting of $\mathbb{R}$-diagonalizable elements such that $r \mapsto a_{r}$. $X_{0}$ is a unit speed geodesic. Note that $A$ is contained in a copy of $\operatorname{SO}(2,1) \cong \operatorname{PSL}(2, \mathbb{R})$ such that $a_{r}$ corresponds to $d_{r}=\operatorname{diag}\left(e^{r / 2}, e^{-r / 2}\right)$. Any irreducible representation of $\operatorname{PSL}(2, \mathbb{R})$ is given by the standard action of $\operatorname{SL}(2, \mathbb{R})$ on homogeneous polynomials of degree $k$ in two variables such that the action of $-I$ is trivial, so $k$ is even and the largest eigenvalue of $d_{r}$ is $e^{(k / 2) r}$. Therefore, if $\lambda$ denotes the log of the largest eigenvalue of $a_{1}$ on $\mathbb{R}-\operatorname{span}\left(w_{0} G\right)$, then $\lambda \in \mathbb{N}$. We set

$$
w_{0}^{\lambda}:=\lim _{r \rightarrow \infty} e^{-\lambda r} w_{0} a_{r} \neq 0, \quad \text { by [13, Lemma 4.2]. }
$$

Theorem 1.2. Let $\Gamma<G$ be a non-elementary discrete subgroup with $\left|m^{\mathrm{BMS}}\right|<\infty$. Suppose that $w_{0} \Gamma$ is discrete and that its skinning size $\operatorname{sk}_{\Gamma}\left(w_{0}\right):=\left|\mu_{E}^{\mathrm{PS}}\right|$ is finite. Then for any $G_{O}$-invariant norm $\|\cdot\|$ on $V$, we have

$$
\lim _{T \rightarrow \infty} \frac{\#\left\{w \in w_{0} \Gamma:\|w\|<T\right\}}{T^{\delta / \lambda}}=\frac{\left|\nu_{o}\right| \cdot \operatorname{sk}_{\Gamma}\left(w_{0}\right)}{\delta \cdot\left|m^{\mathrm{BMS}}\right| \cdot\left\|w_{0}^{\lambda}\right\|^{\delta / \lambda}} .
$$

Remark 1.3. (1) If $\Gamma$ is convex cocompact, $\operatorname{sk}_{\Gamma}\left(w_{0}\right)<\infty$. In the case when $G_{\mathbb{R} w_{0}}$ is parabolic, $\operatorname{sk}_{\Gamma}\left(w_{0}\right)<\infty$ as well. A finiteness criterion for $\operatorname{sk}_{\Gamma}\left(w_{0}\right)$ is provided in $\S 1.4$.

(2) Since $w_{0} \Gamma$ is infinite, $\operatorname{sk}_{\Gamma}\left(w_{0}\right)>0$ (Proposition 6.7), and hence the limit (1.1) is strictly positive.

(3) The description of the limit changes if we do not assume the $G_{o}$-invariance of the norm $\|\cdot\|$; see Theorem 7.8, Remark 7.9)(3)-(5), and Theorem 7.10.

(4) If $G_{w_{0}}$ is symmetric and $\Gamma$ is Zariski dense in $G$, then the condition $\left|\mu_{E}^{\mathrm{PS}}\right|<\infty$ implies that $w_{0} \Gamma$ is discrete, for by Theorem 2.21 and Remark 2.22, $w_{0} \Gamma$ is closed in $w_{0} G$, and by [13, Lemma 4.2], $w_{0} G$ is closed in $V$. Therefore $w_{0} \Gamma$ is closed and hence discrete in $V$.

(5) If $G_{\mathbb{R} w_{0}}$ is parabolic, then the condition $\left|\mu_{E}^{\mathrm{PS}}\right|<\infty$ implies that $w_{0} \Gamma$ is discrete. To see this, note that if the horosphere $\tilde{S}$ is based at $\xi$, then $\partial \tilde{S}=\{\xi\}$, and by Theorem 2.21, $\Gamma \tilde{S}$ is closed in $\mathbb{H}^{n}$ and $w_{0} \Gamma$ is closed in $w_{0} G=\overline{w_{0} G} \backslash\{0\}$. If $w_{0} \Gamma$ is not closed in $V, w_{0} \gamma_{i} \rightarrow 0$ for a sequence $\left\{\gamma_{i}\right\} \subset \Gamma$. Then $\gamma_{i} o \rightarrow \xi$ 


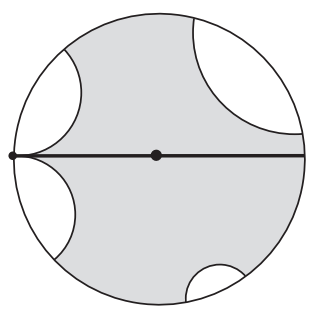

FiguRE 1. An externally $\Gamma$-parabolic vector

and $\xi$ is a horospherical limit point of $\Gamma$. Since $\left|m^{\mathrm{BMS}}\right|$ is finite, the geodesic flow is mixing (Theorem 3.2), and hence by [7, Thm. A and Prop. B], $\Gamma \tilde{S}$ is dense in $\pi\left(\left\{u: u^{-} \in \Lambda(\Gamma)\right\}\right)$, a contradiction to $\Gamma \tilde{S}$ being closed. Therefore $w_{0} \Gamma$ is closed and hence discrete in $V$.

Thanks are due to the referee for the last two remarks.

A discrete group $\Gamma$ is called geometrically finite if the unit neighborhood of its convex core 1 has finite Riemannian volume (see also Theorem 4.6). Any discrete group admitting a finite sided polyhedron as a fundamental domain in $\mathbb{H}^{n}$ is geometrically finite.

Sullivan [36] showed that $\left|m^{\mathrm{BMS}}\right|<\infty$ for all geometrically finite $\Gamma$. However, Theorem 1.2 is not limited to those, as Peigné [27] constructed a large class of geometrically infinite groups admitting a finite Bowen-Margulis-Sullivan measure.

We will provide a general criterion on the finiteness of $\operatorname{sk}_{\Gamma}\left(w_{0}\right)$ in Theorem 1.14. For the sake of concreteness, we first describe the results for the standard representation of $G$.

1.3. Standard representation of $G$. Let $Q$ be a real quadratic form of signature $(n, 1)$ for $n \geq 2$ and $G$ the identity component of the special orthogonal group $\operatorname{SO}(Q)$. Then $G$ acts on $\mathbb{R}^{n+1}$ by the matrix multiplication from the right, i.e., the standard representation. For any non-zero $w_{0} \in \mathbb{R}^{n+1}$, up to conjugation and commensurability, $G_{w_{0}}$ is $\mathrm{SO}(n-1,1)$ (resp. $\left.\mathrm{SO}(n)\right)$ if $Q\left(w_{0}\right)>0$ (resp. if $Q\left(w_{0}\right)<$ $0)$. If $Q\left(w_{0}\right)=0$, the stabilizer of the line $\mathbb{R} w_{0}$ is a parabolic subgroup. Therefore Theorem 1.2 is applicable for any non-zero $w_{0} \in \mathbb{R}^{n+1}$, provided $\operatorname{sk}_{\Gamma}\left(w_{0}\right)<\infty$ (in this case, $\lambda=1$ ).

An element $\gamma \in \Gamma$ is called parabolic if there exists a unique fixed point of $\gamma$ in $\partial \mathbb{H}^{n}$. For $\xi \in \partial \mathbb{H}^{n}$, we denote by $\Gamma_{\xi}$ the stabilizer of $\xi$ in $\Gamma$ and call $\xi$ a parabolic fixed point of $\Gamma$ if $\xi$ is fixed by a parabolic element of $\Gamma$.

Noting that $G_{w_{0}}$ is the isometry group of the codimension one totally geodesic subspace, say, $\tilde{S}_{w_{0}}$, when $Q\left(w_{0}\right)>0$, we give the following:

Definition 1.4. Let $w_{0} \Gamma$ be discrete. Then $w_{0} \in \mathbb{R}^{n+1}$ is said to be externally $\Gamma$-parabolic if $Q\left(w_{0}\right)>0$ and there exists a parabolic fixed point $\xi \in \partial \tilde{S}_{w_{0}}$ for $\Gamma$ such that $G_{w_{0}} \cap \Gamma_{\xi}$ is trivial, where $\partial \tilde{S}_{w_{0}} \subset \partial \mathbb{H}^{n}$ denotes the boundary of $\tilde{S}_{w_{0}}$ in $\overline{\mathbb{H}^{n}}$.

\footnotetext{
${ }^{1}$ The convex core $C_{\Gamma} \subset \Gamma \backslash \mathbb{H}^{n}$ of $\Gamma$ is the image of the minimal convex subset of $\mathbb{H}^{n}$ which contains all geodesics connecting any two points in the limit set of $\Gamma$.
} 
For $n=2, w_{0} \in \mathbb{R}^{3}$ with $Q\left(w_{0}\right)>0$ is externally $\Gamma$-parabolic if and only if the projection of the geodesic $\tilde{S}_{w_{0}}$ in $\Gamma \backslash \mathbb{H}^{n}$ is divergent in both directions, and at least one end of $\tilde{S}_{w_{0}}$ goes into a cusp of a fundamental domain of $\Gamma$ in $\mathbb{H}^{2}$ (see Figure 1).

Theorem 1.5 (On the finiteness of $\operatorname{sk}_{\Gamma}\left(w_{0}\right)$ ). Let $\Gamma$ be geometrically finite and $w_{0} \Gamma$ be discrete.

(1) If $\delta>1$, then $\operatorname{sk}_{\Gamma}\left(w_{0}\right)<\infty$.

(2) If $\delta \leq 1$, then $\operatorname{sk}_{\Gamma}\left(w_{0}\right)=\infty$ if and only if $w_{0}$ is externally $\Gamma$-parabolic.

Corollary 1.6. Let $\Gamma$ be geometrically finite and $w_{0} \Gamma$ discrete. If either $\delta>1$ or $w_{0}$ is not externally $\Gamma$-parabolic, then (1.1) holds.

Remark 1.7. (1) For geometrically finite $\Gamma$, if the Riemannian volume of $E$ is finite, then $\operatorname{sk}_{\Gamma}\left(w_{0}\right)<\infty$ (Corollary 1.15).

(2) It can be proved that if $\delta \leq 1$ and $w_{0}$ is externally $\Gamma$-parabolic, the asymptotic count is of the order $T \log T$ if $\delta=1$ and of the order $T$ if $\delta<1$, instead of $\mathrm{T}^{\delta}$ (cf. [25]).

(3) When $Q\left(w_{0}\right)<0$, the orbital counting with respect to the hyperbolic metric balls was obtained by Lax and Phillips [19] for $\Gamma$ geometrically finite with $\delta>$ $(n-1) / 2$, by Lalley [18 for convex cocompact subgroups and by Roblin 31] for all groups with finite Bowen-Margulis-Sullivan measure.

(4) When $Q\left(w_{0}\right)=0$ and $\Gamma$ is geometrically finite with $\delta>(n-1) / 2$, a version of Theorem 1.2 was obtained in [17.

1.4. Equidistribution of expanding submanifolds. In this section, we will describe the main ergodic theoretic ingredients used in the proof of Theorem 1.2, Let $\tilde{E} \subset \mathrm{T}^{1}\left(\mathbb{H}^{n}\right)$ be one of the following:

(1) an unstable horosphere over a horosphere $\tilde{S}$ in $\mathbb{H}^{n}$;

(2) the unit normal bundle of a complete proper connected totally geodesic subspace $\tilde{S}$ of $\mathbb{H}^{n}$; that is, $\tilde{S}$ is an isometric imbedding of $\mathbb{H}^{k}$ in $\mathbb{H}^{n}$ for some $0 \leq k \leq n-1$.

Let $\Gamma$ be a discrete subgroup of $G$, and set $E:=\mathbf{p}(\tilde{E})$ for the projection $\mathbf{p}$ : $\mathrm{T}^{1}\left(\mathbb{H}^{n}\right) \rightarrow \mathrm{T}^{1}\left(\Gamma \backslash \mathbb{H}^{n}\right)$.

Recall that $\left\{\nu_{x}: x \in \mathbb{H}^{n}\right\}$ denotes a Patterson-Sullivan density of dimension $\delta$. Let $\left\{m_{x}: x \in \mathbb{H}^{n}\right\}$ denote a $G$-invariant conformal density of dimension $(n-1)$. We consider the following locally finite Borel measures on $\tilde{E}$ :

$$
d \mu_{\tilde{E}}^{\mathrm{Leb}}(v)=e^{(n-1) \beta_{v^{+}}(o, \pi(v))} d m_{o}\left(v^{+}\right), \quad d \mu_{\tilde{E}}^{\mathrm{PS}}(v)=e^{\delta \beta_{v^{+}}(o, \pi(v))} d \nu_{o}\left(v^{+}\right),
$$

where $o \in \mathbb{H}^{n}$. Note that $\mu_{\tilde{E}}^{\text {Leb }}$ is the measure associated to the Riemannian volume form on $\tilde{E}$.

The measures $\mu_{\tilde{E}}^{\mathrm{PS}}$ and $\mu_{\tilde{E}}^{\mathrm{Leb}}$ are invariant under $\Gamma_{\tilde{E}}=\{\gamma \in \Gamma: \gamma(\tilde{E})=\tilde{E}\}$ and hence induce measures on $\Gamma_{\tilde{E}} \backslash \tilde{E}$. We denote by $\mu_{E}^{\mathrm{Leb}}$ and $\mu_{E}^{\mathrm{PS}}$, respectively, the projections of these measures on $E$ via the projection map $\Gamma_{\tilde{E}} \backslash \tilde{E} \rightarrow E$ induced by p.

Let $m^{\mathrm{BR}}$ denote the Burger-Roblin measure on $\mathrm{T}^{1}\left(\Gamma \backslash \mathbb{H}^{n}\right)$ associated to the conformal densities $\left\{\nu_{x}\right\}$ in the backward direction and $\left\{m_{x}\right\}$ in the forward direction (6], 31, and see (3.3)).

Let $\left\{\mathcal{G}^{t}\right\}$ denote the geodesic flow on $\mathrm{T}^{1}\left(\mathbb{H}^{n}\right)$. 
Theorem 1.8. Suppose that $\left|m^{\mathrm{BMS}}\right|<\infty$ and $\left|\mu_{E}^{\mathrm{PS}}\right|<\infty$. Let $F \subset E$ be a Borel subset with $\mu_{E}^{\mathrm{PS}}(\partial F)=0$. For any $\psi \in C_{c}\left(\mathrm{~T}^{1}\left(\Gamma \backslash \mathbb{H}^{n}\right)\right)$,

$$
\lim _{t \rightarrow+\infty} e^{(n-1-\delta) t} \cdot \int_{F} \psi\left(\mathcal{G}^{t}(v)\right) d \mu_{E}^{\mathrm{Leb}}(v)=\frac{\mu_{E}^{\mathrm{PS}}(F)}{\left|m^{\mathrm{BMS}}\right|} \cdot m^{\mathrm{BR}}(\psi) .
$$

In particular, this holds for $F=E$.

See Theorem 3.6 for a version of Theorem 1.8 without the finiteness assumption on $\left|\mu_{E}^{\mathrm{PS}}\right|$.

Remark 1.9. Theorem [1.8 applies to $F$ with $\mu_{E}^{\mathrm{Leb}}(F)=\infty$ as well, provided $\left|\mu_{E}^{\mathrm{PS}}\right|<\infty$. The proof for this generality requires greater care since it cannot be deduced from the cases of $F$ bounded. It is precisely this general nature of our equidistribution theorem which enabled us to state Theorem 1.2 for general groups $\Gamma$, only assuming the finiteness of the skinning size $\operatorname{sk}_{\Gamma}\left(w_{0}\right)=\left|\mu_{E}^{\mathrm{PS}}\right|$ for a suitable E.

When $E$ is a horosphere and $F$ is bounded, Theorem 1.8 was obtained earlier by Roblin [31, p. 52]. We were motivated to formulate and prove the result from an independent view point; our attention was especially on the case of $\pi(E)$ being a totally geodesic immersion. This case involves many new features, observations, and applications (cf. 23], 24]). The main key to our proof is the transversality theorem, Theorem 3.5 which was influenced by the work of Schapira 34. The transversality theorem provides a precise relation between the transversal intersections of geodesic evolution of $F$ with a given piece, say $T$, of a weak stable leaf and the transversal measure corresponding to the $m^{\mathrm{BMS}}$ measure on $T$.

For $\Gamma$ Zariski dense, we generalize Theorem 1.8 to $\psi \in C_{c}(\Gamma \backslash G)$. To state the generalization, we fix $o \in \mathbb{H}^{n}$ and $X_{0} \in \tilde{E}$ based at $o$. Then, for $K=G_{o}$ and $M=G_{X_{0}}$, we may identify $\mathbb{H}^{n}$ and $\mathrm{T}^{1}\left(\mathbb{H}^{n}\right)$ with $G / K$ and $G / M$, respectively. Let $A=\left\{a_{r}\right\}$ be the one-parameter subgroup such that the right translation action by $a_{r}$ on $G / M$ corresponds to $\mathcal{G}^{r}$. Let $\bar{m}^{\mathrm{BR}}$ denote the measure on $\Gamma \backslash G$ which is the $M$-invariant extension of $m^{\mathrm{BR}}$ via the natural projection map $\Gamma \backslash G \rightarrow \Gamma \backslash G / M=$ $\mathrm{T}^{1}\left(\Gamma \backslash \mathbb{H}^{n}\right)$. Let $H=G_{\tilde{E}}$, and let $d h$ denote the invariant measure on $\Gamma_{H} \backslash H$ whose projection to $E$ coincides with $\mu_{E}^{\mathrm{Leb}}$.

Theorem 1.10. Let $\Gamma$ be a Zariski dense discrete subgroup of $G$ such that $\left|m^{\mathrm{BMS}}\right|<$ $\infty$ and $\left|\mu_{E}^{\mathrm{PS}}\right|<\infty$. Then for any $\psi \in C_{c}(\Gamma \backslash G)$,

$$
\lim _{r \rightarrow \infty} e^{(n-1-\delta) r} \int_{h \in \Gamma_{H} \backslash H} \psi\left(\Gamma h a_{r}\right) d h=\frac{\left|\mu_{E}^{\mathrm{PS}}\right|}{\left|m^{\mathrm{BMS}}\right|} \bar{m}^{\mathrm{BR}}(\psi) .
$$

When $\Gamma$ is a lattice in $G$ and $E$ is of finite Riemannian volume, Theorem 1.10 is due to Sarnak 33 for horocycles in $\mathbb{H}^{2}$, Randol 28] for unit normal vectors based at a point in the cocompact lattice case in $\mathbb{H}^{2}$, and Duke-Rudnick-Sarnak 9] and Eskin-McMullen [10] in general (also see [15, Appendix]).

In 87, we deduce Theorem 1.2 from Theorem 1.8. The standard techniques of orbital counting via equidistribution results require significant modifications due to the fact that $m^{\mathrm{BR}}$ is not $G$-invariant.

1.5. On finiteness of $\mu_{E}^{\mathrm{PS}}$ for geometrically finite $\Gamma$. An important condition for the application of Theorem 1.8 is to determine when $\mu_{E}^{\mathrm{PS}}$ is finite. In this subsection we assume that $\Gamma$ is geometrically finite. Letting $\tilde{E}$ and $\tilde{S}=\pi(\tilde{E})$ be 
as in $\S$ 1.4 suppose further that the natural imbedding $\Gamma_{\tilde{S}} \backslash \tilde{S} \rightarrow \Gamma \backslash \mathbb{H}^{n}$ is proper; in particular, $\mathbf{p}(\tilde{S})$ is closed in $\Gamma \backslash \mathbb{H}^{n}$, where $\Gamma_{\tilde{S}}=\{\gamma \in \Gamma: \gamma \tilde{S}=\tilde{S}\}$.

When $\tilde{S}$ is a point or a horosphere, $\mu_{E}^{\mathrm{PS}}$ is compactly supported (Theorem 4.9).

Theorem 1.11 (Theorem 4.7). If $\tilde{S}$ is totally geodesic, then $\Gamma_{\tilde{S}}$ is geometrically finite.

Definition 1.12 (Parabolic-corank). Let $\Lambda_{\mathrm{p}}(\Gamma)$ denote the set of parabolic fixed points of $\Gamma$ in $\partial \mathbb{H}^{n}$. For any $\xi \in \Lambda_{\mathrm{p}}(\Gamma), \Gamma_{\xi}$ is a virtually free abelian group of rank at least one. Define

$$
\operatorname{pb-corank}\left(\Gamma_{\tilde{S}}\right)=\max _{\xi \in \Lambda_{\mathrm{p}}(\Gamma) \cap \partial(\tilde{S})}\left(\operatorname{rank}\left(\Gamma_{\xi}\right)-\operatorname{rank}\left(\Gamma_{\xi} \cap \Gamma_{\tilde{S}}\right)\right) .
$$

If $\Lambda_{\mathrm{p}}(\Gamma) \cap \partial(\tilde{S})=\emptyset$, we set pb-corank $\left(\Gamma_{\tilde{S}}\right)=0$. In particular, the parabolic corank of $\Gamma_{\tilde{S}}$ is always zero when $\Gamma$ is convex cocompact.

Lemma 1.13 (Lemma 6.2). If $\tilde{S}$ is totally geodesic, then

$$
\operatorname{pb}-\operatorname{corank}\left(\Gamma_{\tilde{S}}\right) \leq \operatorname{codim}(\tilde{S}) .
$$

Theorem 1.14 (Theorems 6.3 and 6.4). We have:

(1) $\operatorname{supp}\left(\mu_{E}^{\mathrm{PS}}\right)$ is compact if and only if $\mathrm{pb}-\operatorname{corank}\left(\Gamma_{\tilde{S}}\right)=0$.

(2) $\left|\mu_{E}^{\mathrm{PS}}\right|<\infty$ if and only if pb-corank $\left(\Gamma_{\tilde{S}}\right)<\delta$.

Note that by [8, Prop. 2], $\delta>\frac{1}{2} \max _{\xi \in \Lambda_{\mathrm{p}}(\Gamma)} \operatorname{rank}\left(\Gamma_{\xi}\right)$. As a consequence of Theorem 1.14, we get:

Corollary 1.15 (Theorem $\underline{6.5}$ ). Suppose that $\operatorname{dim}(\tilde{S}) \geq(n+1) / 2$. If $\left|\mu_{E}^{\mathrm{Leb}}\right|<\infty$, then $\left|\mu_{E}^{\mathrm{PS}}\right|<\infty$.

1.6. Finiteness of $\mu_{E}^{\mathrm{PS}}$ or $\mu_{E}^{\mathrm{Leb}}$ and closedness of $E$. Let $\tilde{E}$ and $E$ be as in 1.4 . In [29], it is shown that $\left|\mu_{E}^{\mathrm{Leb}}\right|<\infty$ implies that $E$ is a closed subset of $\mathrm{T}^{1}\left(\Gamma \backslash \mathbb{H}^{n}\right)$. We prove an analogous statement for $\mu_{E}^{\mathrm{PS}}$.

Theorem 1.16 (Theorem 2.21). Let $\Gamma$ be a discrete Zariski dense subgroup of $G$. If $\left|\mu_{E}^{\mathrm{PS}}\right|<\infty$, then the natural embedding $\Gamma_{\tilde{S}} \backslash \tilde{S} \rightarrow \Gamma \backslash \mathbb{H}^{n}$ is proper.

1.7. Integrability of $\phi_{0}$ and a characterization of a lattice. Define $\phi_{0} \in$ $C\left(\Gamma \backslash \mathbb{H}^{n}\right)$ by

$$
\phi_{0}(x):=\left|\nu_{x}\right| \quad \text { for } x \in \Gamma \backslash \mathbb{H}^{n} .
$$

The function $\phi_{0}$ is an eigenfunction of the hyperbolic Laplace operator with eigenvalue $-\delta(n-1-\delta)$ (see [36]). Sullivan [37] showed that if $\delta>\frac{n-1}{2}$, then $\phi_{0} \in$ $L^{2}\left(\Gamma \backslash \mathbb{H}^{n}, d\right.$ Vol $\left._{\text {Riem }}\right)$ if and only if $\left|m^{\text {BMS }}\right|<\infty$. The following theorem, which is a novel application of Ratner's theorem [30, relates the integrability of $\phi_{0}$ with the finiteness of $\operatorname{Vol}_{\text {Riem }}\left(\Gamma \backslash \mathbb{H}^{n}\right)$ :

Theorem 1.17 (\$3.6). For any discrete subgroup $\Gamma$, the following statements are equivalent:

(1) $\phi_{0} \in L^{1}\left(\Gamma \backslash \mathbb{H}^{n}, d \mathrm{Vol}_{\text {Riem }}\right)$;

(2) $\left|m^{\mathrm{BR}}\right|<\infty$;

(3) $\Gamma$ is a lattice in $G$.

Although $m^{\mathrm{BR}}$ depends on the choice of the base point $o$, its finiteness is independent of the choice. If $\Gamma$ is a lattice, then $\delta=n-1$, and hence $\phi_{0}$ is a constant function by the uniqueness of the harmonic function 38 . 


\section{Transverse measures}

2.1. Let $\left(\mathbb{H}^{n}, d\right)$ denote the hyperbolic $n$-space and $\partial \mathbb{H}^{n}$ its geometric boundary. Let $G$ denote the identity component of the isometry group of $\mathbb{H}^{n}$. We denote by $\mathrm{T}^{1}\left(\mathbb{H}^{n}\right)$ the unit tangent bundle of $\mathbb{H}^{n}$ and by $\pi$ the natural projection from $\mathrm{T}^{1}\left(\mathbb{H}^{n}\right) \rightarrow \mathbb{H}^{n}$. By abuse of notation, we use $d$ to denote a left $G$-invariant metric on $\mathrm{T}^{1}\left(\mathbb{H}^{n}\right)$ such that $d(\pi(u), \pi(v))=\min \left\{d\left(u_{1}, v_{1}\right): \pi\left(u_{1}\right)=\pi(u), \pi\left(v_{1}\right)=\pi(v)\right\}$. For a subset $A$ of $\mathrm{T}^{1}\left(\mathbb{H}^{n}\right)$ or $\mathbb{H}^{n}$ or $\partial \mathbb{H}^{n}$ and a subgroup $H$ of $G$, we denote by $H_{A}$ the stabilizer subgroup $\{g \in H: g(A)=A\}$ of $A$ in $H$.

Denote by $\left\{\mathcal{G}^{r}: r \in \mathbb{R}\right\}$ the geodesic flow on $\mathrm{T}^{1}(\mathbb{H})$. For $u \in \mathrm{T}^{1}\left(\mathbb{H}^{n}\right)$, we set

$$
u^{+}:=\lim _{r \rightarrow \infty} \mathcal{G}^{r}(u) \text { and } u^{-}:=\lim _{r \rightarrow-\infty} \mathcal{G}^{r}(u),
$$

which are the endpoints in $\partial \mathbb{H}^{n}$ of the geodesic defined by $u$. Note that $(g(u))^{ \pm}=$ $g\left(u^{ \pm}\right)$for $g \in G$. The map $\operatorname{Viz}: \mathrm{T}^{1}\left(\mathbb{H}^{n}\right) \rightarrow \partial \mathbb{H}^{n}$ given by $\operatorname{Viz}(u)=u^{+}$is called the visual map.

2.2. The Busemann function $\beta: \partial \mathbb{H}^{n} \times \mathbb{H}^{n} \times \mathbb{H}^{n} \rightarrow \mathbb{R}$ is defined as follows: for $\xi \in \partial \mathbb{H}^{n}$ and $x, y \in \mathbb{H}^{n}$,

$$
\beta_{\xi}(x, y)=\lim _{r \rightarrow \infty} d\left(x, \xi_{r}\right)-d\left(y, \xi_{r}\right)
$$

where $\xi_{r}$ is any geodesic ray tending to $\xi$ as $r \rightarrow \infty$, and the limiting value is independent of the choice of the ray $\xi_{r}$.

Note that $\beta$ is differentiable and invariant under isometries; that is, for $g \in G$ and $x, y \in \mathbb{H}^{n}, \beta_{\xi}(x, y)=\beta_{g(\xi)}(g(x), g(y))$.

For $u \in \mathrm{T}^{1}\left(\mathbb{H}^{n}\right)$, the unstable horosphere based at $u^{-}$is the set

$$
\mathcal{H}_{u}^{+}=\left\{v \in \mathrm{T}^{1}\left(\mathbb{H}^{n}\right): v^{-}=u^{-}, \beta_{u^{-}}(\pi(u), \pi(v))=0\right\},
$$

and the stable horosphere based at $u^{+}$is the set

$$
\mathcal{H}_{u}^{-}=\left\{v \in \mathrm{T}^{1}\left(\mathbb{H}^{n}\right): v^{+}=u^{+}, \beta_{u^{+}}(\pi(u), \pi(v))=0\right\} .
$$

The weak stable manifold corresponding to $u$ is

$$
\begin{gathered}
\tilde{W}_{u}^{s}=\operatorname{Viz}^{-1}\left(u^{+}\right)=\left\{v \in \mathrm{T}^{1}\left(\mathbb{H}^{n}\right): v^{+}=u^{+}\right\}, \\
v_{1}, v_{2} \in \mathcal{H}_{u}^{+}, r \in \mathbb{R} \Rightarrow d\left(\mathcal{G}^{r}\left(v_{1}\right), \mathcal{G}^{r}\left(v_{2}\right)\right)=e^{r} d\left(v_{1}, v_{2}\right), \\
v_{1}, v_{2} \in \tilde{W}_{u}^{s}, r \geq 0 \Rightarrow d\left(\mathcal{G}^{r}\left(v_{1}\right), \mathcal{G}^{r}\left(v_{2}\right)\right) \leq d\left(v_{1}, v_{2}\right) .
\end{gathered}
$$

The image under $\pi$ of a stable or an unstable horosphere $\mathcal{H}$ in $\mathrm{T}^{1}\left(\mathbb{H}^{n}\right)$ based at $\xi$ is called a horosphere in $\mathbb{H}^{n}$ based at $\xi$. Hence $\pi(\mathcal{H})=\left\{y \in \mathbb{H}^{n}: \beta_{\xi}(x, y)=0\right\}$ for $x \in \pi(\mathcal{H})$.

2.3. Let $\tilde{S}$ be one of the following: a horosphere or a complete connected totally geodesic submanifold of $\mathbb{H}^{n}$ of dimension $k$ for $0 \leq k \leq n-1$. Let $\tilde{E} \subset \mathrm{T}^{1}\left(\mathbb{H}^{n}\right)$ denote the unstable horosphere with $\pi(\tilde{E})=\tilde{S}$ if $\tilde{S}$ is a horosphere, and the unit normal bundle over $\tilde{S}$ if $\tilde{S}$ is totally geodesic. 
Lemma 2.1. The visual map Viz restricted to $\tilde{E}$ is a diffeomorphism onto $\partial\left(\mathbb{H}^{n}\right) \backslash$ $\partial(\tilde{S})$.

Proof. The conclusion is obvious if $\tilde{S}$ is a point or a horosphere.

Now suppose that $\tilde{S}$ is a totally geodesic subspace of dimension $1 \leq k \leq n-1$. Consider the upper half space model for $\mathbb{H}^{n}$ :

$$
\mathbb{H}^{n}=\left\{x+j y: x \in \mathbb{R}^{n-1}, y>0, j=(0, \ldots, 0,1)\right\},
$$

and $\partial \mathbb{H}^{n} \cong \mathbb{R}^{n-1} \cup\{\infty\}$. Without loss of generality, we may assume that $\infty \in \partial(\tilde{S})$ and hence $\partial \tilde{S} \backslash\{\infty\}$ is a $(k-1)$-dimensional affine subspace, say $F$, of $\mathbb{R}^{n-1}$. For any $x \in \mathbb{R}^{n-1} \backslash L$, let $x_{1}$ be the orthogonal projection of $x$ on $L$. Let $x_{2}=$ $x_{1}+\left\|x-x_{1}\right\| \cdot j \in \mathbb{H}^{n}$. Let $v \in \mathrm{T}^{1}\left(\mathbb{H}^{n}\right)$ be the unit vector based at $x_{2}$ in the same direction as $x-x_{1}$. Then $v \in \tilde{E}$ and $v^{+}=x$. Now the conclusion of the lemma is straightforward to deduce.

2.3.1. Maps between $\tilde{E}$, and $\mathcal{H}_{v}^{+}$. For $v \in \mathrm{T}^{1}\left(\mathbb{H}^{n}\right),-v$ is the vector with the same base point as $v$ but in the opposite direction. For $v \in \tilde{E}$, let $\xi_{v}: \mathcal{H}_{v}^{+} \backslash \operatorname{Viz}^{-1}(\partial \tilde{S}) \rightarrow$ $\tilde{E} \backslash\{-v\}$ be the map given by

$$
\xi_{v}(u)=\mathrm{Viz}^{-1}\left(u^{+}\right) \cap \tilde{E} .
$$

Then $\xi_{v}$ is a diffeomorphism. Its inverse, $q_{v}: \tilde{E} \backslash\{-v\} \rightarrow \mathcal{H}_{v}^{+} \backslash \operatorname{Viz}^{-1}(\partial \tilde{S})$, is the map given by

$$
q_{v}(w)=\operatorname{Viz}^{-1}\left(w^{+}\right) \cap \mathcal{H}_{v}^{+} .
$$

Proposition 2.2. There exist $C_{1}>0$ and $\epsilon_{0}>0$ such that:

(1) if $v, w \in \tilde{E}$ and $d(v, w)<\epsilon_{0}$, then

$$
\left|\beta_{w^{+}}\left(\pi\left(q_{v}(w)\right), \pi(w)\right)\right| \leq d\left(q_{v}(w), w\right)<C_{1} d(w, v) ;
$$

(2) if $v \in \tilde{E}$ and $w \in \mathcal{H}_{v}^{+}$with $d(v, w)<\epsilon_{0}$, then

$$
\left|\beta_{w^{+}}\left(\pi\left(\xi_{v}(w)\right), \pi(w)\right)\right| \leq d\left(\xi_{v}(w), w\right)<C_{1} d(v, w) .
$$

Proof. In each of the two statements, the first inequality follows directly from the definition of the Busemann function, so we only need to prove the second inequality.

Consider the upper half space model of $\mathbb{H}^{n}$ given by (2.5). By applying an isometry $g \in G$, since $q_{g(v)}(g w)=g\left(q_{v}(w)\right)$, we may assume that $v$ is the unit vector based at $j$ so that $v^{+}=\{\infty\}$.

Since $f(u):=d\left(q_{v}(u), u\right)$ is a differentiable function of $u \in \tilde{E}$, there exist $\epsilon_{0}>0$ and $C_{1}^{\prime}>0$ such that $\|D f(u)\| \leq C_{1}^{\prime}$ for any $u$ with $d(v, u)<\epsilon_{0}$. Therefore, since $f(v)=0$, there exists $C_{1}>0$ such that $|f(u)|=|f(u)-f(v)| \leq C_{1} \cdot d(v, u)$ for all $u \in \tilde{E}$ with $d(u, v)<\epsilon_{0}$. This proves (10), and (2) can be proved similarly.

Remark 2.3. The following stronger form of statements in Proposition 2.2 hold: There exist $\epsilon_{0}>0$ and $C_{1}>0$ such that

$$
\begin{gathered}
\left|\beta_{w^{+}}\left(\pi\left(q_{v}(w)\right), \pi(w)\right)\right| \leq C_{1} d(v, w)^{2}, \text { for all } w \in \tilde{E} \text { with } d(w, v)<\epsilon_{0} \\
\left|\beta_{w^{+}}\left(\pi\left(\xi_{v}(w), \pi(w)\right)\right)\right| \leq C_{1} d(v, w)^{2}, \text { for all } w \in \mathcal{H}_{v}^{+} \text {with } d(w, v)<\epsilon_{0} .
\end{gathered}
$$

We omit a proof, as the stronger version will not be used in this article.

Notation 2.4. Let $\Gamma$ be a non-elementary torsion-free discrete subgroup of $G$ and set $X:=\Gamma \backslash \mathbb{H}^{n}$. Both the natural projection maps $\mathbb{H}^{n} \rightarrow X$ and $\mathrm{T}^{1}\left(\mathbb{H}^{n}\right) \rightarrow \mathrm{T}^{1}(X)$ will be denoted by $\mathbf{p}$. 
2.4. Boxes, plaques and transversals. Let $u \in \mathrm{T}^{1}\left(\mathbb{H}^{n}\right)$. Consider a relatively compact open set $P$ containing $u$ in $\mathcal{H}_{u}^{+}$, and a relatively compact open neighborhood $T$ of $u$ in $\operatorname{Viz}^{-1}\left(u^{+}\right)$. For each $t \in T$ and $p \in P$, the horosphere $\mathcal{H}_{t}^{+}$intersects $\mathrm{Viz}^{-1}\left(p^{+}\right)$at a unique vector: we define

$$
t p:=\mathcal{H}_{t}^{+} \cap \operatorname{Viz}^{-1}\left(p^{+}\right) \in T^{1}\left(\mathbb{H}^{n}\right) .
$$

The map $(t, p) \rightarrow t p$ provides a local chart of a neighborhood of $u$ in $\mathrm{T}^{1}\left(\mathbb{H}^{n}\right)$. Since $u \in P$, in this notation $t u=t$. We call the set

$$
B(u)=\left\{t p \in \mathrm{T}^{1}\left(\mathbb{H}^{n}\right): t \in T, p \in P\right\}
$$

a box around $u$ if some neighborhood of $B(u)$ injects into $\mathrm{T}^{1}(X)$ under $\mathbf{p}$. We write $B=B(u)=T P$.

Note that $P$ (resp. $T$ ) may be disconnected and of 'large' diameter, and then the corresponding $T$ (resp. $P$ ) will be chosen to be of small diameter in order to achieve the required injectivity of $\mathbf{p}$ on a neighborhood of $B(u)$.

For any $t \in T$, the set

$$
t P:=\{t p: p \in P\} \subset \mathcal{H}_{t}^{+}
$$

is called a plaque at $t$, and for any $p \in P$, the set

$$
T p:=\{t p: t \in T\} \subset \mathrm{Viz}^{-1}\left(p^{+}\right)
$$

is called a transversal at $p$. The holonomy map between the transversals $T p$ and $T p^{\prime}$ is given by $t p \mapsto t p^{\prime}$ for all $t \in T$.

Remark 2.5. If $v=t p \in B$, then $t P \subset \mathcal{H}_{v}^{+}, T p \subset \mathrm{Viz}^{-1}\left(v^{+}\right)$and $B(v)=(T p)(t P)$ is a box about $v$ and $T P=(T p)(t P)$. Also, $B(u)$ and $B(v)$ have the same collections of plaques and transversals.

For small $\epsilon>0$, let

$$
\begin{gathered}
T_{\epsilon+}=\left\{s \in \mathrm{Viz}^{-1}\left(u^{+}\right): d(s, T)<\epsilon\right\}, \\
T_{\epsilon-}=\{t \in T: d(t, \partial T)>\epsilon\}, \text { and } B_{\epsilon \pm}=T_{\epsilon \pm} P .
\end{gathered}
$$

Note that for any $\gamma \in G, \gamma P \subset \mathcal{H}_{\gamma u}^{+}, \gamma T \subset \operatorname{Viz}^{-1}\left((\gamma u)^{+}\right), \gamma(t p)=(\gamma t)(\gamma p)$ for any $(t, p) \in T \times P, \gamma(T P)=\gamma(B(u))=B(\gamma u)=(\gamma T)(\gamma P), \gamma(t P)$ is a plaque at $\gamma t$ and $\gamma(T p)$ is a transversal at $\gamma p$. Also, $\gamma B_{\epsilon \pm}=(\gamma T)_{ \pm}(\gamma P)$.

For $r \in \mathbb{R}, \mathcal{G}^{r}(B(u))=B\left(\mathcal{G}^{r}(u)\right)=\left(\mathcal{G}^{r}(T)\right)\left(\mathcal{G}^{r}(P)\right)$.

2.5. For the rest of this section, let $B=T P \subset T^{1}\left(\mathbb{H}^{n}\right)$ denote a box such that $B_{\epsilon_{0}}+$ injects into $\mathrm{T}^{1}(X)$ for some $\epsilon_{0}>0$. By choosing a smaller $\epsilon_{0}$ if necessary, let $C_{1}>0$ be such that Proposition 2.2 holds. Let

$$
C_{2}=\max \left\{d\left(t p_{1}, t p_{2}\right): t \in T_{\epsilon_{0}+}, p_{1}, p_{2} \in P\right\} .
$$

In this section we will develop auxiliary results to understand the intersection of $\mathcal{G}^{r}(E)$ with $\mathbf{p}(B)$ for $r \gg 1$. First we will show that for any $\gamma \in \Gamma$ if $\mathcal{G}^{r}(\gamma \tilde{E}) \cap B$ is non-empty, there exists a unique $t \in T_{\epsilon_{0}} \cap \cap \mathcal{G}^{r}(\gamma \tilde{E})$ and the sets $\mathcal{G}^{r}(\gamma \tilde{E})$ and $\mathcal{G}^{r}(t P)$ are contained in $C_{1} C_{2} e^{-r}$-tubular neighborhoods of each other.

Lemma 2.6. Let $r \in \mathbb{R}$ and $\gamma \in \Gamma$. Suppose that $\mathcal{G}^{r}(\gamma \tilde{E}) \ni$ tp for some $t \in T$, $p \in P$. Let $v=\mathcal{G}^{-r}\left(\gamma^{-1} t p\right) \in \tilde{E}$. Let $p_{1} \in P, y=\mathcal{G}^{-r}\left(\gamma^{-1} t p_{1}\right) \in \mathcal{H}_{v}^{+}$, and $w=\xi_{v}(y) \in \tilde{E}$. Then $w^{+}=y^{+}$,

$$
d(v, y) \leq C_{2} e^{-r} \text { and } d(y, w) \leq C_{1} C_{2} e^{-r} .
$$


Proof. By (2.6), $w^{+}=y^{+}$. Since $t p, t p_{1} \in \mathcal{H}_{t}^{+}$, by (2.3) and (2.8),

$$
\begin{aligned}
d(v, y)=d\left(\mathcal{G}^{-r}\left(\gamma^{-1} t p\right), \mathcal{G}^{-r}\left(\gamma^{-1} t p_{1}\right)\right) & =d\left(\mathcal{G}^{-r}(t p), \mathcal{G}^{-r}\left(t p_{1}\right)\right) \\
& \leq d\left(t p, t p_{1}\right) e^{-r} \leq C_{2} e^{-r} .
\end{aligned}
$$

By Proposition 2.2, $d(y, w)=d\left(y, \xi_{v}(y)\right) \leq C_{1} d(v, y) \leq C_{1} C_{2} e^{-r}$.

Lemma 2.7. For any $r \in \mathbb{R}$ and $\gamma \in \Gamma$,

$$
\#\left(T \cap \mathcal{G}^{r}(\gamma \tilde{E})\right)=\#\left(\mathcal{G}^{-r}\left(\gamma^{-1} T\right) \cap \tilde{E}\right) \leq 1 .
$$

Proof. Since $\operatorname{Viz}\left(\mathcal{G}^{-r}\left(\gamma^{-1} T\right)\right)=\gamma^{-1} \operatorname{Viz}(T)$ is a singleton set and $\operatorname{Viz}$ restricted to $\tilde{E}$ is injective, the conclusion follows.

Notation 2.8. For $r \in \mathbb{R}$ and $\gamma \in \Gamma$, in view of Lemma 2.7 define

$$
\tilde{E}_{r, \gamma}= \begin{cases}\xi_{\mathcal{G}^{-r}\left(\gamma^{-1} t\right)}\left(\mathcal{G}^{-r}\left(\gamma^{-1} t P\right)\right) \subset \tilde{E} & \text { if } T \cap \mathcal{G}^{r}(\gamma \tilde{E})=\{t\} \\ \emptyset & \text { if } T \cap \mathcal{G}^{r}(\gamma \tilde{E})=\emptyset .\end{cases}
$$

Proposition 2.9. For any $0<\epsilon \leq \epsilon_{0}, r>r_{\epsilon}:=\log \left(C_{1}\left(C_{1}+1\right) C_{2} / \epsilon\right)$ and $\gamma \in \Gamma$, we have

$$
\mathcal{G}^{-r}\left(\gamma^{-1} B_{\epsilon-}\right) \cap \tilde{E} \subset \tilde{E}_{r, \gamma} \subset \mathcal{G}^{-r}\left(\gamma^{-1} B_{\epsilon+}\right) \cap \tilde{E}
$$

Proof of first inclusion in (2.10). Let $\gamma \in \Gamma, t \in T_{\epsilon-}$ and $p \in P$ be such that $v:=\mathcal{G}^{-r}\left(\gamma^{-1} t p\right) \in \tilde{E}$. Let $y=\mathcal{G}^{-r}\left(\gamma^{-1} t\right)$ and $w=\xi_{v}(y) \in \tilde{E}$. By Lemma 2.6.

$$
d(y, w) \leq C_{1} C_{2} e^{-r}<\epsilon /\left(C_{1}+1\right)<\epsilon .
$$

Let $t_{1}=\mathcal{G}^{r}(\gamma w)$. Since $t=\mathcal{G}^{r}(\gamma y)$ and $w^{+}=y^{+}, t_{1}^{+}=t^{+}$. By (2.4),

$$
d\left(t, t_{1}\right)=d\left(\mathcal{G}^{r}(\gamma y), \mathcal{G}^{r}(\gamma w)\right) \leq d(\gamma y, \gamma w)=d(y, w)<\epsilon .
$$

Therefore $t_{1} \in T$, for $t \in T_{\epsilon-}$. Since $\left(t_{1} p\right)^{+}=(t p)^{+}$, we have

$$
\mathcal{G}^{-r}\left(\gamma^{-1} t_{1} p\right)^{+}=\mathcal{G}^{-r}\left(\gamma^{-1} t p\right)^{+}=v^{+} .
$$

Since $w=\mathcal{G}^{-r}\left(\gamma^{-1} t_{1}\right), \mathcal{G}^{-r}\left(\gamma^{-1} t_{1} p\right) \in \mathcal{H}_{w}^{+}$. Also, $w, v \in \tilde{E}$. Therefore by (2.6),

$$
v=\xi_{w}\left(\mathcal{G}^{-r}\left(\gamma^{-1} t_{1} p\right)\right) \in \tilde{E}_{r, \gamma} .
$$

Proof of second inclusion in (2.10). By Lemma 2.7 let $\{t\}=T \cap \mathcal{G}^{r}(\gamma \tilde{E})$ for some $\gamma \in \Gamma$. Let $v=\mathcal{G}^{-r}\left(\gamma^{-1} t\right) \in \tilde{E}, p \in P, y=\mathcal{G}^{-r}\left(\gamma^{-1} t p\right)$, and $w=\xi_{v}(y) \in \tilde{E}_{r, \gamma}$. By Lemma 2.6.

$$
d(v, w) \leq d(v, y)+d(y, w) \leq C_{2} e^{-r}+C_{1} C_{2} e^{-r} \leq \epsilon / C_{1} .
$$

Put $v_{1}=q_{w}(v) \in \mathcal{H}_{w}^{+}$. By (2.7), $v_{1}^{+}=v^{+}$, and by Proposition 2.2(1),

$$
d\left(v, v_{1}\right) \leq C_{1} d(v, w) \leq \epsilon .
$$

Put $t_{1}=\mathcal{G}^{r}\left(\gamma v_{1}\right) \in \mathcal{H}_{\mathcal{G}^{r}(\gamma w)}^{+}$. Since $t=\mathcal{G}^{r}(\gamma v), t_{1}^{+}=t^{+}$. By (2.4),

$$
d\left(t, t_{1}\right)=d\left(\mathcal{G}^{r}(\gamma v), \mathcal{G}^{r}\left(\gamma v_{1}\right)\right) \leq d\left(\gamma v, \gamma v_{1}\right) \leq \epsilon .
$$

Hence $t_{1} \in T_{\epsilon+}$. Now $\mathcal{G}^{r}(\gamma w), t_{1} p \in \mathcal{H}_{t_{1}}^{+}$. Since $w^{+}=y^{+}$,

$$
\left(\mathcal{G}^{r}(\gamma w)\right)^{+}=\left(\mathcal{G}^{r}(\gamma y)\right)^{+}=(t p)^{+}=\left(t_{1} p\right)^{+} .
$$

Since Viz is injective on $\mathcal{H}_{t_{1}}^{+}, \mathcal{G}^{r}(\gamma w)=t_{1} p$. Hence $w \in \mathcal{G}^{-r}\left(\gamma^{-1} B_{\epsilon+}\right)$. 
2.6. Measure on $E$ corresponding to a conformal density on $\partial \mathbb{H}^{n}$. Let $\left\{\mu_{x}: x \in \mathbb{H}^{n}\right\}$ be a $\Gamma$-invariant conformal density of dimension $\delta_{\mu}>0$ on $\partial \mathbb{H}^{n}$. That is, for each $x \in \mathbb{H}^{n}, \mu_{x}$ is a positive finite Borel measure on $\partial \mathbb{H}^{n}$ such that for all $y \in \mathbb{H}^{n}, \xi \in \partial \mathbb{H}^{n}$ and $\gamma \in \Gamma$,

$$
\gamma_{*} \mu_{x}=\mu_{\gamma x} \quad \text { and } \quad \frac{d \mu_{x}}{d \mu_{y}}(\xi)=e^{\delta_{\mu} \beta_{\xi}(y, x)},
$$

where $\gamma_{*} \mu_{x}(F):=\mu_{x}\left(\gamma^{-1}(F)\right)$ for any Borel subset $F$ of $\partial \mathbb{H}^{n}$.

Fix $o \in \mathbb{H}^{n}$. We consider the measure on $\tilde{E}$ given by

$$
d \mu_{\tilde{E}}(v)=e^{\delta_{\mu} \beta_{v+}(o, \pi(v))} d \mu_{o}\left(v^{+}\right) .
$$

By (2.11), $\mu_{\tilde{E}}$ is independent of the choice of $o \in \mathbb{H}^{n}$ and $\gamma_{*} \mu_{\tilde{E}}=\mu_{\gamma \tilde{E}}$ for any $\gamma \in \Gamma$. Let $\mu_{\Gamma_{\tilde{E}} \backslash \tilde{E}}$ be the locally finite Borel measure on $\Gamma_{\tilde{E}} \backslash \tilde{E}$ induced by $\mu_{\tilde{E}}$ as follows: For any $f \in C_{c}(\tilde{E})$, let $\bar{f}\left(\Gamma_{\tilde{E}} v\right)=\sum_{\gamma \in \Gamma} f(\gamma v)$, for all $v \in \tilde{E}$. Then $f \mapsto \bar{f}$ is a surjective map from $C_{c}(\tilde{E})$ from to $C_{c}\left(\Gamma_{\tilde{E}} \backslash \tilde{E}\right)$, and

$$
\int_{\Gamma_{\tilde{E} \backslash \tilde{E}}} \bar{f} d \mu_{\Gamma_{\tilde{E}} \backslash \tilde{E}}:=\int_{\tilde{E}} f d \mu_{\tilde{E}}
$$

is well defined; see [29, Chapter 1] for a similar construction.

Now let $\mu_{E}$ be the measure on $E=\mathbf{p}(\tilde{E})$ defined as the pushforward of $\mu_{\Gamma_{\tilde{E}} \backslash \tilde{E}}$ from $\Gamma_{\tilde{E}} \backslash \tilde{E}$ to $T^{1}\left(\Gamma \backslash \mathbb{H}^{n}\right)$ under the map $\Gamma_{\tilde{E}} v \mapsto \Gamma v$. Thus for any set $B \subset \mathrm{T}^{1}\left(\mathbb{H}^{n}\right)$ such that $\mathbf{p}$ is injective on $B$ and for any measurable non-negative function $f$ on $E \cap \mathbf{p}(B)$,

$$
\begin{aligned}
\int_{E \cap \mathbf{p}(B)} f d \mu_{E} & =\sum_{[\gamma] \in \Gamma / \Gamma_{\tilde{E}}} \int_{u \in \gamma \tilde{E} \cap B} f(\mathbf{p}(u)) d \mu_{\gamma \tilde{E}}(u) \\
& =\sum_{[\gamma] \in \Gamma / \Gamma_{\tilde{E}}} \int_{u \in \tilde{E} \cap \gamma^{-1} B} f(\mathbf{p}(u)) d \mu_{\tilde{E}}(u),
\end{aligned}
$$

where the integration over an empty set is defined to be 0 . Therefore by Proposition 2.9 we obtain the following:

Proposition 2.10. Let $0<\epsilon \leq \epsilon_{0}$ and $r>r_{\epsilon}$. Then for all Borel measurable functions $\Psi \geq 0$ on $\mathrm{T}^{1}(X)$ with $\operatorname{supp}(\Psi) \subset \mathbf{p}\left(B_{\epsilon-}\right)$ and $f \geq 0$ on $E$, we have

$$
\begin{aligned}
& \int_{u \in E} \Psi\left(\mathcal{G}^{r}(u)\right) f(u) d \mu_{E}(u)=\int_{E \cap \mathbf{p}\left(\mathcal{G}^{-r}\left(B_{\epsilon \pm}\right)\right)} \Psi\left(\mathcal{G}^{r}(u)\right) f(u) d \mu_{E}(u) \\
& =\sum_{[\gamma] \in \Gamma / \Gamma_{\tilde{E}}} \int_{\mathcal{G}^{-r}\left(\gamma^{-1} B_{\epsilon \pm}\right) \cap \tilde{E}} \Psi\left(\mathcal{G}^{r}(u)\right) f(u) d \mu_{\tilde{E}}(u) \\
& =\sum_{[\gamma] \in \Gamma / \Gamma_{\tilde{E}}} \int_{\tilde{E}_{r, \gamma}} \Psi\left(\mathcal{G}^{r}(\mathbf{p}(u))\right) f(\mathbf{p}(u)) d \mu_{\tilde{E}}(u) \text {. }
\end{aligned}
$$

Remark 2.11. (1) For the counting application in \$7, we will use the results of this section only for the case when the map $\Gamma_{\tilde{E}} \backslash \tilde{E} \rightarrow \mathrm{T}^{1}\left(\Gamma \backslash \mathbb{H}^{n}\right)$ is proper, in which case $\mu_{E}$ is a locally finite Borel measure.

(2) In the general case, $\mu_{E}$ may not be $\sigma$-finite, but it is an $s$-finite measure; namely, a countable sum of finite measures (with possibly non-disjoint supports).

(3) If the dimension of $\tilde{S}=\pi(\tilde{E})$ in $\mathbb{H}^{n}$ is 0 or $n-1$, the map $\Gamma_{\tilde{E}} \backslash \tilde{E}$ to $\mathrm{T}^{1}\left(\Gamma \backslash \mathbb{H}^{n}\right)$ is injective, and hence $\mu_{E}$ is $\sigma$-finite on $\mathrm{T}^{1}\left(\Gamma \backslash \mathbb{H}^{n}\right)$.

2.6.1. Measures on horospherical foliation and their semi-invariance under geodesic flow. The conformal density $\left\{\mu_{x}\right\}$ induces a $\Gamma$-equivariant family of measures $\left\{\mu_{\mathcal{H}_{u}^{+}}: u \in \mathrm{T}^{1}\left(\mathbb{H}^{n}\right)\right\}$ on the unstable horospherical foliation on $\mathrm{T}^{1}\left(\mathbb{H}^{n}\right)$ :

$$
d \mu_{\mathcal{H}_{u}^{+}}(v)=e^{\delta_{\mu} \beta_{v+}(o, \pi(v))} d \mu_{o}\left(v^{+}\right) .
$$


For any $r \in \mathbb{R}$, since $\mathcal{G}^{r}(v)^{+}=v^{+}$and

$$
\beta_{v^{+}}\left(o, \pi\left(\mathcal{G}^{r}(v)\right)\right)-\beta_{v^{+}}(o, \pi(v))=\beta_{v^{+}}\left(\pi(v), \pi\left(\mathcal{G}^{r}(v)\right)\right)=r,
$$

by (2.11), we get for all $\gamma \in \Gamma$ and $r \in \mathbb{R}$,

$$
\gamma_{*} \mu_{\mathcal{H}_{u}^{+}}=\mu_{\mathcal{H}_{\gamma u}^{+}} \quad \text { and } \quad \mathcal{G}_{*}^{r} \mu_{\mathcal{H}_{u}^{+}}=e^{-\delta_{\mu} r} \mu_{\mathcal{H}_{\mathcal{G}^{r}(u)}^{+}} \text {. }
$$

2.7. On transversal intersections of, $\mathcal{G}^{r}(\Gamma \tilde{E})$ with $B$. Let a box $B, \epsilon_{0}>0$, $C_{1}>0$ and $C_{2}>0$ be as described in the beginning of 2.5 . For any $0<\epsilon \leq \epsilon_{0}$, we put

$$
r_{\epsilon}=\log \left(\left(C_{1}+1\right) C_{1} C_{2} / \epsilon\right) .
$$

Proposition 2.12. Let $0<\epsilon \leq \epsilon_{0}, r>r_{\epsilon}$, and $\{t\}=T \cap \mathcal{G}^{r}(\gamma \tilde{E})$ for some $\gamma \in \Gamma$. Then for all measurable functions $\Psi \geq 0$ on $B_{\epsilon_{0}}$ and $f \geq 0$ on $\tilde{E}$,

$$
\begin{aligned}
& \left(e^{-\delta_{\mu} \epsilon}\right) f_{\epsilon}^{-}\left(\mathcal{G}^{-r}\left(\gamma^{-1} t\right)\right) \int_{t P} \Psi_{\epsilon}^{-} d \mu_{\mathcal{H}_{t}^{+}} \\
\leq & e^{\delta_{\mu} r} \int_{w \in \tilde{E}_{r, \gamma}} \Psi\left(\mathcal{G}^{r}(\gamma w)\right) f(w) d \mu_{\tilde{E}}(w) \\
\leq & \left(e^{\delta_{\mu} \epsilon}\right) f_{\epsilon}^{+}\left(\mathcal{G}^{-r}\left(\gamma^{-1} t\right)\right) \int_{t P} \Psi_{\epsilon}^{+} d \mu_{\mathcal{H}_{t}^{+}},
\end{aligned}
$$

where $f_{\epsilon}^{ \pm}$on $\tilde{E}$ and $\Psi_{\epsilon}^{ \pm}$on $B_{\epsilon+}$ are defined as

$$
\begin{aligned}
& f_{\epsilon}^{+}(u)=\sup _{\left\{u_{1} \in \tilde{E}: d\left(u_{1}, u\right) \leq \epsilon\right\}} f\left(u_{1}\right), \\
& f_{\epsilon}^{-}(u)=\inf _{\left\{u_{1} \in \tilde{E}: d\left(u_{1}, u\right) \leq \epsilon\right\}} f\left(u_{1}\right), \\
& \Psi_{\epsilon}^{+}(t p)=\sup _{\left\{t_{1} \in T_{\epsilon+}: d\left(t_{1} p, t p\right) \leq \epsilon\right\}} \Psi\left(t_{1} p\right), \\
& \Psi_{\epsilon}^{-}(t p)=\inf _{\left\{t_{1} \in T_{\epsilon+}: d\left(t_{1} p, t p\right) \leq \epsilon\right\}} \Psi\left(t_{1} p\right) .
\end{aligned}
$$

Proof. Let $v=\mathcal{G}^{-r}\left(\gamma^{-1} t\right) \in \tilde{E}$. Let $\phi: t P \subset \mathcal{H}_{t}^{+} \rightarrow \tilde{E}_{r, \gamma} \subset \tilde{E}$ be the map given by $\phi(t p)=w:=\xi_{v}(y)$, where $p \in P$ and $y=\mathcal{G}^{-r}\left(\gamma^{-1} t p\right)$. By Lemma 2.6.

$$
d(y, w)<C_{1} C_{2} e^{-r}<\epsilon, \quad d(v, w)<\left(C_{1}+1\right) C_{2} e^{-r}<\epsilon,
$$

and since $w^{+}=y^{+}$,

$$
d\left(\mathcal{G}^{r}(\gamma y), \mathcal{G}^{r}(\gamma w)\right)=d\left(\mathcal{G}^{r}(y), \mathcal{G}^{r}(w)\right) \leq d(y, w)<\epsilon,
$$

and by Proposition 2.9, $\mathcal{G}^{r}(\gamma w) \in T_{\epsilon+} p$. Therefore,

$$
\begin{gathered}
f_{\epsilon}^{-}(v) \leq f(w) \leq f_{\epsilon}^{+}(v), \\
\Psi_{\epsilon}^{-}\left(\mathcal{G}^{r}(\gamma y)\right) \leq \Psi\left(\mathcal{G}^{r}(\gamma w)\right) \leq \Psi_{\epsilon}^{+}\left(\mathcal{G}^{r}(\gamma y)\right) .
\end{gathered}
$$

For the map $t p \mapsto y:=\mathcal{G}^{-r}\left(\gamma^{-1} t p\right)$, by (2.16),

$$
e^{\delta_{\mu} r} d \mu_{\mathcal{H}_{v}^{+}}(y)=d \mu_{\mathcal{H}_{t}^{+}}(t p) .
$$

For the map $y \mapsto w=\xi_{v}(y)$, by (2.12) and (2.15), since $w^{+}=y^{+}$,

$$
d \mu_{\tilde{E}}(w)=\frac{e^{\delta_{\mu} \beta_{w^{+}}(o, \pi(w))}}{e^{\delta_{\mu} \beta_{y^{+}}(o, \pi(y))}} d \mu_{\mathcal{H}_{v}^{+}}(y)=e^{\delta_{\mu} \beta_{w^{+}}(\pi(y), \pi(w))} d \mu_{\mathcal{H}_{v}^{+}}(y) .
$$

By (2.19),$\left|\beta_{w^{+}}(\pi(y), \pi(w))\right| \leq d(\pi(y), \pi(w)) \leq \epsilon$. Therefore,

$$
e^{-\delta_{\mu} \epsilon}<d \mu_{\tilde{E}}(w) / d \mu_{\mathcal{H}_{v}^{+}}(y)<e^{\delta_{\mu} \epsilon} .
$$


Combining (2.22) and (2.24), for the map $w=\phi(t p)$ we get

$$
e^{-\delta_{\mu} \epsilon} \leq e^{\delta_{\mu} r} \frac{d \mu_{\tilde{E}}(w)}{d \mu_{\mathcal{H}_{t}^{+}}(t p)} \leq e^{\delta_{\mu} \epsilon}
$$

By noting that $\mathcal{G}^{-r}\left(\gamma^{-1} t\right)=v$ and $t p=\mathcal{G}^{r}(\gamma y)$, the conclusion of the proposition follows from (2.20), (2.21) and (2.25).

Notation 2.13. For $r \geq 0$ and $t \in T \cap \mathcal{G}^{r}(\Gamma \tilde{E})$, in view of Lemma 2.7 let

$$
\bar{\Gamma}_{r, t}=\left\{[\gamma] \in \Gamma / \Gamma_{\tilde{E}}:\{t\}=T \cap \mathcal{G}^{r}(\gamma \tilde{E})\right\} .
$$

Since $\mathbf{p}$ is injective on $B_{\epsilon_{0}+}$, for notational convenience we identify $t \in T_{\epsilon_{0}+}$ with its image $\mathbf{p}(t) \in \mathbf{p}(T) \subset X$. Therefore we have

$$
\left\{[\gamma] \in \Gamma / \Gamma_{\tilde{E}}: \tilde{E}_{r, \gamma} \neq \emptyset\right\}=\bigcup_{t \in T \cap \mathcal{G}^{r}(\Gamma \tilde{E})} \bar{\Gamma}_{r, t}=\bigcup_{t \in T \cap \mathcal{G}^{r}(E)} \bar{\Gamma}_{r, t} .
$$

Combining Proposition 2.10 and Proposition 2.12, in view of 2.27) we deduce the following:

Corollary 2.14. Let $0<\epsilon \leq \epsilon_{0}$ and $r>r_{\epsilon}$. For all measurable functions $\Psi \geq 0$ on $B_{\epsilon_{0}}+$ with $\operatorname{supp}(\Psi) \subset B_{\epsilon-}$ and $f \geq 0$ on $E$, we have

$$
\begin{aligned}
& \left(e^{-\delta_{\mu} \epsilon}\right) \sum_{t \in T \cap \mathcal{G}^{r}(E)} \#\left(\bar{\Gamma}_{r, t}\right) f_{\epsilon}^{-}\left(\mathcal{G}^{-r}(t)\right) \cdot \int_{t P} \Psi_{\epsilon}^{-} d \mu_{\mathcal{H}_{t}^{+}} \\
\leq & e^{\delta_{\mu} r} \int_{E} \Psi\left(\mathcal{G}^{r}(u)\right) f(u) d \mu_{E}(u) \\
\leq & \left(e^{\delta_{\mu} \epsilon}\right) \sum_{t \in T \cap \mathcal{G}^{r}(E)} \#\left(\bar{\Gamma}_{r, t}\right) \cdot f_{\epsilon}^{+}\left(\mathcal{G}^{-r}(t)\right) \cdot \int_{t P} \Psi_{\epsilon}^{+} d \mu_{\mathcal{H}_{t}^{+}},
\end{aligned}
$$

where $f_{\epsilon}^{ \pm}$on $B_{\epsilon+}$ and $\Psi_{\epsilon}^{ \pm}$on $E$ are defined as in (2.18).

\subsection{Haar system and admissible boxes.}

Lemma 2.15 (31]). For a uniformly continuous $\Psi \in C(B)$, the map

$$
t \in T \mapsto \int_{t P} \Psi d \mu_{\mathcal{H}_{t}^{+}}
$$

is uniformly continuous. In particular, the map $t \mapsto \mu_{\mathcal{H}_{t}^{+}}(t P)$ is uniformly continuous.

Proof. Note that $(t p)^{+}=p^{+}$. Therefore by (2.15)

$$
\int_{t P} \Psi d \mu_{\mathcal{H}_{t}^{+}}=\int_{P} \Psi(t p) e^{\delta_{\mu} \beta_{p^{+}}(o, \pi(t p))} d \mu_{o}\left(p^{+}\right) .
$$

Put $\phi(t p)=\Psi(t p) e^{\delta_{\mu} \beta_{p^{+}}(o, \pi(t p))}$. Since $\phi$ is uniformly continuous on $B$,

$$
\left|\int_{t_{1} P} \Psi d \mu_{\mathcal{H}_{t_{1}}^{+}}-\int_{t_{2} P} \Psi d \mu_{\mathcal{H}_{t_{2}}^{+}}\right| \leq \mu_{o}(\operatorname{Viz}(P)) \cdot \sup _{p \in P}\left|\phi\left(t_{1} p\right)-\phi\left(t_{2} p\right)\right| \rightarrow 0
$$

as $d\left(t_{1}, t_{2}\right) \rightarrow 0$. 
Definition 2.16. A box $B=T P$ as defined in $\$ 2.4$ is called admissible with respect to the conformal density $\left\{\mu_{x}\right\}$ if every plaque of $B$ has a positive measure with respect to $\left\{\mu_{\mathcal{H}^{+}}\right\}$; that is, $\mu_{\mathcal{H}_{t}^{+}}(t P)>0$ for all $t \in T$ or, equivalently,

$$
\mu_{x}(\operatorname{Viz}(t P))=\mu_{x}\left(P^{+}\right)>0 \text { for some (and hence all) } x \in \mathbb{H}^{n} \text {. }
$$

Lemma 2.17. Fix a conformal density $\left\{\mu_{x}\right\}_{x \in \mathbb{H}^{n}}$ on $\partial \mathbb{H}^{n}$. Then for any $u \in$ $\mathrm{T}^{1}\left(\mathbb{H}^{n}\right)$, there exists an admissible box around $u$ with respect to $\left\{\mu_{x}\right\}$.

Proof. Fix any $x \in \mathbb{H}^{n}$. Since $\Gamma_{u^{-}}$is virtually abelian and since we assume that $\Gamma$ is non-elementary, $\Gamma$ does not fix $u^{-}$. Therefore by the $\Gamma$-invariance and the conformality of the density $\left\{\mu_{x}\right\}$, we have $\operatorname{supp}\left(\mu_{x}\right) \neq\left\{u^{-}\right\}$. Since Viz : $\mathcal{H}_{u}^{+} \rightarrow$ $\partial \mathbb{H}^{n} \backslash\left\{u^{-}\right\}$is a diffeomorphism, there exists $u_{1} \in \mathcal{H}_{u}^{+}$such that $u_{1}^{+}=\operatorname{Viz}\left(u_{1}\right) \in$ $\operatorname{supp}\left(\mu_{x}\right)$. If $\gamma u=u_{1}$ for any $\gamma \in \Gamma$, then by the conformality, $u \in \operatorname{supp}\left(\mu_{\mathcal{H}_{u}^{+}}\right)$and we replace $u_{1}$ by $u$. Since $\mathbf{p}$ is injective on $\left\{u, u_{1}\right\}$, there exists a relatively compact open subset $P$ of $\mathcal{H}_{u}^{+}$containing $\left\{u, u_{1}\right\}$ such that $\mathbf{p}$ is injective on an open set $\Omega$ of $\mathrm{T}^{1}\left(\mathbb{H}^{n}\right)$ containing $\bar{P}$. Then $\mu_{x}(\operatorname{Viz}(P))>0$. By Lemma 2.15, we can choose $T$ a large enough ball in $\mathrm{Viz}^{-1}\left(u^{+}\right)$so that some neighborhood of the closure of $B=T P$ is contained in $\Omega$. Now $B=T P$ is an admissible box.

2.8.1. Let $B=T P$ be an admissible box with respect to a conformal density $\left\{\mu_{x}\right\}$ such that $\mathbf{p}$ is injective on a neighborhood of the closure of $B_{\epsilon_{0}}+$ for some $\epsilon_{0}>0$. Let $C_{1}, C_{2}$ be as described at the beginning of $\$ 2.5$. For notational convenience, we will identify $T_{\epsilon_{0}}$ and $B_{\epsilon_{0}}+$ with their respective images in $\mathrm{T}^{1}(X)$ under $\mathbf{p}$.

Proposition 2.18. Let $0<\epsilon \leq \epsilon_{0}$ and $r>r_{\epsilon}$ (see (2.17)). Then for all measurable functions $\psi \geq 0$ on $T_{\epsilon_{0+}}$ with $\operatorname{supp}(\psi) \subset T_{\epsilon-}$ and $f \geq 0$ on $E$, we have

$$
\begin{aligned}
& \left(e^{-\delta_{\mu} \epsilon}\right) \int_{E} \Psi_{\epsilon}^{-}\left(\mathcal{G}^{r}(w)\right) f_{\epsilon}^{-}(w) d \mu_{E}(w) \\
\leq & e^{-\delta_{\mu} r} \sum_{t \in T \cap \mathcal{G}^{r}(E)} \#\left(\bar{\Gamma}_{r, t}\right) \cdot \psi(t) f\left(\mathcal{G}^{-r}(t)\right) \\
\leq & \left(e^{-\delta_{\mu} \epsilon}\right) \int_{E} \Psi_{\epsilon}^{+}\left(\mathcal{G}^{r}(w)\right) f_{\epsilon}^{+}(w) d \mu_{E}(w),
\end{aligned}
$$

where the function $\Psi$ on $B_{\epsilon_{0}}+$ is defined by

$$
\Psi(\mathbf{p}(t p)):=\psi(t) / \mu_{\mathcal{H}_{t}^{+}}(t P), \text { for all }(t, p) \in T_{\epsilon_{0}+} \times P,
$$

and $\Psi_{\epsilon}^{ \pm}$on $B_{\epsilon+}$ and $f_{\epsilon}^{ \pm}$on $E$ are defined as in (2.18).

Proof. Since $\int_{t P} \Psi d \mu_{\mathcal{H}_{t}^{+}}=\psi(t)$, the result is straightforward to deduce from Corollary 2.14 .

In 93 Proposition 2.18 will enable us to describe the limiting distribution of the transversal intersections $T \cap \mathcal{G}^{r}(E)$ using the mixing of the geodesic flow with respect to $m^{\mathrm{BMS}}$ (cf. Theorem 3.5).

2.9. Some direct consequences. The results proved in this subsection are also of independent interest. Let the notation be as in 2.8 .1

Corollary 2.19. Let $0<\epsilon \leq \epsilon_{0}$ and $f$ be a measurable function on $E$ such that $f_{\epsilon}^{+} \in L^{1}\left(E, \mu_{E}\right)$. Then for any $r>r_{\epsilon}$ and any measurable function $\psi$ on $T$,

$$
\sum_{t \in T \cap \mathcal{G}^{r}(E)} \#\left(\bar{\Gamma}_{r, t}\right) \cdot\left|\psi(t) f\left(\mathcal{G}^{-r}(t)\right)\right|<\infty .
$$


In particular, if there exists a $\Gamma$-invariant conformal density $\left\{\mu_{x}\right\}$ and $\left|\mu_{E}\right|<\infty$, then

$$
\sum_{t \in T \cap \mathcal{G}^{r} E} \#\left(\bar{\Gamma}_{r, t}\right)<\infty
$$

Proof. By Proposition 2.18 with $T_{\epsilon}$ in place of $T$ and declaring $\psi$ to be zero outside $T$, we obtain the first claim because

$$
\sum_{t \in T \cap \mathcal{G}^{r}(E)} \#\left(\bar{\Gamma}_{r, t}\right) \cdot\left|\psi(t) f\left(\mathcal{G}^{-r}(t)\right)\right| \leq(1+\epsilon) e^{\delta_{\mu} r}\left\|\Psi_{\epsilon}^{+}\right\|_{\infty} \cdot \mu_{E}\left(\left|f_{\epsilon}^{+}\right|\right) .
$$

To deduce the second claim from the first one, we choose $f=1$ on $E$ and $\psi=1$ on $T$.

Definition 2.20 (Radial limit points). The limit set $\Lambda(\Gamma)$ of $\Gamma$ is the set of all accumulation points of an orbit $\Gamma(z)$ in $\overline{\mathbb{H}}^{n}$ for $z \in \mathbb{H}^{n}$. As $\Gamma$ acts properly discontinuously on $\mathbb{H}^{n}, \Lambda(\Gamma)$ is contained in $\partial \mathbb{H}^{n}$.

A point $\xi \in \Lambda(\Gamma)$ is called a radial limit point if for some (and hence every) geodesic ray $\beta$ tending to $\xi$ and some (and hence every) point $x \in \mathbb{H}^{n}$, there is a sequence $\gamma_{i} \in \Gamma$ with $\gamma_{i} x \rightarrow \xi$, and $d\left(\gamma_{i} x, \beta\right)$ is bounded.

We denote by $\Lambda_{\mathrm{r}}(\Gamma)$ the set of radial limit points for $\Gamma$.

If $\Gamma$ is non-elementary, $\Lambda_{\mathrm{r}}(\Gamma)$ is a non-empty $\Gamma$-invariant subset of $\Lambda(\Gamma)$. Since $\Lambda(\Gamma)$ is a $\Gamma$-minimal closed subset of $\partial \mathbb{H}^{n}$, we have that $\overline{\Lambda_{\mathrm{r}}(\Gamma)}=\Lambda(\Gamma)$.

Theorem 2.21. Let $C$ denote the smallest subsphere of $\mathbb{H}^{n}$ containing $\Lambda(\Gamma)$. Suppose that $C=\partial \tilde{S}$ or $\operatorname{dim}(C)>\operatorname{dim}(\partial \tilde{S})$. If there exists a $\Gamma$-invariant conformal density $\left\{\mu_{x}: x \in \mathbb{H}^{n}\right\}$ such that $\left|\mu_{E}\right|<\infty$, then the natural map $\overline{\mathbf{p}}: \Gamma_{\tilde{E}} \backslash \tilde{E} \rightarrow$ $\Gamma \backslash \mathrm{T}^{1}\left(\mathbb{H}^{n}\right)$ is proper.

Proof. Note that $\Gamma \subset G_{C}=\{g \in G: g C=C\}$, because if $\gamma \in \Gamma$, then $\gamma C \cap C \supset$ $\Lambda(\Gamma)$, hence by minimality $\gamma C=C$.

Suppose $C=\partial \tilde{S}$. Then, since $G_{\tilde{S}}=G_{\partial \tilde{S}}, \Gamma=\Gamma \cap G_{C}=\Gamma_{\tilde{S}}=\Gamma_{\tilde{E}}$, and hence the properness of $\overline{\mathbf{p}}$ is obvious.

Now suppose that $\operatorname{dim}(C)>\operatorname{dim}(\tilde{S})$ and that that $\overline{\mathbf{p}}$ is not proper. Then there exist sequences $\gamma_{i} \in \Gamma$ and $e_{i} \in \tilde{E}$ such that $\gamma_{i} e_{i}$ converges to a vector $v \in \mathrm{T}^{1}\left(\mathbb{H}^{n}\right)$ as $i \rightarrow \infty$, and

$$
\gamma_{i} \Gamma_{\tilde{E}} \neq \gamma_{j} \Gamma_{\tilde{E}}, \text { for all } i \neq j .
$$

Fix $e_{0} \in \tilde{E}$. Since $G_{\tilde{E}}$ acts transitively on $\tilde{E}$, there exists $h_{i} \in G_{\tilde{E}}$ such that $e_{i}=h_{i} e_{0}$. Then $\gamma_{i} h_{i} e_{0}$ converges to $v$. Therefore there exists $g \in G$ such that $\gamma_{i} h_{i} \rightarrow g$ and $v=g e_{0}$.

Now $\operatorname{Viz}(g \tilde{E})=\partial \mathbb{H}^{n}-\partial(g \tilde{S})$. Since $\operatorname{dim}(\partial(g \tilde{S}))=\operatorname{dim}(\tilde{S})<\operatorname{dim}(C)$, we have that $\Lambda(\Gamma) \backslash \partial(g \tilde{S})$ is a non-empty open subset of $\Lambda(\Gamma)$. Since $\Lambda_{\mathrm{r}}(\Gamma)$ is dense in $\Lambda(\Gamma)$, it follows that

$$
\Lambda_{\mathrm{r}}(\Gamma) \cap \operatorname{Viz}(g \tilde{E}) \neq \emptyset .
$$

Therefore there exists $h_{0} \in G_{\tilde{E}}$ such that $\operatorname{Viz}\left(g h_{0} e_{0}\right)=\left(g h_{0} e_{0}\right)^{+} \in \Lambda_{\mathrm{r}}(\Gamma)$. Hence there exist $r_{i} \rightarrow \infty$ such that $\overline{\mathbf{p}}\left(\mathcal{G}^{r_{i}}\left(g h_{0} e_{0}\right)\right)$ converges to a point in $\mathrm{T}^{1}(X)$. Then there exists a sequence $\left\{\gamma_{i}^{\prime}\right\} \subset \Gamma$ such that $\mathcal{G}^{r_{i}}\left(\gamma_{i}^{\prime} g h_{0} e_{0}\right) \rightarrow u$ for some $u \in \mathrm{T}^{1}\left(\mathbb{H}^{n}\right)$.

Let $B=T P$ be an admissible box centered at $u$. Let $\epsilon>0$ be such that $u \in B_{3 \epsilon-}$. Fix $k \in \mathbb{N}$ such that $r_{k}>r_{\epsilon}$ (see (2.17) $)$ such that for $\gamma^{\prime}=\gamma_{k}^{\prime}$, we have $\mathcal{G}^{r}\left(\gamma^{\prime} g h_{0} e_{0}\right) \in B_{2 \epsilon-}$. 
Since $\gamma_{i} h_{i} \rightarrow g, \mathcal{G}^{r}\left(\gamma^{\prime} \gamma_{i} h_{i} h_{0} e_{0}\right) \in B_{\epsilon-}$ for all $i \geq i_{0}$ for some $i_{0}$. Since $h_{i} h_{0} e_{0} \in$ $\tilde{E}$, by (2.10) $t_{i} \in T \cap \mathcal{G}^{r}\left(\gamma^{\prime} \gamma_{i} \tilde{E}\right)$ for all $i \geq i_{0}$. Therefore,

$$
\left(\Gamma T \cap \mathcal{G}^{r} \tilde{E}\right) \supset\left\{\left(\gamma^{\prime} \gamma_{i}\right)^{-1} t_{i}: i \geq i_{0}\right\} .
$$

We claim that for any $i \in \mathbb{N}$,

$$
\Gamma_{\tilde{E}} \gamma_{i}^{-1}\left(\gamma^{\prime}\right)^{-1} t_{i} \neq \Gamma_{\tilde{E}} \gamma_{j}^{-1}\left(\gamma^{\prime}\right)^{-1} t_{j} \text {, for all but finitely many } j .
$$

To see this, since $\mathbf{p}$ is injective on $T$, if $t_{i} \neq t_{j}$, then $\Gamma t_{i} \neq \Gamma t_{j}$, and hence (2.30) holds. If $t_{i}=t_{j}$, then it follows from (2.28) as $\Gamma \cap G_{\left(\gamma^{\prime}\right)^{-1} t_{i}}$ is finite. Combining (2.29) and (2.30), we get that

$$
\#\left(\Gamma_{\tilde{E}} \backslash\left(\Gamma T \cap \mathcal{G}^{r} \tilde{E}\right)\right)=\infty .
$$

We observe that if $t \in T \cap \mathcal{G}^{r}(E)$, then $\Gamma_{\tilde{E}} \backslash\left(\Gamma t \cap \mathcal{G}^{r} \tilde{E}\right)=\bar{\Gamma}_{r, t}^{-1} t$. If $\left|\mu_{E}^{\mathrm{PS}}\right|<\infty$, then by (2.19) of Corollary 2.19

$$
\#\left(\Gamma_{\tilde{E}} \backslash\left(\Gamma T \cap \mathcal{G}^{r} \tilde{E}\right)\right) \leq \sum_{t \in T \cap \mathcal{G}^{r}(E)} \#\left(\bar{\Gamma}_{r, t}\right)<\infty,
$$

which is a contradiction.

Remark 2.22. (1) Theorem 2.21 holds for $\Gamma$ Zariski dense: since $\Gamma \subset G_{C}$ and $G_{C}$ is Zariski closed, we have $C=\partial \mathbb{H}^{n}$ for $\Gamma$ Zariski dense.

(2) Theorem 2.21 holds in the case $\Lambda\left(\Gamma_{\tilde{S}}\right)=\partial \tilde{S}$, since $\tilde{S} \subset C$ in this case, and hence we have that either $\tilde{S}=C$ or $\operatorname{dim}(C)>\operatorname{dim}(\tilde{S})$.

\section{Equidistribution of $\mathcal{G}_{*}^{r} \mu_{E}^{\mathrm{Leb}}$}

3.1. BMS-measure and BR-measure on $T^{1}(X)$. As before, let $\Gamma$ be a nonelementary torsion-free discrete subgroup of $G$ and set $X:=\Gamma \backslash \mathbb{H}^{n}$. Let $\left\{\mu_{x}\right\}$ and $\left\{\mu_{x}^{\prime}\right\}$ be $\Gamma$-invariant conformal densities on $\partial \mathbb{H}^{n}$ of dimension $\delta_{\mu}$ and $\delta_{\mu^{\prime}}$, respectively. Following Roblin [31, we define a measure $m^{\mu, \mu^{\prime}}$ on $\mathrm{T}^{1}(X)$ associated to $\left\{\mu_{x}\right\}$ and $\left\{\mu_{x}^{\prime}\right\}$ as follows. Fix $o \in \mathbb{H}^{n}$. Then the map

$$
u \mapsto\left(u^{+}, u^{-}, \beta_{u^{-}}(o, \pi(u))\right)
$$

is a homeomorphism between $\mathrm{T}^{1}\left(\mathbb{H}^{n}\right)$ with

$$
\left(\partial \mathbb{H}^{n} \times \partial \mathbb{H}^{n} \backslash\left\{(\xi, \xi): \xi \in \partial \mathbb{H}^{n}\right\}\right) \times \mathbb{R} .
$$

Hence we can define a measure $\tilde{m}^{\mu, \mu^{\prime}}$ on $\mathrm{T}^{1}\left(\mathbb{H}^{n}\right)$ by

$$
d \tilde{m}^{\mu, \mu^{\prime}}(u)=e^{\delta_{\mu} \beta_{u^{+}}(o, \pi(u))} e^{\delta_{\mu^{\prime}} \beta_{u^{-}}(o, \pi(u))} d \mu_{o}\left(u^{+}\right) d \mu_{o}^{\prime}\left(u^{-}\right) d s,
$$

where $s=\beta_{u^{-}}(o, \pi(u))$. Note that $\tilde{m}^{\mu, \mu^{\prime}}$ is $\Gamma$-invariant. Hence it induces a locally finite measure $m^{\mu, \mu^{\prime}}$ on $\mathrm{T}^{1}(X)$ such that if $\mathbf{p}$ is injective on $\Omega \subset \mathrm{T}^{1}\left(\mathbb{H}^{n}\right)$, then

$$
m^{\mu, \mu^{\prime}}(\mathbf{p}(\Omega))=\tilde{m}^{\mu, \mu^{\prime}}(\Omega) .
$$

This definition is independent of the choice of $o \in \mathbb{H}^{n}$.

Two important conformal densities on $\mathbb{H}^{n}$ that we will consider are the PattersonSullivan density and the $G$-invariant (Lebesgue) density. 
3.1.1. Critical exponent $\delta_{\Gamma}$. We denote by $\delta_{\Gamma}$ the critical exponent of $\Gamma$ which is defined as the abscissa of convergence of a Poincaré series $\sum_{\gamma \in \Gamma} e^{-s d(o, \gamma(o))}$ for some $o \in \mathbb{H}^{n}$; that is, the series converges for $s>\delta_{\Gamma}$ and diverges for $s<\delta_{\Gamma}$, and the convergence property is independent of the choice of $o \in \mathbb{H}^{n}$.

As $\Gamma$ is non-elementary, we have $\delta_{\Gamma}>0$. Generalizing the work of Patterson [26] for $n=2$, Sullivan [36] constructed a $\Gamma$-invariant conformal density $\left\{\nu_{x}: x \in \mathbb{H}^{n}\right\}$ of dimension $\delta_{\Gamma}$ supported on $\Lambda(\Gamma)$, which is unique up to homothety and is called the Patterson-Sullivan density. From now on, we will simply write $\delta$ instead of $\delta_{\Gamma}$.

We denote by $\left\{m_{x}: x \in \mathbb{H}^{n}\right\}$ a $G$-invariant conformal density on the boundary $\partial \mathbb{H}^{n}$ of dimension $(n-1)$, which is unique up to homothety, and each $m_{x}$ is invariant under the maximal compact subgroup $G_{x}$. It will be called the Lebesgue density.

The measure $m^{\nu, \nu}$ on $\mathrm{T}^{1}(X)$ is called the Bowen-Margulis-Sullivan measure $m^{\text {BMS }}$ associated with $\left\{\nu_{x}\right\}$ ([5], 20], 37]):

$$
d m^{\operatorname{BMS}}(u)=e^{\delta \beta_{u^{+}}(o, \pi(u))} \cdot e^{\delta \beta_{u^{-}}(o, \pi(u))} d \nu_{o}\left(u^{+}\right) d \nu_{o}\left(u^{-}\right) d s .
$$

The measure $m^{\nu, m}$ is called the Burger-Roblin measure $m^{\mathrm{BR}}$ associated with $\left\{\nu_{x}\right\}$ and $\left\{m_{x}\right\}([6],[31)$ :

$$
d m^{\mathrm{BR}}(u)=e^{(n-1) \beta_{u^{+}}(o, \pi(u))} \cdot e^{\delta \beta_{u^{-}}(o, \pi(u))} d m_{o}\left(u^{+}\right) d \nu_{o}\left(u^{-}\right) d s .
$$

We note that the support of $m^{\mathrm{BMS}}$ and $m^{\mathrm{BR}}$ are given respectively by $\{u \in$ $\left.\mathrm{T}^{1}(X): u^{+}, u^{-} \in \Lambda(\Gamma)\right\}$ and $\left\{u \in \mathrm{T}^{1}(X): u^{-} \in \Lambda(\Gamma)\right\}$.

3.2. Relation to classification of measures invariant under horocycles. Burger [6] showed that for a convex cocompact hyperbolic surface $\Gamma \backslash \mathbb{H}^{2}$ with $\delta>$ $1 / 2, m^{\mathrm{BR}}$ is a unique ergodic horocycle invariant locally finite measure which is not supported on closed horocycles. Roblin extended Burger's result in much greater generality. By identifying the space $\Omega_{\mathcal{H}}$ of all unstable horospheres with $\partial \mathbb{H}^{n} \times \mathbb{R}$ by $\mathcal{H}^{+}(u) \mapsto\left(u^{-}, \beta_{u^{-}}(o, \pi(u))\right)$, one defines the measure $d \hat{\mu}(\mathcal{H})=d \nu_{o}(\xi) e^{\delta s} d s$ for $\mathcal{H}=(\xi, s)$. Then Roblin's theorem [31, Thm. 6.6] says that if $\left|m^{\mathrm{BMS}}\right|<\infty$, then $\hat{\mu}$ is the unique Radon $\Gamma$-invariant measure on $\Lambda_{\mathrm{r}}(\Gamma) \times \mathbb{R} \subset \Omega_{\mathcal{H}}$. This important classification result is not used in this article, but it suggests that the asymptotic distribution of expanding horospheres should be described by $m^{\mathrm{BR}}$.

3.3. Patterson-Sullivan and Lebesgue measures on $\tilde{E}, \mathcal{H}_{u}^{+}$and $E$. Let $\tilde{S}$ and $\tilde{E}$ be as in $\$ 2.3$. The following measures are special cases of the measures defined in $\$ 2.6$.

Fix $o \in \mathbb{H}^{n}$. Define the Borel measure $\mu_{\tilde{E}}^{\text {Leb }}$ on $\tilde{E}$ such that

$$
d \mu_{\tilde{E}}^{\mathrm{Leb}}(v)=e^{(n-1) \beta_{v^{+}}(o, \pi(v))} d m_{o}\left(v^{+}\right) .
$$

Since $\left\{m_{x}\right\}$ is a $G$-invariant conformal density on $\partial \mathbb{H}^{n}$, the measure $\mu_{\tilde{E}}^{\text {Leb }}$ is $G$ invariant; that is, $g_{*} \mu_{\tilde{E}}^{\mathrm{Leb}}=\mu_{g(\tilde{E})}^{\mathrm{Leb}}$. In particular, it is a $G_{\tilde{E}}$ invariant measure on $\tilde{E}$.

Define the Borel measure $\mu_{\tilde{E}}^{\mathrm{PS}}$ on $\tilde{E}$ such that

$$
d \mu_{\tilde{E}}^{\mathrm{PS}}(v)=e^{\delta \beta_{v^{+}}(o, \pi(v))} d \nu_{o}\left(v^{+}\right) .
$$

We note that $\mu_{\tilde{E}}^{\mathrm{PS}}$ is a $\Gamma$-invariant measure.

As described in 92.6 , we denote by $\mu_{E}^{\mathrm{Leb}}$ and $\mu_{E}^{\mathrm{PS}}$ the measures on $E=\mathbf{p}(\tilde{E})$ induced by $\mu_{\tilde{E}}^{\mathrm{Leb}}$ and $\mu_{\tilde{E}}^{\mathrm{PS}}$, respectively. Each of them is a pushforward of the corresponding locally finite measure on $\Gamma_{\tilde{E}} \backslash \tilde{E}$. 
As in $\$ 2.6 .1$, we have families of measures $\mu^{\mathrm{PS}}=\left\{\mu_{\mathcal{H}^{+}}^{\mathrm{PS}}\right\}$ and $\mu^{\mathrm{Leb}}=\left\{\mu_{\mathcal{H}^{+}}^{\mathrm{Leb}}\right\}$ on the unstable horospherical foliation satisfying

$$
\mu_{\mathcal{G}^{r}\left(\mathcal{H}^{+}\right)}^{\mathrm{PS}}\left(\mathcal{G}^{r}(F)\right)=e^{\delta r} \mu_{\mathcal{H}^{+}}^{\mathrm{PS}}(F) \text { and } \quad \mu_{\mathcal{G}^{r}\left(\mathcal{H}^{+}\right)}^{\mathrm{Leb}}\left(\mathcal{G}^{r}(F)\right)=e^{(n-1) r} \mu_{\mathcal{H}^{+}}^{\mathrm{Leb}}(F)
$$

for any Borel subset $F$ of $\mathbf{p}\left(\mathcal{H}^{+}\right)$.

3.4. Transverse measures for $m^{\mathrm{BMS}}$. For each measurable $T$ contained in a weak stable leaf of the geodesic flow on $\mathrm{T}^{1}\left(\mathbb{H}^{n}\right)$, called a transversal, define a measure $\lambda_{T}$ on $T$ by

$$
d \lambda_{T}(t)=e^{-\delta s} d \nu_{o}\left(t^{-}\right) d s,
$$

where $s=\beta_{t^{-}}(o, \pi(t))$. If $B=T P$ is any box and $p \in P$, then $(t p)^{-}=t^{-}$and $\mathcal{H}_{t p}^{+}=\mathcal{H}_{t}^{+}$, and hence $\beta_{(t p)^{-}}(o, \pi(t p))=\beta_{t^{-}}(o, \pi(t))$. Hence

$$
d \lambda_{T p}(t p)=d \lambda_{T}(t)
$$

that is, $\lambda_{T}$ is holonomy invariant, where the holonomy is given by $t \mapsto t p$.

Now for any $\Psi \in C(B)$, by (3.2)-(3.6), we have

$$
\begin{aligned}
\int_{B} f d m^{\mathrm{BMS}} & =\int_{T} \int_{P} \Psi(t p) d \mu_{\mathcal{H}_{t}^{+}}^{\mathrm{PS}}(t p) d \lambda_{T}(t), \\
\int_{B} f d m^{\mathrm{BR}} & =\int_{T} \int_{P} \Psi(t p) d \mu_{\mathcal{H}_{t}^{+}}^{\mathrm{Leb}}(t p) d \lambda_{T}(t) .
\end{aligned}
$$

\subsubsection{Backward admissible box.}

Lemma 3.1. For any $u \in \mathrm{T}^{1}\left(\mathbb{H}^{n}\right)$ and $\epsilon>0$, there exists a box $B=T P$ about $u$ such that

(1) $\left|\lambda_{T}\right|>0$, or equivalently $\nu_{o}\left(\left\{t^{-}: t \in T\right\}\right)>0$, and

(2) $\lim \sup _{r \rightarrow \infty} d\left(\mathcal{G}^{r}(t p), \mathcal{G}^{r}\left(t^{\prime} p\right)\right)<\epsilon$, for all $t, t^{\prime} \in T$ and $p \in P$.

Such a box $B$ as above will be called a backward admissible box with asymptotically $\epsilon$-thin transversals.

Proof. As in the proof of Lemma 2.17 there exists a relatively compact open neighborhood $P^{-}$of $u$ in $\mathcal{H}_{u}^{-}$such that $\nu_{o}\left(\left\{t^{-}: t \in P^{-}\right\}\right)>0$, and $\mathbf{p}$ is injective on a neighborhood of the closure of $P^{-}$. Let $r_{0}=-\log \left(\epsilon / 4 \operatorname{diam}\left(P^{-}\right)\right)$. Then $\operatorname{diam}\left(\mathcal{G}^{r_{0}}\left(P^{-}\right)\right)=\epsilon / 4$ and $\mathbf{p}$ is injective on a neighborhood of the closure of $\mathcal{G}^{r_{0}}\left(P^{-}\right)$. Let $T_{1}$ be an open relatively compact neighborhood of $\mathcal{G}^{r_{0}}\left(P^{-}\right)$in $\mathrm{Viz}^{-1}\left(u^{+}\right)$and $P_{1}$ be an open relatively compact neighborhood of $\mathcal{G}^{r_{0}}(u)$ in $\mathcal{H}_{\mathcal{G}^{r_{0}}}^{+}(u)$ such that $T_{1} P_{1}$ is a box about $\mathcal{G}^{r_{0}}(u)$ contained in a ball of radius $\epsilon / 2$ about $u$. Let $T=\mathcal{G}^{-r_{0}}\left(T_{1}\right)$ and $P=\mathcal{G}^{-r_{0}}\left(P_{1}\right)$. Then $B=T P$ has the required properties. Property (1) holds because

$$
\left\{t^{-}: t \in T\right\}=\left\{t^{-}: t \in T_{1}\right\} \supset\left\{t^{-}: t \in \mathcal{G}^{r_{0}}\left(P^{-}\right)\right\}=\left\{t^{-}: t \in P^{-}\right\} .
$$

For property (2), let $t_{1}=\mathcal{G}^{r_{0}}(t)$ and $t_{1}^{\prime}=\mathcal{G}^{r_{0}}\left(t^{\prime}\right)$ in $T_{1}$ and $p_{1}=\mathcal{G}^{r_{0}}(p) \in P_{1}$. Since $\left(t_{1} p_{1}\right)^{+}=\left(t_{1}^{\prime} p_{1}\right)^{+}$, for any $r>r_{0}$,

$$
d\left(\mathcal{G}^{r}(t p), \mathcal{G}^{r}\left(t^{\prime} p\right)\right)=d\left(\mathcal{G}^{r-r_{0}}\left(t_{1} p_{1}\right), \mathcal{G}^{r-r_{0}}\left(t_{1}^{\prime} p_{1}\right)\right) \leq d\left(t_{1} p_{1}, t_{1}^{\prime} p_{1}\right) \leq \epsilon .
$$


3.5. Mixing of the geodesic flow. We assume that $\left|m^{\mathrm{BMS}}\right|<\infty$ for the rest of this section. This implies that $\Gamma$ is of divergent type; that is, $\sum_{\gamma \in \Gamma} e^{-\delta d(o, \gamma o)}=\infty$ and the $\Gamma$-invariant conformal density of dimension $\delta$ is unique up to homothety (see [31, Coro. 1.8]).

Hence, up to homothety, $\nu_{x}$ is the weak-limit as $s \rightarrow \delta^{+}$of the family of measures

$$
\nu_{x, o}(s):=\frac{1}{\sum_{\gamma \in \Gamma} e^{-s d(o, \gamma o)}} \sum_{\gamma \in \Gamma} e^{-s d(x, \gamma o)} \delta_{\gamma o},
$$

where $\delta_{\gamma o}$ denotes the unit mass at $\gamma o$ for some $o \in \mathbb{H}^{n}$.

The most crucial ergodic theoretic result involved in this work is the mixing of geodesic flow which was obtained by Rudolph [32] for a geometrically finite $\Gamma$, and by Roblin 31], as well as Babillot [1, in a much greater generality:

Theorem 3.2 (Rudolph, Roblin, Babillot). For any $\Psi_{1} \in L^{2}\left(\mathrm{~T}^{1}(X), m^{\mathrm{BMS}}\right)$ and $\Psi_{2} \in L^{2}\left(\mathrm{~T}^{1}(X), m^{\mathrm{BMS}}\right)$,

$$
\lim _{r \rightarrow \infty} \int_{\mathrm{T}^{1}(X)} \Psi_{1}\left(\mathcal{G}^{r}(x)\right) \Psi_{2}(x) d m^{\mathrm{BMS}}(x)=\frac{1}{\left|m^{\mathrm{BMS}}\right|} m^{\mathrm{BMS}}\left(\Psi_{1}\right) \cdot m^{\mathrm{BMS}}\left(\Psi_{2}\right) .
$$

From this theorem, we derive the following result, which generalizes the corresponding result for PS-measures on unstable horospheres due to Roblin [31, Corollary 3.2$]$.

Theorem 3.3. For any $\Psi \in C_{c}\left(\mathrm{~T}^{1}(X)\right)$ and $f \in L^{1}\left(E, \mu_{E}^{\mathrm{PS}}\right)$,

$$
\lim _{r \rightarrow \infty} \int_{x \in E} \Psi\left(\mathcal{G}^{r}(x)\right) f(x) d \mu_{E}^{\mathrm{PS}}(x)=\frac{\mu_{E}^{\mathrm{PS}}(f)}{\left|m^{\mathrm{BMS}}\right|} \cdot m^{\mathrm{BMS}}(\Psi) .
$$

We will deduce the above statement from its following version.

Proposition 3.4. Let $\Psi \in L^{1}\left(\mathrm{~T}^{1}(X), m^{\mathrm{BMS}}\right)$ and $f \in L^{1}\left(E, \mu_{E}^{\mathrm{PS}}\right)$ both be nonnegative, bounded and vanish outside compact sets. Then for any $\epsilon>0$,

$$
\begin{aligned}
& \limsup _{r \rightarrow \infty} \int_{x \in E} \Psi\left(\mathcal{G}^{r}(x)\right) f(x) d \mu_{E}^{\mathrm{PS}}(x) \leq \frac{\mu_{E}^{\mathrm{PS}}(f)}{\left|m^{\mathrm{BMS}}\right|} \cdot m^{\mathrm{BMS}}\left(\Psi_{\epsilon}^{+}\right), \\
& \liminf _{r \rightarrow \infty} \int_{x \in E} \Psi\left(\mathcal{G}^{r}(x)\right) f(x) d \mu_{E}^{\mathrm{PS}}(x) \geq \frac{\mu_{E}^{\mathrm{PS}}(f)}{\left|m^{\mathrm{BMS}}\right|} \cdot m^{\mathrm{BMS}}\left(\Psi_{\epsilon}^{-}\right),
\end{aligned}
$$

where, for any $u \in \mathrm{T}^{1}\left(\mathbb{H}^{n}\right)$,

$$
\begin{aligned}
& \Psi_{\epsilon}^{+}(\mathbf{p}(u)):=\sup \left\{\Psi(\mathbf{p}(v)): d(v, u)<\epsilon, v \in \operatorname{Viz}^{-1}\left(u^{+}\right)\right\} \\
& \Psi_{\epsilon}^{-}(\mathbf{p}(u)):=\inf \left\{\Psi(\mathbf{p}(v)): d(v, u)<\epsilon, v \in \operatorname{Viz}^{-1}\left(u^{+}\right)\right\}
\end{aligned}
$$

Proof. By Lemma 3.1, there exists a finite open cover $\mathcal{B}$ of $\operatorname{supp}(f) \subset E \subset \mathrm{T}^{1}(X)$ consisting of backward admissible boxes $B$ with asymptotically $\epsilon$-thin transversals; we identify $B \subset \mathrm{T}^{1}\left(\mathbb{H}^{n}\right)$ with $\mathbf{p}(B)$. By considering a partition of unity subordinate to this cover, $f=\sum_{B \in \mathcal{B}} \phi_{B}$, where $\phi_{B} \in L^{1}\left(E, \mu_{E}^{\mathrm{PS}}\right)$ is a non-negative function whose support is contained in $\mathbf{p}(B)$. Therefore it is enough to prove (3.10) and (3.11) for $\phi_{B}$ in place of $f$ for each $B \in \mathcal{B}$. 
Fix any $B \in \mathcal{B}$. For each $[\gamma] \in \Gamma / \Gamma_{\tilde{E}}$, let $\phi_{\gamma}(w)=\phi_{B}(w)$ for all $w \in \gamma \tilde{E}$. By (2.14),

$$
\begin{aligned}
\mu_{E}^{\mathrm{PS}}\left(\phi_{B}\right) & =\sum_{[\gamma] \in \Gamma / \Gamma_{\tilde{E}}} \mu_{\gamma \tilde{E}}^{\mathrm{PS}}\left(\phi_{\gamma}\right) \text { and } \\
\int_{x \in E} \Psi\left(\mathcal{G}^{r}(x)\right) \phi_{B}(x) d \mu_{E}^{\mathrm{PS}}(x) & =\sum_{[\gamma] \in \Gamma / \Gamma_{\tilde{E}}} \int_{w \in \gamma \tilde{E} \cap B} \Psi\left(\mathcal{G}^{r}(w)\right) \phi_{\gamma}(w) d \mu_{\gamma \tilde{E}}^{\mathrm{PS}}(w) .
\end{aligned}
$$

Therefore to prove (3.10) and (3.11) for $\phi_{B}$ in place of $f$, it is enough to prove the following: for any $\gamma \in \Gamma$ and $\phi:=\phi_{\gamma} \in L^{1}\left(\gamma \tilde{E}, \mu_{\gamma \tilde{E}}^{\mathrm{PS}}\right)$ vanishing outside $\gamma \tilde{E} \cap B$, we have

$$
\begin{aligned}
& \limsup _{r \rightarrow \infty} \int_{w \in \gamma \tilde{E} \cap B} \Psi\left(\mathcal{G}^{r}(w)\right) \phi(w) d \mu_{\gamma \tilde{E}}^{\mathrm{PS}}(w) \leq \frac{\mu_{\gamma \tilde{E}}^{\mathrm{PS}}(\phi)}{\left|m^{\mathrm{BMS}}\right|} m^{\mathrm{BMS}}\left(\Psi_{\epsilon}^{+}\right), \\
& \liminf _{r \rightarrow \infty} \int_{w \in \gamma \tilde{E} \cap B} \Psi\left(\mathcal{G}^{r}(w)\right) \phi(w) d \mu_{\gamma \tilde{E}}^{\mathrm{PS}}(w) \geq \frac{\mu_{\gamma \tilde{E}}^{\mathrm{PS}}(\phi)}{\left|m^{\mathrm{BMS}}\right|} m^{\mathrm{BMS}}\left(\Psi_{\epsilon}^{-}\right) .
\end{aligned}
$$

Now we express $B=T P$. If $\gamma \tilde{E} \cap B=\emptyset$, then both sides of (3.13) are zero, and hence the claim is true. Otherwise, there exists $\left(t_{1}, p_{1}\right) \in T \times P$ such that $v:=t_{1} p_{1} \in \gamma \tilde{E}$. We recall that as in $\$ 2.3 .1, \xi_{v}: \mathcal{H}_{v}^{+} \backslash\left(\gamma \cdot \mathrm{Viz}^{-1}(\partial \tilde{S})\right) \rightarrow \gamma \tilde{E} \backslash\{-v\}$ and $q_{v}: \gamma \tilde{E} \backslash\{-v\} \rightarrow \mathcal{H}_{v}^{+} \backslash\left(\gamma \cdot \mathrm{Viz}^{-1}(\partial \tilde{S})\right)$ are differentiable inverses of each other.

Letting

$$
P_{1}=\left\{p \in P: \xi_{v}\left(t_{1} p\right) \in T p\right\}
$$

we claim that

$$
\gamma \tilde{E} \cap B=\left\{\xi_{v}\left(t_{1} p\right): p \in P_{1}\right\} .
$$

To see this, if $t p \in \gamma \tilde{E}$ for some $(t, p) \in T \times P$, then

$$
q_{v}(t p)=\mathcal{H}_{v}^{+} \cap \mathrm{Viz}^{-1}\left((t p)^{+}\right)=\mathcal{H}_{t_{1}}^{+} \cap \mathrm{Viz}^{-1}\left(p^{+}\right)=t_{1} p .
$$

Hence $\xi_{v}\left(t_{1} p\right)=t p$, and so $p \in P_{1}$. The opposite inclusion is obvious.

We define a map $\rho: T P \rightarrow \gamma \tilde{E}$ as follows:

$$
\rho(t p)=\xi_{v}\left(t_{1} p\right), \text { for all }(t, p) \in T \times P .
$$

For any $t \in T$, for the restricted map $\rho: t P \rightarrow \gamma \tilde{E}$, by (2.12) and (2.15), and since $(t p)^{+}=p^{+}=\rho(t p)^{+}$, we have

$$
d \mu_{\gamma \tilde{E}}^{\mathrm{PS}}(\rho(t p)) / d \mu_{\mathcal{H}_{t}^{+}}^{\mathrm{PS}}(t p)=e^{\beta_{p^{+}}(\pi(t p), \pi(\rho(t p)))} .
$$

In view of this, we define $\Phi \in L^{2}\left(\mathrm{~T}^{1}(X), \mu^{\mathrm{BMS}}\right)$ as follows: $\Phi(x)=0$ if $x \in X \backslash B$ and

$$
\Phi(t p)=\phi(\rho(t p)) e^{\beta_{p^{+}}(\pi(t p), \pi(\rho(t p)))} \text { if } x=t p \in B
$$

We note that

$$
\Phi(t p) \neq 0 \Rightarrow \rho(t p) \in B \Rightarrow p \in P_{1} .
$$

Also, for $t \in T$ and $p \in P_{1}$, we have $\{\rho(t p), t p\} \subset T p$. Since $\mathcal{G}^{r}(B)$ has $\epsilon$-thin transversals as $r \rightarrow \infty$ (see Lemma 3.1(2)),

$$
\limsup _{r \rightarrow \infty} d\left(\mathcal{G}^{r}(\rho(t p)), \mathcal{G}^{r}(t p)\right) \leq \epsilon \text { for all } p \in P_{1} .
$$


By Theorem 3.2 .

$$
\begin{aligned}
& \frac{1}{\left|m^{\mathrm{BMS}}\right|} m^{\mathrm{BMS}}\left(\Psi_{\epsilon}^{+}\right) \cdot m^{\mathrm{BMS}}(\Phi) \\
& =\lim _{r \rightarrow \infty} \int_{B} \Psi_{\epsilon}^{+}\left(\mathcal{G}^{r}(x)\right) \Phi(x) d m^{\mathrm{BMS}}(x) \\
& =\lim _{r \rightarrow \infty} \int_{t \in T}\left(\int_{p \in P_{1}} \Psi_{\epsilon}^{+}\left(\mathcal{G}^{r}(t p)\right) \Phi(t p) d \mu_{\mathcal{H}_{t}^{+}}^{\mathrm{PS}}(t p)\right) d \lambda_{T}(t) \\
& =\lim _{r \rightarrow \infty} \int_{t \in T}\left(\int_{p \in P_{1}} \Psi_{\epsilon}^{+}\left(\mathcal{G}^{r}(t p)\right) \phi(\rho(t p)) d \mu_{\gamma \tilde{E}}^{\mathrm{PS}}(\rho(t p))\right) d \lambda_{T}(t) \\
& \geq\left|\lambda_{T}\right| \cdot \limsup _{r \rightarrow \infty} \int_{w \in \gamma \tilde{E} \cap B} \Psi\left(\mathcal{G}^{r}(w)\right) \phi(w) d \mu_{\gamma \tilde{E}}^{\mathrm{PS}}(w),
\end{aligned}
$$

where (3.22) follows from (3.7) and (3.18), (3.23) follows from (3.15), (3.16) and (3.17), and to justify (3.24) we put $w=\rho(t p)$ and use (3.12) and (3.19).

By putting $\Psi(x)=1=\Psi_{\epsilon}^{+}(x)$ in (3.21)-(3.24) with equality in (3.24), we get

$$
m^{\mathrm{BMS}}(\Phi)=\left|\lambda_{T}\right| \cdot \mu_{\gamma \tilde{E}}^{\mathrm{PS}}(\phi)<\infty .
$$

Now (3.13) is deduced by comparing (3.20), (3.24) and (3.25), and noting that $\left|\lambda_{T}\right| \neq 0$ by the backward admissibility of $B$. Similarly we can deduce (3.14).

Proof of Theorem 3.3. Since both the sides of (3.9) are linear in $\Psi$, it is enough to prove it for $\Psi \geq 0$. Since $\Psi$ is uniformly continuous and $\left|m^{\mathrm{BMS}}\right|<\infty$,

$$
\lim _{\epsilon \rightarrow 0} m^{\mathrm{BMS}}\left(\Psi_{\epsilon}^{+}-\Psi_{\epsilon}^{-}\right)=0 .
$$

Therefore by Proposition 3.4, we have that (3.9) holds for all non-negative bounded measurable $f$ with compact support on $E$. Since the set of such $f$ 's is dense in $L^{1}\left(E, \mu_{E}^{\mathrm{PS}}\right)$ and both sides of (3.9) are linear and continuous in $f \in L^{1}\left(E, \mu_{E}^{\mathrm{PS}}\right)$, (3.9) holds for all $f \in L^{1}\left(E, \mu_{E}^{\mathrm{PS}}\right)$.

The following result is one of the basic tools developed in this article.

Theorem 3.5 (Transversal equidistribution). Let $f \in L^{1}\left(E, \mu_{E}^{\mathrm{PS}}\right)$ be such that $\mu_{E}^{\mathrm{PS}}\left(f_{\epsilon}^{+}-f_{\epsilon}^{-}\right) \rightarrow 0$ as $\epsilon \rightarrow 0$. Let $\psi \in C_{c}(T)$ for a transversal $T$ of a box $B$ (2.4). Then

$$
\lim _{r \rightarrow \infty} e^{-\delta r} \sum_{t \in T \cap \mathcal{G}^{r}(E)} \#\left(\bar{\Gamma}_{r, t}\right) \cdot \psi(t) \cdot f\left(\mathcal{G}^{-r}(t)\right)=\frac{\mu_{E}^{\mathrm{PS}}(f)}{\left|m^{\mathrm{BMS}}\right|} \cdot \lambda_{T}(\psi)
$$

where

$$
f_{\epsilon}^{+}(x)=\sup _{\{y \in E: d(y, x)<\epsilon\}} f(y) \text { and } f_{\epsilon}^{-}(x)=\inf _{\{y \in E: d(y, x)<\epsilon\}} f(y),
$$

the transverse measure $\lambda_{T}$ is defined by (3.6) and $\bar{\Gamma}_{r, t}$ is defined by (2.27).

Proof. Since both sides of (3.26) are linear in $f$ and in $\psi$, without loss of generality we may assume that $f \geq 0$ and $\psi \geq 0$. By Lemma 2.17 $\operatorname{supp}(\psi)$ can be covered by finitely many admissible boxes. By a partition of unity argument, in view of Remark 2.5. we may assume without loss of generality that $T$ is a transversal of an admissible box $B$. 
Let $\epsilon_{0}>0$ be such that $\mathbf{p}$ is injective on $B_{\epsilon_{0+}}$ and that $\psi$ vanishes outside $T_{\epsilon_{0}-}$. We extend $\psi$ to a continuous function on $T_{\epsilon_{0}}$ by putting $\psi=0$ on $T_{\epsilon_{0}}+T$. Since $B$ is admissible, due to Lemma 2.15, if we define

$$
\Psi(t p)=\psi(t) / \mu_{\mathcal{H}_{t}^{+}}^{\mathrm{PS}}(t P), \text { for all }(t, p) \in T_{\epsilon_{0}+} \times P,
$$

then $\Psi$ is a bounded continuous function on $B_{\epsilon_{0}}+$ vanishing outside $B_{\epsilon_{0}-}$. If $\Psi_{\epsilon}^{ \pm} \in$ $C\left(B_{\epsilon+}\right)$ are defined as in (3.12) for $0<\epsilon \leq \epsilon_{0}$, then

$$
\lim _{\epsilon \rightarrow 0}\left\|\Psi_{\epsilon}^{+}-\Psi_{\epsilon}^{-}\right\|_{\infty}=0
$$

By Proposition 3.4,

$$
\begin{aligned}
\limsup _{r \rightarrow \infty} \int_{E} \Psi_{\epsilon}^{+}\left(\mathcal{G}^{r}(v)\right) f_{\epsilon}^{+}(v) d \mu_{E}^{\mathrm{PS}}(v) & \leq \frac{\mu_{E}^{\mathrm{PS}}\left(f_{\epsilon}^{+}\right) m^{\mathrm{BMS}}\left(\Psi_{\epsilon}^{+}\right)}{\left|m^{\mathrm{BMS}}\right|}, \\
\liminf _{r \rightarrow \infty} \int_{E} \Psi_{\epsilon}^{-}\left(\mathcal{G}^{r}(v)\right) f_{\epsilon}^{-}(v) d \mu_{E}^{\mathrm{PS}}(v) & \geq \frac{\mu_{E}^{\mathrm{PS}}\left(f_{\epsilon}^{-}\right) m^{\mathrm{BMS}}\left(\Psi_{\epsilon}^{-}\right)}{\left|m^{\mathrm{BMS}}\right|} .
\end{aligned}
$$

Since $m^{\mathrm{BMS}}\left(B_{\epsilon_{0}+}\right)<\infty$, by (3.27), we have that $m^{\mathrm{BMS}}\left(\Psi_{\epsilon}^{+}-\Psi_{\epsilon}^{-}\right) \rightarrow 0$ as $\epsilon \rightarrow 0$. By our assumption, $\mu_{E}^{\mathrm{PS}}\left(\left|f_{\epsilon}^{ \pm}-f\right|\right) \rightarrow 0$ as $\epsilon \rightarrow 0$. Therefore by Proposition 2.18 and (3.28),

$$
\lim _{r \rightarrow \infty} e^{-\delta r} \sum_{t \in T \cap \mathcal{G}^{r}(E)} \#\left(\bar{\Gamma}_{r, t}\right) \cdot \psi(t) \cdot f\left(\mathcal{G}^{-r}(t)\right)=\frac{\mu_{E}^{\mathrm{PS}}(f) m^{\mathrm{BMS}}(\Psi)}{\left|m^{\mathrm{BMS}}\right|} .
$$

Also,

$$
m^{\mathrm{BMS}}(\Psi)=\int_{T} d \mu_{T}(t)\left(\int_{t P} \Psi(t p) d \mu_{\mathcal{H}_{t}^{+}}^{\mathrm{PS}}\right)=\lambda_{T}(\psi)
$$

Now we state and prove the main equidistribution result of this article which is more general than Theorem 1.8 .

Theorem 3.6. Let $f \in L^{1}\left(E, \mu_{E}^{\mathrm{PS}}\right)$ such that $\mu_{E}^{\mathrm{PS}}\left(f_{\epsilon}^{+}-f_{\epsilon}^{-}\right) \rightarrow 0$ as $\epsilon \rightarrow 0$. Let $\Psi \in C_{c}\left(\mathrm{~T}^{1}(X)\right)$. Then

$$
\lim _{r \rightarrow \infty} e^{(n-1-\delta) r} \int_{u \in E} \Psi\left(\mathcal{G}^{r}(u)\right) f(u) d \mu_{E}^{\mathrm{Leb}}(u)=\frac{\mu_{E}^{\mathrm{PS}}(f)}{\left|m^{\mathrm{BMS}}\right|} m^{\mathrm{BR}}(\Psi) .
$$

In particular, the result applies to $f=\chi_{F}$ for a Borel measurable $F \subset E$ such that $\mu_{E}^{\mathrm{PS}}\left(F_{\epsilon_{1}}\right)<\infty$ for some $\epsilon_{1}>0$ and $\mu_{E}^{\mathrm{PS}}(\partial F)=0$.

Proof. By Lemma 2.17, the boxes admissible with respect to $\left\{\mu_{\mathcal{H}^{+}}^{\mathrm{PS}}\right\}$ form a basis of open sets in $\mathrm{T}^{1}(X)$. By a partition of unity argument, without loss of generality we may assume that $\operatorname{supp}(\Psi) \subset B$ for an admissible box $B=T P$. Let $\epsilon_{0}>0$ be such that $\Psi=0$ outside $B_{\epsilon_{0}-}$. For $0<\epsilon \leq \epsilon_{0}$, let $\Psi_{\epsilon}^{ \pm}$be defined as in (2.18). Then

$$
\lim _{\epsilon \rightarrow 0}\left\|\Psi_{\epsilon}^{+}-\Psi_{\epsilon}^{-}\right\|_{\infty}=0
$$

For $t \in T_{\epsilon_{0}}$ and $\epsilon>0$, define $\psi_{\epsilon}^{ \pm}(t)=\int_{t P} \Psi_{\epsilon}^{ \pm} d \mu_{\mathcal{H}_{t}^{+}}^{\text {Leb }}$ By Lemma 2.15, $\psi_{\epsilon}^{ \pm} \in$ $C_{c}(T)$ for any $0<\epsilon<\epsilon_{0} / 2$. 
For the conformal density, $\left\{\mu_{x}\right\}=\left\{m_{x}\right\}$, we have $\delta_{\mu}=n-1$, and by multiplying all the terms in the conclusion of Corollary 2.14 by $e^{-\delta r}$, for $r>r_{\epsilon}$ (see (2.17)), we get

$$
\begin{aligned}
& \left(e^{-(n-1) \epsilon}\right) e^{-\delta r} \sum_{t \in T \cap \mathcal{G}^{r}(E)} \#\left(\bar{\Gamma}_{r, t}\right) \cdot \psi_{\epsilon}^{-}(t) \cdot f_{\epsilon}^{-}\left(\mathcal{G}^{-r}(t)\right) \\
\leq & e^{(n-1-\delta) r} \int_{E} \Psi\left(\mathcal{G}^{r}(u)\right) f(u) d \mu_{E}^{\mathrm{Leb}}(u) \\
\leq & \left(e^{(n-1) \epsilon}\right) e^{-\delta r} \sum_{t \in T \cap \mathcal{G}^{r}(E)} \#\left(\bar{\Gamma}_{r, t}\right) \cdot \psi_{\epsilon}^{+}(t) \cdot f_{\epsilon}^{+}\left(\mathcal{G}^{-r}(t)\right) .
\end{aligned}
$$

Define $\psi(t):=\int_{t P} \Psi(t p) d \mu_{\mathcal{H}_{t}^{+}}^{\mathrm{Leb}}$ for all $t \in T$. Then $\lambda_{T}(\psi)=m^{\mathrm{BR}}(\Psi)$ and $\lambda_{T}\left(\psi_{\epsilon}^{ \pm}\right)=m^{\mathrm{BR}}\left(\Psi_{\epsilon}^{ \pm}\right)$. Since $m^{\mathrm{BMS}}\left(B_{\epsilon_{0}+}\right)<\infty$, by (3.29) ,

$$
\lambda_{T}\left(\psi^{+}\right)-\lambda_{T}\left(\psi^{-}\right)=m^{\mathrm{BR}}\left(\Psi_{\epsilon}^{+}-\Psi_{\epsilon}^{-}\right) \rightarrow 0, \text { as } \epsilon \rightarrow 0 .
$$

Also, since $\mu_{E}^{\mathrm{PS}}\left(f_{\epsilon}^{+}-f_{\epsilon}^{-}\right) \rightarrow 0$, by Theorem 3.5

$$
\lim _{r \rightarrow \infty} e^{(n-1-\delta) r} \int_{E} \Psi\left(\mathcal{G}^{r}(u)\right) f(u) d \mu_{E}^{\mathrm{Leb}}(u)=\frac{\mu_{E}^{\mathrm{PS}}(f) \lambda_{T}(\psi)}{\left|m^{\mathrm{BMS}}\right|} .
$$

Since $\lambda_{T}(\psi)=m^{\mathrm{BR}}(\Psi)$, we prove the claim.

In the particular case of $f=\chi_{F}$, we have

$$
\inf _{\epsilon>0} f_{\epsilon}^{+}=\chi_{\bar{F}} \quad \text { and } \quad \sup _{\epsilon>0} f_{\epsilon}^{-}=\chi_{\operatorname{int}(F)}, \text { and }
$$

if $\mu_{E}^{\mathrm{PS}}\left(f_{\epsilon_{1}}^{+}\right)=\mu_{E}^{\mathrm{PS}}\left(F_{\epsilon_{1}}\right)<\infty$, then $\lim _{\epsilon \rightarrow 0} \mu_{E}^{\mathrm{PS}}\left(f_{\epsilon}^{+}-f_{\epsilon}^{-}\right)=\mu_{E}^{\mathrm{PS}}(\partial F)$.

The idea of the above proof was influenced by the work of Schapira 34 .

Our proof also yields the following variation of Theorem 3.6

Theorem 3.7. Let $\tilde{F} \subset \tilde{E}$ be a Borel subset such that $\mu_{\tilde{E}}^{\mathrm{PS}}\left(\tilde{F}_{\epsilon}\right)<\infty$ for some $\epsilon>0$ and $\mu_{\tilde{E}}^{\mathrm{PS}}(\partial \tilde{F})=0$. Then for any $\psi \in C_{c}\left(\mathrm{~T}^{1}\left(\Gamma \backslash \mathbb{H}^{n}\right)\right)$,

$$
\lim _{t \rightarrow+\infty} e^{(n-1-\delta) t} \cdot \int_{\tilde{F}} \psi\left(\mathcal{G}^{t}(v)\right) d \mu_{\tilde{E}}^{\mathrm{Leb}}(v)=\frac{\mu_{\tilde{E}}^{\mathrm{PS}}(\tilde{F})}{\left|m^{\mathrm{BMS}}\right|} \cdot m^{\mathrm{BR}}(\psi) .
$$

\subsection{Integrability of the base eigenfunction $\phi_{0}$.}

Proof of Theorem 1.17, We want to prove equivalence of the following:

(11) $\phi_{0} \in L^{1}\left(\Gamma \backslash \mathbb{H}^{n}, d \operatorname{Vol}_{\text {Riem }}\right)$;

(2) $\left|m^{\mathrm{BR}}\right|<\infty$;

(3i) $\Gamma$ is a lattice in $G$.

The pushforward of $m^{\mathrm{BR}}$ from $\Gamma \backslash \mathrm{T}^{1}\left(\mathbb{H}^{1}\right)$ to $\Gamma \backslash \mathbb{H}^{n}$ is the measure corresponding to $\phi_{0} d \mathrm{Vol}_{\text {Riem }}$ (see [17, Lemma 6.7]). Therefore (11) and (2) are equivalent.

To prove that (2) implies (3), suppose that $\left|m^{\mathrm{BR}}\right|<\infty$. Since the left $G$-action on $\mathrm{T}^{1}\left(\mathbb{H}^{n}\right)$ is transitive, we may identify $\mathrm{T}^{1}\left(\Gamma \backslash \mathbb{H}^{n}\right)$ with $\Gamma \backslash G / M$ for a compact subgroup $M$. We lift the measure $m^{\mathrm{BR}}$ to a measure $m$ on $\Gamma \backslash G$ as follows: for any $f \in C_{c}(\Gamma \backslash G)$, we define $m(f)=m^{\mathrm{BR}}(\bar{f})$, where $\bar{f}(\Gamma g M)=\int_{x \in M} f(\Gamma g x) d x$, where $d x$ is the probability Haar measure on $M$. Denote by $U$ the horospherical subgroup of $G$ whose orbits in $G$ project to the unstable horospheres in $\mathrm{T}^{1}\left(\mathbb{H}^{n}\right)$. 
Then $M$ normalizes $U$ and any unimodular proper closed subgroup of $G$ containing $U$ is contained in the subgroup $M U$. As $m$ is invariant under $G_{\mathcal{H}^{+}}$for any unstable horosphere $\mathcal{H}^{+}$, it follows that $m$ is a $U$-invariant finite measure on $\Gamma \backslash G$. By Ratner's theorem [30, any ergodic component, say, $\lambda$, of $m$ is a homogeneous measure in the sense that $\lambda$ is an $H$-invariant finite measure supported on a closed orbit $x_{0} H$ for some $x_{0} \in \Gamma \backslash G$ and a unimodular closed subgroup $H$ of $G$ containing $U$. If $H \neq G$, then $H \subset M U$ and $\Gamma \cap H$ is cocompact in $H$. It follows by a theorem of Bieberbach ([4, Theorem 2.25]) that $\Gamma \cap U$ is cocompact in $U$. Hence $H=U$. Thus we can write $m=m_{1}+m_{2}$, where $m_{1}$ is $G$-invariant and $m_{2}$ is supported on a union of compact $U$-orbits.

If $m_{1}=0$, then $m=m_{2}$, and hence the projection of the support of $m^{\mathrm{BR}}$ in $\mathrm{T}^{1}\left(\mathbb{H}^{n}\right)$ is a union of compact unstable horospheres. It follows that the PattersonSullivan density is concentrated on the set of parabolic fixed points of $\Gamma$, which is a contradiction.

If $m_{1} \neq 0$, then $m_{1}$ is a finite $G$-invariant measure on $\Gamma \backslash G$; that is, $\Gamma$ is a lattice in $G$. Hence (2) implies (3).

If $\Gamma$ is a lattice, then $\left\{\nu_{x}\right\}=\left\{m_{x}\right\}$ up to a constant multiple. Hence $m^{\text {BR }}$ is the projection of a finite $G$-invariant measure of $\Gamma \backslash G$ to $T^{1}\left(\Gamma \backslash \mathbb{H}^{n}\right)$. Hence (3) implies (2).

\section{Geometric finiteness of Closed totally GeOdesic immersions}

4.1. Parabolic fixed points and minimal subspaces. Let $\Gamma$ be a torsion-free discrete subgroup of $G$.

Definition 4.1. An element $g \in G$ is called parabolic if $\operatorname{Fix}(g):=\left\{\xi \in \partial \mathbb{H}^{n}: g \xi=\right.$ $\xi\}$ is a singleton set. An element $\xi \in \partial \mathbb{H}^{n}$ is called a parabolic fixed point of $\Gamma$ if there exists a parabolic element $\gamma \in \Gamma$ such that $\operatorname{Fix}(\gamma)=\{\xi\}$. Note that if $\xi$ is a parabolic fixed point for $\Gamma$, then $\xi \in \Lambda(\Gamma)$. Let $\Lambda_{\mathrm{p}}(\Gamma)$ denote the set of parabolic fixed points of $\Gamma$.

Let $\xi \in \Lambda_{\mathrm{p}}(\Gamma)$. In order to analyze the action of $\Gamma_{\xi}$ on $\partial \mathbb{H}^{n} \backslash\{\xi\}$, it is convenient to use the upper half space model $\mathbb{R}_{+}^{n}=\left\{(x, y): x \in \mathbb{R}^{n-1}, y>0\right\}$ for $\mathbb{H}^{n}$, where $\xi$ corresponds to $\infty$ and $\partial \mathbb{H}^{n} \backslash\{\xi\}$ corresponds to $\partial \mathbb{R}_{+}^{n}=\left\{(x, 0): x \in \mathbb{R}^{n-1}\right\}$. The subgroup $\Gamma_{\infty}$ acts properly discontinuously via affine isometries on $\partial \mathbb{H}^{n} \backslash\{\infty\} \cong$ $\mathbb{R}^{n-1}$. At this stage we will treat $\mathbb{R}^{n-1}$ only as an affine space, and we will choose its origin 0 later. Moreover, the action of $\Gamma_{\infty}$ preserves every horosphere $\mathbb{R}^{n-1} \times\{y\}$, where $y>0$, based at $\infty$.

By a theorem of Bieberbach (4, 2.2.5]), $\Gamma_{\infty}$ contains a normal abelian subgroup of finite index, say $\Gamma_{\infty}^{\prime}$. By [4, 2.1.5], any (non-empty) $\Gamma_{\infty}^{\prime}$-invariant affine subspace of $\mathbb{R}^{n-1}$ contains a (non-empty) minimal $\Gamma_{\infty}^{\prime}$-invariant affine subspace; we call such an affine subspace a $\Gamma_{\infty}^{\prime}$-minimal subspace. By [4, 2.2.6], $\Gamma_{\infty}^{\prime}$ acts cocompactly via translations on any $\Gamma_{\infty}^{\prime}$-minimal subspace. Moreover, any two $\Gamma_{\infty}^{\prime}$-minimal subspaces are parallel, and if $v_{1}$ and $v_{2}$ belong to any two $\Gamma_{\infty}^{\prime}$-minimal subspaces, then $\gamma v_{1}-\gamma v_{2}=v_{1}-v_{2}$ for all $\gamma \in \Gamma_{\infty}^{\prime}$. Let $\operatorname{rank}\left(\Gamma_{\infty}\right)$ denote the rank of the (torsion-free) $\mathbb{Z}$-module $\Gamma_{\infty}^{\prime}$; it is independent of the choice of $\Gamma_{\infty}^{\prime}$, and it equals the dimension of a $\Gamma_{\infty}^{\prime}$-minimal subspace.

Definition 4.2. A parabolic fixed point $\xi \in \Lambda_{\mathrm{p}}(\Gamma)$ is said to be bounded if $\Gamma_{\xi} \backslash(\Lambda(\Gamma) \backslash\{\xi\})$ is compact. Denote by $\Lambda_{\mathrm{bp}}(\Gamma)$ the set of all bounded parabolic 
fixed points for $\Gamma$. Therefore $\infty \in \Lambda_{\mathrm{bp}}(\Gamma)$ if and only if $\infty \in \Lambda_{\mathrm{p}}(\Gamma)$ and

$$
\Lambda(\Gamma) \backslash\{\infty\} \subset\left\{x \in \mathbb{R}^{n-1}: d_{\text {Euc }}(x, L) \leq r_{0}\right\},
$$

for some $r_{0}>0$, where $L$ is a $\Gamma_{\infty}^{\prime}$-minimal subspace.

4.2. On geometric finiteness of $\Gamma_{\tilde{S}}$. For the rest of this section, let $\tilde{S}$ be a proper connected totally geodesic subspace of $\mathbb{H}^{n}$ such that the natural projection map $\Gamma_{\tilde{S}} \backslash \tilde{S} \rightarrow X=\Gamma \backslash \mathbb{H}^{n}$ is proper or, equivalently, the map $\Gamma_{\tilde{S}} \backslash G_{\tilde{S}} \rightarrow \Gamma \backslash G$ is proper or, equivalently, $\Gamma G_{\tilde{S}}$ is closed in $G$. Since $\tilde{S}$ is totally geodesic, the geometric boundary $\partial \tilde{S}$ is the intersection of $\partial \mathbb{H}^{n}$ with the closure of $\tilde{S}$ in $\overline{\mathbb{H}^{n}}$.

Proposition 4.3. Let $\infty \in \Lambda_{\mathrm{p}}(\Gamma) \cap \partial \tilde{S}$. Let $L$ be a $\Gamma_{\infty}^{\prime}$-minimal subspace of $\partial \mathbb{H}^{n} \backslash\{\infty\} \cong \mathbb{R}^{n-1}$ and choose the origin $0 \in L$. Then the intersection of $L$ with the (parallel) translate of the affine subspace $\partial \tilde{S} \backslash\{\infty\}$ through 0 is a $\left(\Gamma_{\infty}^{\prime} \cap G_{\tilde{S}}\right)$ minimal subspace.

Proof. Let $\Gamma^{\prime}=\Gamma_{\infty}^{\prime}, \Delta=\Gamma^{\prime} \cap G_{\tilde{S}}$, and the affine subspace $F=\partial \tilde{S} \backslash\{\infty\}$. Since $\Delta F=F$, let $v$ belong to a $\Delta$-minimal subspace of $F$. Since $v$ and 0 belong to two $\Delta$ minimal subspaces, $\gamma v-\gamma 0=v-0=v$. Since $\gamma v \in F$, we have $\gamma 0=\gamma v-v \in F-v$. Since $0 \in F-v$, we have $\gamma 0 \in \gamma(F-v) \cap(F-v)$. Now $\gamma(F-v)$ and $\gamma F=F$ are parallel. Therefore $F-v$ and $\gamma(F-v)$ are parallel, and since they intersect, $\gamma(F-v)=F-v$. Thus $\Delta(F-v)=F-v$. Therefore, $\Delta$-action preserves $L_{0}:=L \cap(F-v)$. We want to prove that $\Gamma^{\prime} \cap G_{\tilde{S}}$ acts cocompactly on $L_{0}$.

Since $\infty \in \Lambda_{\mathrm{p}}(\Gamma)$, by [4, Lemma 3.2.1] $\Gamma_{\infty}$ consists of parabolic elements of $G_{\infty}$. That is, $\Gamma_{\infty} \subset M N$, where $N$ is the maximal unipotent subgroup of $G$ which acts transitively on $\mathbb{R}^{n-1}=\partial \mathbb{H}^{n} \backslash\{\infty\}$ via translations and $M$ is a compact subgroup of $G$ normalizing $N$ that acts on $\mathbb{R}^{n-1}$ by Euclidean isometries fixing 0 . Let $U=\{g \in N: g L=L\}$. Then $U$ acts transitively on $L$ by translations. Since $0 \in L$ and $\Gamma^{\prime}$ acts cocompactly on $L$ via translations, the connected component of the Zariski closure of $\Gamma^{\prime}$ in $G$ is a connected abelian subgroup of the form $M_{L} U$, where $M_{L} \subset M$ and $M_{L}$ acts trivially on $L$.

Since $\Gamma^{\prime} \backslash L$ is a compact Euclidean torus, the closure of the image of $L_{0}$ in $\Gamma^{\prime} \backslash L$ equals the image of an affine subspace, say $L_{1}$, of $L$. Thus $\overline{\Gamma^{\prime} L_{0}}=\Gamma^{\prime} L_{1}$. For $i=0,1$, let $U_{i}=\left\{u \in U: u L_{i}=L_{i}\right\}$. Then $U_{i}$ acts transitively on $L_{i}$, and $\overline{\Gamma^{\prime} M_{L} U_{0}}=\Gamma^{\prime} M_{L} U_{1}$. Therefore the identity component of $\overline{\Gamma^{\prime} U_{0}}$ is of the form $M_{1} U_{1}$, where $M_{1} \subset M_{L}$ and $\left(\Gamma^{\prime} \cap M_{1} U_{1}\right) \backslash M_{1} U_{1}$ is compact. In particular, $\Gamma^{\prime} \cap M_{1} U_{1}$ acts cocompactly on $L_{1}$.

By our assumption $\Gamma G_{\tilde{S}}$ is a closed subset of $G$. Therefore $\overline{\Gamma^{\prime} U_{0}} \subset \Gamma G_{\tilde{S}}$. Since $G_{\tilde{S}}$ is the identity component in $\Gamma G_{\tilde{S}}$, we have $M_{1} U_{1} \subset G_{\tilde{S}}$. It follows that $U_{1}$ preserves $L_{0}$. Since $U_{1}$ acts transitively on $L_{1}, L_{1} \subset L_{0}$; hence $L_{1}=L_{0}$. In particular, $\Gamma^{\prime} \cap M_{1} U_{1}$ acts cocompactly on $L_{0}$. Therefore $\Delta=\Gamma^{\prime} \cap G_{\tilde{S}}$ acts cocompactly on $L_{0}$.

Proposition 4.4. Let $\infty \in \Lambda_{\mathrm{bp}}(\Gamma) \cap \partial \tilde{S}$ and $\Gamma_{\tilde{S}}:=\Gamma \cap G_{\tilde{S}}$. Then

$$
\begin{cases}\infty \in \Lambda_{\mathrm{bp}}\left(\Gamma_{\tilde{S}}\right) & \text { if } \Gamma_{\infty} \cap \Gamma_{\tilde{S}} \text { is infinite, } \\ \infty \notin \Lambda\left(\Gamma_{\tilde{S}}\right) & \text { if } \Gamma_{\infty} \cap \Gamma_{\tilde{S}} \text { is finite, hence trivial. }\end{cases}
$$

Proof. Let the notation be as in Proposition 4.3. Since $\infty \in \Lambda_{\mathrm{bp}}(\Gamma)$, by (4.1), $\Lambda(\Gamma) \backslash\{\infty\}$ is contained in a bounded neighborhood of $L$, and hence in a bounded neighborhood of $L+v$. Therefore $(\Lambda(\Gamma) \backslash\{\infty\}) \cap \partial \tilde{S}$ is contained in a bounded 
neighborhood of $L+v$ intersected with $F=\partial \tilde{S} \backslash\{\infty\}$, and hence in a bounded neighborhood of $L_{0}=L \cap(F-v)$ as well. By Proposition 4.3, $L_{0}$ is a $\left(\Gamma_{\tilde{S}} \cap \Gamma^{\prime}\right)$ minimal subspace. Now if $\Gamma_{\infty} \cap \Gamma_{\tilde{S}}$ is infinite, or equivalently $\infty \in \Lambda_{\mathrm{p}}\left(\Gamma_{\tilde{S}}\right)$, then $\infty \in \Lambda_{\mathrm{bp}}\left(\Gamma_{\tilde{S}}\right)$.

Suppose that $\Gamma_{\infty} \cap \Gamma_{\tilde{S}}$ is finite. Then $L_{0}$ is a singleton set. Therefore $\Lambda\left(\Gamma_{\tilde{S}}\right) \backslash\{\infty\}$ is contained in a bounded subset of $\partial \tilde{S} \backslash\{\infty\}$. Then $\infty \in \partial \tilde{S}$ is isolated from $\Lambda\left(\Gamma_{\tilde{S}}\right)$. Since the limit set of a non-elementary hyperbolic group is perfect, it follows that $\Gamma_{\tilde{S}}$ is elementary, and hence $\Gamma_{\tilde{S}}$ is either parabolic or loxodromic. Now suppose that $\infty \in \Lambda\left(\Gamma_{\tilde{S}}\right)$. In the parabolic case $\Lambda\left(\Gamma_{\tilde{S}}\right)=\{\infty\}=\Lambda_{\mathrm{p}}\left(\Gamma_{\tilde{S}}\right)$, contradicting the assumption that $\Gamma_{\infty} \cap \Gamma_{\tilde{S}}=\{e\}$. In the loxodromic case, $\infty \in \Lambda_{\mathrm{r}}\left(\Gamma_{\tilde{S}}\right) \subset \Lambda_{\mathrm{r}}(\Gamma)$, contradicting the assumption that $\infty \in \Lambda_{\mathrm{p}}(\Gamma)$.

Lemma 4.5. We have

$$
\Lambda_{\mathrm{r}}(\Gamma) \cap \partial \tilde{S}=\Lambda_{\mathrm{r}}\left(\Gamma_{\tilde{S}}\right) .
$$

Proof. Let $\xi \in \Lambda_{\mathrm{r}}(\Gamma) \cap \partial \tilde{S}$. As $\tilde{S}$ is totally geodesic, there exists a geodesic ray, say, $\beta$, lying in $\tilde{S}$ pointing toward $\xi$. Since $\xi$ is a radial limit point, $\Gamma \beta$ accumulates on a compact subset of $\mathbb{H}^{n}$. By the assumption that the natural projection map $\Gamma_{\tilde{S}} \backslash \tilde{S} \rightarrow X$ is proper, $\Gamma_{\tilde{S}} \beta$ accumulates on a compact subset of $\tilde{S}$. This implies $\xi \in \Lambda_{\mathrm{r}}\left(\Gamma_{\tilde{S}}\right)$. The other direction for the inclusion is clear.

In [4, Bowditch proved the equivalence of several definitions of geometrically finite hyperbolic groups. In particular, we have:

Theorem 4.6 ([2], 4], 21]). $\Gamma$ is geometrically finite if and only if $\Lambda(\Gamma)=\Lambda_{\mathrm{r}}(\Gamma) \cup$ $\Lambda_{\mathrm{bp}}(\Gamma)$.

Hence, for geometrically finite $\Gamma$, we have $\Lambda_{\mathrm{p}}(\Gamma)=\Lambda_{\mathrm{bp}}(\Gamma)$.

Theorem 4.7. If $\Gamma$ is geometrically finite, then $\Gamma_{\tilde{S}}$ is geometrically finite.

Proof. Since $\Lambda(\Gamma)=\Lambda_{\mathrm{r}}(\Gamma) \cup \Lambda_{\mathrm{bp}}(\Gamma)$, it follows from Proposition 4.4 and Lemma 4.5 that $\Lambda\left(\Gamma_{\tilde{S}}\right)=\Lambda_{\mathrm{bp}}\left(\Gamma_{\tilde{S}}\right) \cup \Lambda_{\mathrm{r}}\left(\Gamma_{\tilde{S}}\right)$, proving the claim by Theorem 4.6.

\subsection{Compactness of $\operatorname{supp}\left(\mu_{E}^{\mathrm{PS}}\right)$ for horospherical $E$.}

Theorem 4.8 (Dal'bo 7]). Let $\Gamma$ be geometrically finite. For a horosphere $\mathcal{H}$ in $\mathrm{T}^{1}\left(\mathbb{H}^{n}\right)$ based at $\xi \in \partial \mathbb{H}^{n}, E:=\mathbf{p}(\mathcal{H})$ is closed in $\mathrm{T}^{1}(X)$ if and only if either $\xi \notin \Lambda(\Gamma)$ or $\xi \in \Lambda_{\mathrm{p}}(\Gamma)$.

Theorem 4.9. Let $\Gamma$ be geometrically finite. If $E:=\mathbf{p}(\mathcal{H})$ is a closed horosphere in $\mathrm{T}^{1}(X)$, then $\operatorname{supp}\left(\mu_{E}^{\mathrm{PS}}\right)$ is compact.

Proof. Let $\xi \in \partial \mathbb{H}^{n}$ be the base point for $\mathcal{H}$. The restriction of the visual map Vis : $v \mapsto v^{+}$induces a homeomorphism $\psi: \mathcal{H} \rightarrow \partial \mathbb{H}^{n} \backslash\{\xi\}$. As $E$ is closed, by Theorem 4.8 either $\xi \notin \Lambda(\Gamma)$ or $\xi$ is a bounded parabolic fixed point. If $\xi \notin \Lambda(\Gamma)$, then $\Lambda(\Gamma)$ is a compact subset of $\partial \mathbb{H}^{n} \backslash\{\xi\}$. Since $\operatorname{supp}\left(\mu_{E}^{\mathrm{PS}}\right)=\mathbf{p}\left(\psi^{-1}(\Lambda(\Gamma))\right)$, it follows that $\operatorname{supp}\left(\mu_{E}^{\mathrm{PS}}\right)$ is compact.

Suppose now that $\xi$ is a bounded parabolic fixed point. By Definition 4.2, $\Gamma_{\xi} \backslash(\Lambda(\Gamma) \backslash\{\xi\})$ is compact. Since $\Gamma_{\xi}$ is discrete, it preserves the horosphere $\mathcal{H}$ based at $\xi$, and $\Gamma_{\xi}=\Gamma_{\mathcal{H}}$. Therefore $\psi$ induces a homeomorphism between $\Gamma_{\mathcal{H}} \backslash \mathcal{H}$ and $\Gamma_{\mathcal{H}} \backslash\left(\partial \mathbb{H}^{n} \backslash\{\xi\}\right)$. It follows that $\Gamma_{\mathcal{H}} \backslash \psi^{-1}(\Lambda(\Gamma) \backslash\{\xi\})$ is compact and is equal to $\operatorname{supp}\left(\mu_{E}^{\mathrm{PS}}\right)$. 


\section{ON The CUSPIDAL NEIGHBORHOOds OF $\Lambda_{\mathrm{bp}}(\Gamma) \cap \partial \tilde{S}$}

5.1. Throughout this section, let $\Gamma$ be a torsion-free discrete subgroup of $G$ and $\tilde{S}$ a connected complete totally geodesic subspace of $\mathbb{H}^{n}$ such that the natural projection $\Gamma_{\tilde{S}} \backslash \tilde{S} \rightarrow \Gamma \backslash \mathbb{H}^{n}$ is a proper map.

The Dirichlet domain for $\Gamma_{\tilde{S}}$ attached to some $a \in \tilde{S}$ is defined by

$$
\mathcal{D}\left(a, \Gamma_{\tilde{S}}\right):=\left\{s \in \tilde{S}: d(s, a) \leq d(s, \gamma a) \text { for all } \gamma \in \Gamma_{\tilde{S}}\right\}
$$

Proposition 5.1. $\Lambda_{\mathrm{r}}(\Gamma) \cap \partial \mathcal{D}\left(a, \Gamma_{\tilde{S}}\right)=\emptyset$.

Proof. Let $\xi \in \Lambda_{r}(\Gamma) \cap \partial \mathcal{D}\left(a, \Gamma_{\tilde{S}}\right)$. As

$$
\overline{\mathcal{D}\left(a, \Gamma_{\tilde{S}}\right)}=\mathcal{D}\left(a, \Gamma_{\tilde{S}}\right) \cup\left(\partial \mathcal{D}\left(a, \Gamma_{\tilde{S}}\right) \cap \partial \mathbb{H}^{n}\right)
$$

is convex in $\mathbb{H}^{n}$, there exists a geodesic $\left\{\xi_{t}\right\} \subset \mathcal{D}\left(a, \Gamma_{\tilde{S}}\right)$ such that $\xi_{0}=a$ and $\xi_{\infty}=\xi$. As $\xi \in \Lambda_{r}\left(\Gamma_{\tilde{S}}\right)$ by Lemma 4.5, there exist sequences $t_{i} \rightarrow \infty$ and $\gamma_{i} \in \Gamma_{\tilde{S}}$ such that $d\left(\gamma_{i} \xi_{t_{i}}, a\right)$ is uniformly bounded for all $i$. Since $d\left(\xi_{t_{i}}, a\right) \rightarrow \infty$, it follows that for all large $i, d\left(\xi_{t_{i}}, \gamma_{i}^{-1} a\right)<d\left(\xi_{t_{i}}, a\right)$, yielding the fact that $\xi_{t_{i}} \notin \mathcal{D}\left(a, \Gamma_{\tilde{S}}\right)$, a contradiction.

Let $\tilde{E} \subset \mathrm{T}^{1}\left(\mathbb{H}^{n}\right)$ denote the set of all normal vectors to $\tilde{S}$. Given $U \subset \partial \mathbb{H}^{n}$, we define

$$
\mathcal{E}_{U}=\left\{v \in \tilde{E}: \pi(v) \in \mathcal{D}\left(a, \Gamma_{\tilde{S}}\right), v^{+} \in U \cap \Lambda(\Gamma)\right\} .
$$

Remark 5.2. If $\xi \in \Lambda_{r}(\Gamma) \cap \partial \tilde{S}$, then there exists a neighborhood $U$ of $\xi$ in $\partial \mathbb{H}^{n}$ such that $\mathcal{E}_{U}=\emptyset$. To see this, note that if there exists a sequence $\left\{v_{i}\right\} \subset \tilde{E}$ such that $v_{i}^{+} \rightarrow \xi$, then $\pi\left(v_{i}\right) \rightarrow \xi$, and hence by Proposition $5.1 \pi\left(v_{i}\right) \notin \mathcal{D}\left(a, \Gamma_{\tilde{S}}\right)$ for all large $i$.

In view of Theorem 4.6 and Remark [5.2 the main goal of this section is to describe the structure of $\mathcal{E}_{U}$ for a neighborhood $U$ of a point in $\Lambda_{\mathrm{bp}}(\Gamma) \cap \partial \tilde{S}$ and to compute the measure $\mu_{\tilde{E}}^{\mathrm{PS}}\left(\mathcal{E}_{U}\right)$.

In this section, we will use the upper half space model $\mathbb{H}^{n}=\mathbb{R}^{n-1} \times \mathbb{R}_{>0}$, and first we assume that

$$
\infty \in \partial \tilde{S} \cap \Lambda_{\mathrm{p}}(\Gamma)
$$

Here $\mathbb{R}^{n-1}$ is to be treated as an affine space until we make a choice of the origin. Hence $\tilde{S}$ is a vertical plane over the affine subspace $\partial \tilde{S} \backslash\{\infty\}$ of $\mathbb{R}^{n-1}$. For any affine subspace $F$ of $\mathbb{R}^{n-1}$, let $P_{F}: \mathbb{R}^{n-1} \rightarrow F$ denote the orthogonal projection. Let

$$
\mathrm{b}: \mathbb{R}^{n-1} \times \mathbb{R}_{>0} \rightarrow \mathbb{R}^{n-1} \text { and } \mathrm{h}: \mathbb{R}^{n-1} \times \mathbb{R}_{>0} \rightarrow \mathbb{R}_{>0}
$$

denote the natural projections.

Let $\Gamma^{\prime}=\Gamma_{\infty}^{\prime}$ be a normal abelian subgroup of $\Gamma_{\infty}$ with finite index, as in 4.1 and fix a $\Gamma^{\prime}$-minimal subspace $L$ of $\mathbb{R}^{n-1}$. Noting that $\mathrm{b}(a) \in \partial \tilde{S} \backslash\{\infty\}$, we choose $0:=P_{L}(\mathrm{~b}(a))$, the origin of $\mathbb{R}^{n-1}$. This choice of 0 makes $L$ a linear subspace. Set $W:=\{v-\mathrm{b}(a): v \in \partial \tilde{S} \backslash\{\infty\}\}$, a linear subspace of $\mathbb{R}^{n-1}$, and $\Delta:=\Gamma_{\tilde{S}} \cap \Gamma^{\prime}$. By Proposition 4.3, $L_{0}:=L \cap W$ is a $\Delta$-minimal (linear) subspace.

Let $V$ be the largest affine subspace of $\mathbb{R}^{n-1}$ such that $\Delta$ acts by translations on $V$. Then $0 \in L \subset V$ and $V$ is the union of all (parallel) $\Delta$-minimal subspaces of 
$\mathbb{R}^{n-1}$. There exist group homomorphisms $\tau: \Delta \rightarrow L_{0} \subset \mathbb{R}^{n-1}$ and $\theta: \Delta \rightarrow O(n-1)$ such that for any $\gamma \in \Delta$,

$$
\gamma(x)=\theta(\gamma)(x)+\tau(\gamma), \text { for all } x \in \mathbb{R}^{n-1} .
$$

We note that $V=\left\{x \in \mathbb{R}^{n-1}: \theta(\Delta) x=x\right\}$, and $V^{\perp}$ is the sum of all non-trivial (two-dimensional) $\theta(\Delta)$-irreducible subspaces of $\mathbb{R}^{n-1}$.

Lemma 5.3. (1) $W=(W \cap V)+\left(W \cap V^{\perp}\right)$;

(2) $W^{\perp}=\left(W^{\perp} \cap V\right)+\left(W^{\perp} \cap V^{\perp}\right)$.

Proof. Put $F=\partial \tilde{S} \backslash\{\infty\}$. Then $\Delta F=F$, and there exists a $\Delta$-minimal affine subspace $L_{\tilde{S}} \subset F$. Choose $0^{\prime} \in L_{\tilde{S}} \subset F \cap V$. Since $W$ is a parallel translate of $F$ through 0 , we have $W=F-0^{\prime}$. As in the proof of Proposition 4.3. $\Delta(W)=W$. Since $0 \in W$, we have $\theta(\Delta)(W)=W$, and hence $\theta(\Delta)\left(W^{\perp}\right)=W^{\perp}$. Thus $W \cap V$ is the set of fixed points of $\theta(\Delta)$ in $W$, and its orthocomplement in $W$ is the sum of all non-trivial $\theta(\Delta)$-irreducible subspaces of $W$ and it is the same as $W \cap V^{\perp}$. Therefore (1) follows, and (2) is proved similarly.

For any $v \in \tilde{E}, \pi(v) \in \tilde{S}$. By abuse of notation, we write $\mathrm{b}(v):=\mathrm{b}(\pi(v)) \in$ $\partial \tilde{S} \backslash\{\infty\}$ and $\mathrm{h}(v):=\mathrm{h}(\pi(v)) \in \mathbb{R}_{>0}$. We denote by $\sigma(v) \in W^{\perp}$ the unique element in $W^{\perp}$ of norm one satisfying

$$
v^{+}:=\operatorname{Viz}(v)=\mathrm{b}(v)+\mathrm{h}(v) \sigma(v) .
$$

Bounded parabolic assumption. For the rest of this section we will further assume that $\infty \in \partial(\tilde{S}) \cap \Lambda_{\mathrm{bp}}(\Gamma)$. Hence there exists $R_{0}>0$ such that for all $x \in \Lambda(\Gamma) \cap \mathbb{R}^{n-1}$,

$$
\left\|P_{L^{\perp}}(x)\right\| \leq R_{0}
$$

where $\|\cdot\|$ denotes the Euclidean norm.

Lemma 5.4. For any $v \in \tilde{E}$ with $v^{+} \in \Lambda(\Gamma)$,

$$
\left\|P_{V^{\perp}}(\mathrm{b}(v))\right\| \leq R_{0} .
$$

Proof. Let $0^{\prime} \in V$ be as in the proof of Lemma 5.3. Since $\mathrm{b}(v)-0^{\prime} \in W$ and $0^{\prime} \in V$, we have $P_{V^{\perp}}\left(0^{\prime}\right)=0$, and by Lemma 5.3 .

$$
P_{V^{\perp}}(\mathrm{b}(v))=P_{V^{\perp}}\left(\mathrm{b}(v)-0^{\prime}\right) \in W \text { and } P_{V^{\perp}}(\sigma(v)) \in W^{\perp} .
$$

Therefore by (5.5),$\left\|P_{V^{\perp}}(\mathrm{b}(v))\right\| \leq\left\|P_{V^{\perp}}\left(v^{+}\right)\right\|$. Since $L \subset V$, we have $V^{\perp} \subset L^{\perp}$, and hence by (5.6), $\left\|P_{V^{\perp}}\left(v^{+}\right)\right\| \leq\left\|P_{L^{\perp}}\left(v^{+}\right)\right\| \leq R_{0}$.

Proposition 5.5. There exists $R_{1}>0$ such that for all $v \in \mathcal{E}_{\partial \mathbb{H}^{n}}$,

$$
\left\|P_{L_{0}}\left(v^{+}\right)\right\| \leq R_{1} \text {. }
$$

Proof. Let $v \in \mathcal{E}_{\partial \mathbb{H}^{n}}$. Then for all $\gamma \in \Delta \subset \Gamma_{\tilde{S}}$,

$$
d_{\text {hyp }}(\pi(v), a) \leq d_{\text {hyp }}(\gamma \pi(v), a) \Rightarrow d_{\text {eucl }}(\mathrm{b}(v), \mathrm{b}(a)) \leq d_{\text {eucl }}(\gamma \mathrm{b}(v), \mathrm{b}(a)) .
$$

Now $\mathrm{b}(v)-\mathrm{b}(a) \in W, L_{0} \subset W \cap V$, and $P_{L_{0}}(\mathrm{~b}(a))=0$. As

$$
W=\left(V^{\perp} \cap W\right)+L_{0}+\left(W \cap V \cap L_{0}^{\perp}\right),
$$

which is a sum of $\theta(\Delta)$-invariant orthogonal subspaces of $W$, we get

$$
\begin{aligned}
\gamma \mathrm{b}(v)-\mathrm{b}(a)=\left[\theta(\gamma) P_{V^{\perp}}(\mathrm{b}(v))\right. & \left.-P_{V^{\perp}}(\mathrm{b}(a))\right] \\
& +\left[P_{L_{0}}(\mathrm{~b}(v))+\tau(\gamma)\right]+P_{V \cap W \cap L_{0}^{\perp}}(\mathrm{b}(v)-\mathrm{b}(a)) .
\end{aligned}
$$


Comparing this with (5.7), for any $\gamma \in \Delta$ we get

$$
\left\|P_{L_{0}}(\mathrm{~b}(v))\right\|^{2} \leq\left\|\theta(\gamma) P_{V^{\perp}}(\mathrm{b}(v))-P_{V^{\perp}}(\mathrm{b}(a))\right\|^{2}+\left\|P_{L_{0}}(\mathrm{~b}(v))+\tau(\gamma)\right\|^{2} .
$$

Since $\tau(\Delta)$ is a lattice in $L_{0}$, the radius of the smallest ball containing a fundamental domain of $\tau(\Delta)$ in $L_{0}$ is finite, which we denote by $R_{2}$. Then by (5.4) and (5.8), we conclude that

$$
\left\|P_{L_{0}}\left(v^{+}\right)\right\|^{2}=\left\|P_{L_{0}}(\mathrm{~b}(v))\right\|^{2} \leq\left(R_{0}+\|\mathrm{b}(a)\|\right)^{2}+R_{2}^{2} .
$$

By setting $R_{1}=\left(\left(R_{0}+\|\mathrm{b}(a)\|\right)^{2}+R_{2}^{2}\right)^{1 / 2}$, we finish the proof.

\subsection{Corank at $\infty$ and the structure of $\mathcal{E}_{U}$. Set}

$$
r_{\infty}:=\operatorname{rank}\left(\Gamma_{\infty}\right)-\operatorname{rank}\left(\Gamma_{\infty} \cap \Gamma_{\tilde{S}}\right) .
$$

More precisely, $r_{\infty}=\operatorname{rank}\left(\Gamma^{\prime}\right)-\operatorname{rank}(\Delta)=\operatorname{dim}(L)-\operatorname{dim}\left(L_{0}\right)$.

Proposition 5.6. If $r_{\infty}=0$, then there exists a neighborhood $U$ of $\infty$ in $\partial \mathbb{H}^{n}$ such that $\mathcal{E}_{U}=\emptyset$, where $\mathcal{E}_{U}$ is defined in (5.2).

Proof. As $r_{\infty}=0$, we have $L=L_{0}$. Therefore, for all $x \in \Lambda(\Gamma) \cap \mathbb{R}^{n-1}$,

$$
\left\|P_{L_{0}^{\perp}}(x)\right\| \leq R_{0} .
$$

Hence for any $v \in \mathcal{E}_{\partial \mathbb{H}^{n}}$, by Proposition 5.5 ,

$$
\left\|v^{+}\right\|^{2}=\left\|P_{L_{0}}\left(v^{+}\right)\right\|^{2}+\left\|P_{L_{0}^{\perp}}\left(v^{+}\right)\right\|^{2} \leq R_{1}^{2}+R_{0}^{2} .
$$

Let $U=\left\{x \in \mathbb{R}^{n-1}:\|x\|^{2}>R_{0}^{2}+R_{1}^{2}\right\} \cup\{\infty\}$. Then $\mathcal{E}_{U}=\emptyset$.

In the rest of this section, we now consider the case when

$$
r:=r_{\infty} \geq 1 \text {. }
$$

Notation 5.7. For any $\boldsymbol{s}=\left(s_{1}, \ldots, s_{r}\right) \in \mathbb{R}^{r}$ and an ordered $r$-tuple $\left(w_{1}, \ldots, w_{r}\right)$ of vectors in $\mathbb{R}^{n-1}$, we set $\boldsymbol{s} \cdot \boldsymbol{w}:=s_{1} w_{1}+\cdots+s_{r} w_{r} \in \mathbb{R}^{n-1}, \mathbb{R}^{r} \boldsymbol{w}:=\left\{\boldsymbol{s} \cdot \boldsymbol{w}: \boldsymbol{s} \in \mathbb{R}^{r}\right\}$ and $|\boldsymbol{s}|=\max \left(\left|s_{1}\right|, \ldots,\left|s_{r}\right|\right)$. For $\boldsymbol{k} \in \mathbb{Z}^{r}$ and an ordered $r$-tuple $\gamma=\left(\gamma_{1}, \ldots, \gamma_{r}\right)$ of elements of $G$, we write $\gamma^{k}=\gamma_{1}^{k_{1}} \cdots \gamma_{r}^{k_{r}} \in G$.

Fix an ordered $r$-tuple $\gamma=\left(\gamma_{1}, \ldots, \gamma_{r}\right)$ of elements of $\Gamma^{\prime}=\Gamma_{\infty}^{\prime}$ such that the subgroup generated by $\gamma \cup \Delta$ is of finite index in $\Gamma^{\prime}$. For each $\gamma_{i}$, there exists $w_{i} \in L$ and $\sigma_{i} \in O(n-1)$ such that for all $x \in \mathbb{R}^{n-1}$,

$$
\gamma_{i}(x)=\sigma_{i}(x)+w_{i} .
$$

Moreover, $\sigma_{i}$ and the translation by $w_{i}$ commutes, and hence for any $k \in \mathbb{Z}, \gamma_{i}^{k}(x)=$ $\sigma_{i}^{k}(x)+k w_{i}$.

Setting $\boldsymbol{w}=\left(w_{1}, \ldots, w_{r}\right)$ and $\sigma=\left(\sigma_{1}, \ldots, \sigma_{r}\right)$, we have that for any $x=y+z \in$ $\mathbb{R}^{n-1}$ with $y \in L^{\perp}$ and $z \in L$ and $\boldsymbol{k}=\left(k_{1}, \ldots, k_{r}\right) \in \mathbb{Z}^{r}$,

$$
\gamma^{\boldsymbol{k}}(x)=\sigma^{\boldsymbol{k}}(y)+z+\boldsymbol{k} \cdot \boldsymbol{w} .
$$

Let $R_{0}$ and $R_{1}$ be as in (5.6) and in Proposition 5.5. respectively. Set

$$
B_{0}:=\left\{x \in L^{\perp}:\|x\| \leq R_{0}\right\} \quad \text { and } \quad B_{1}:=\left\{x \in L_{0}:\|x\| \leq R_{1}\right\} .
$$

Let $M_{1}:=L \cap L_{0}^{\perp}$. Then $\mathbb{Z}^{r} \cdot P_{M_{1}}(\boldsymbol{w})$ is a lattice in $M_{1}=\mathbb{R}^{r} P_{M_{1}}(\boldsymbol{w})$, which admits a relatively compact fundamental domain, say $F_{1}$. Let $F_{2}$ be a relatively compact fundamental domain for the lattice $\tau(\Delta)$ in $L_{0}$. We define the following relatively compact subset of $\mathbb{R}^{n-1}$ :

$$
\mathcal{F}:=B_{0}+\left(B_{1}+F_{2}\right)+F_{1} \subset L^{\perp}+L_{0}+\left(L \cap L_{0}^{\perp}\right) .
$$


By (5.9),

$$
\gamma^{k} \mathcal{F}=\mathcal{F}+\boldsymbol{k} \cdot \boldsymbol{w}
$$

For related variable quantities $x \geq 0$ and $y \geq 0$, the symbol $x \gg y$ means that there exists a constant $C>0$ such that for all related $x$ and $y, x \geq C y$, and the symbol $x \asymp y$ means that $x \gg y$ and $y \gg x$.

Proposition 5.8. There exists $c_{0} \geq 1$ such that for all sufficiently large $N \geq 1$,

$$
\operatorname{Viz}\left(\mathcal{E}_{U_{c_{0} N}}\right) \subset \bigcup_{|\boldsymbol{k}| \geq N} \Delta \gamma^{\boldsymbol{k}}(\mathcal{F}),
$$

where $U_{c_{0} N}=\left\{x \in \mathbb{R}^{n-1}:\|x\| \geq c_{0} N\right\}$.

Proof. Since $\mathbb{R}^{n-1}=L^{\perp}+L_{0}+M_{1}$ for $M_{1}=L_{0}^{\perp} \cap L$, we have for any $v \in \mathbb{R}^{n-1}$,

$$
v^{+}=P_{L^{\perp}}\left(v^{+}\right)+P_{L_{0}}\left(v^{+}\right)+P_{M_{1}}\left(v^{+}\right) .
$$

Let $v \in \mathcal{E}_{\partial \mathbb{H}^{n}}$. By (5.6) and Proposition 5.5,

$$
P_{L^{\perp}}\left(v^{+}\right) \in B_{0} \text { and } P_{L_{0}}\left(v^{+}\right) \in B_{1} .
$$

In order to control $P_{M_{1}}\left(v^{+}\right)$, let $\boldsymbol{k}=\boldsymbol{k}\left(v^{+}\right) \in \mathbb{Z}^{r}$ be such that $P_{M_{1}}\left(v^{+}\right) \in$ $\boldsymbol{k} \cdot P_{M_{1}}(\boldsymbol{w})+F_{1}$, where $\boldsymbol{k}$ is uniquely determined. Let $\lambda_{\boldsymbol{k}} \in \Delta$ be such that

$$
P_{L_{0}}(\boldsymbol{k} \cdot \boldsymbol{w}) \in \tau\left(\lambda_{\boldsymbol{k}}\right)+F_{2} .
$$

Since $\boldsymbol{k} \cdot P_{M_{1}}(\boldsymbol{w})-\boldsymbol{k} \cdot \boldsymbol{w}=P_{L_{0}}(\boldsymbol{k} \cdot \boldsymbol{w})$,

$$
P_{M_{1}}\left(v^{+}\right) \in\left(\boldsymbol{k} \cdot P_{M_{1}}(\boldsymbol{w})-\boldsymbol{k} \cdot \boldsymbol{w}\right)+\left(F_{1}+\boldsymbol{k} \cdot \boldsymbol{w}\right) \in \tau\left(\lambda_{\boldsymbol{k}}\right)+F_{2}+\left(F_{1}+\boldsymbol{k} \cdot \boldsymbol{w}\right) .
$$

Therefore by (5.12), for $\boldsymbol{k}=\boldsymbol{k}\left(v^{+}\right)$, we have

$$
v^{+} \in \mathcal{F}+\boldsymbol{k} \cdot \boldsymbol{w}+\tau\left(\lambda_{\boldsymbol{k}}\right)=\lambda_{\boldsymbol{k}} \gamma^{\boldsymbol{k}}(\mathcal{F}) .
$$

Since $P_{M_{1}}: \mathbb{R}^{r} \boldsymbol{w} \rightarrow M_{1}$ is a linear isomorphism, there exists $N_{1} \geq 1$ such that for all $\boldsymbol{k} \in \mathbb{Z}^{r}$ with $|\boldsymbol{k}|>N_{1}$,

$$
\left\|P_{M_{1}}(\boldsymbol{k} \cdot \boldsymbol{w})\right\| \asymp|\boldsymbol{k}| .
$$

By (5.12) and (5.13), $\left\|P_{M_{1}}\left(v^{+}\right)-v^{+}\right\| \leq R_{0}+R_{1}$ and $P_{M_{1}}\left(v^{+}\right)-P_{M_{1}}(\boldsymbol{k} \cdot \boldsymbol{w}) \in F_{1}$ for $\boldsymbol{k}=\boldsymbol{k}\left(v^{+}\right)$. It follows that there exists a constant $B>0$ such that for all $v \in \mathcal{E}_{\partial \mathbb{H}^{n}}$,

$$
\left\|P_{M_{1}}(\boldsymbol{k} \cdot \boldsymbol{w})\right\|-B \leq\left\|v^{+}\right\| \leq\left\|P_{M_{1}}(\boldsymbol{k} \cdot \boldsymbol{w})\right\|+B,
$$

where $\boldsymbol{k}=\boldsymbol{k}\left(v^{+}\right)$. Hence by (5.15), there exists $N_{2} \geq 1$ such that for all $v \in \mathcal{E}_{\partial \mathbb{H}^{n}}$ with $\left|\boldsymbol{k}\left(v^{+}\right)\right|>N_{2}$,

$$
\left\|v^{+}\right\| \asymp\left|\boldsymbol{k}\left(v^{+}\right)\right| .
$$

In view of (5.14), this finishes the proof.

Lemma 5.9. There exists $N_{0} \geq 1$ such that for all $\boldsymbol{k} \in \mathbb{Z}^{r}$ with $|\boldsymbol{k}|>N_{0}$, the following hold:

(1) For $\xi \in \gamma^{\boldsymbol{k}}(\mathcal{F}),\|\xi\| \asymp|\boldsymbol{k}|$.

(2) For $v \in \tilde{E}$ with $v^{+} \in \boldsymbol{\gamma}^{\boldsymbol{k}}(\mathcal{F}), \mathrm{h}(v) \asymp|\boldsymbol{k}|$. 
Proof. If $\xi \in \gamma^{\boldsymbol{k}} \mathcal{F}$, then $\|\xi-\boldsymbol{k} \cdot \boldsymbol{w}\| \leq \operatorname{diam}_{\text {eucl }}(\mathcal{F})$. Hence $\|\xi\| \asymp\|\boldsymbol{k} \cdot \boldsymbol{w}\| \asymp|\boldsymbol{k}|$, proving (1).

For $v \in \tilde{E}$ such that $v^{+} \in \gamma^{k}(\mathcal{F})$, by (5.5),

$$
\mathrm{h}(v) \asymp\left\|P_{W^{\perp}}\left(v^{+}\right)\right\| \asymp P_{W^{\perp}}(\boldsymbol{k} \cdot \boldsymbol{w}) .
$$

Since $W \cap L=L_{0}$ and $L=L_{0} \oplus \mathbb{R}^{r} \boldsymbol{w}$, the map $P_{W^{\perp}}: \mathbb{R}^{r} \boldsymbol{w} \rightarrow W^{\perp}$ is injective. Therefore

from which (2) follows.

$$
\left\|P_{W \perp}(\boldsymbol{k} \cdot \boldsymbol{w})\right\| \asymp\|\boldsymbol{k} \cdot \boldsymbol{w}\| \asymp|\boldsymbol{k}|
$$

Let $o=(0,1) \in \mathbb{R}^{n-1} \times \mathbb{R}_{>0}$. For $T \geq 1$, put

$$
\mathcal{B}_{T}=\left\{v \in \tilde{E}: \beta_{\infty}(o, \pi(v)) \geq \log T\right\} ;
$$

that is, $\mathcal{B}_{T}$ is the intersection with $\tilde{E}$ of a horoball based at $\infty$. We note that for $v \in \tilde{E}, \beta_{\infty}(o, \pi(v))=\log h(v)$. Hence in the vertical plane model of $\tilde{S}, \mathcal{B}_{T}$ consists of vectors $v \in \tilde{E}$ whose base points have the Euclidean height at least $T$.

Proposition 5.10. Let $\mathcal{F}_{0}:=B_{0}+F_{2}+F_{1}$. Then $\nu_{o}\left(\mathcal{F}_{0}\right)>0$, and for all sufficiently large $T$, there exists $N \asymp T$ such that

$$
\operatorname{Viz}\left(\mathcal{B}_{T}\right) \supset \bigcup_{|\boldsymbol{k}| \geq N} \gamma^{k}\left(\mathcal{F}_{0}\right) .
$$

Proof. Since $\Delta F_{2}=L_{0}$ and $\gamma^{\mathbb{Z}^{r}} F_{1}=M_{1}$,

$$
\Delta\left(\bigcup_{\boldsymbol{k} \in \mathbb{Z}^{r}} \gamma^{\boldsymbol{k}}\left(\mathcal{F}_{0}\right)\right)=B_{0}+L_{0}+M_{1}=B_{0}+L \supset \Lambda(\Gamma) \backslash\{\infty\} .
$$

Therefore, if $\nu_{o}\left(\mathcal{F}_{0}\right)=0$, then by the conformality, it follows that $\nu_{o}(\Lambda(\Gamma) \backslash\{\infty\})=$ 0 . Since $\Gamma$ does not fix $\infty$, by the $\Gamma$-invariance of $\left\{\nu_{x}\right\}$ we get $\nu_{o}(\Lambda(\Gamma))=0$, which is a contradiction, proving the first claim.

If $v \in \tilde{E}$ and $v^{+} \in \boldsymbol{\gamma}^{\boldsymbol{k}}\left(\mathcal{F}_{0}\right)$, then by Lemma 5.9, $h(v) \asymp|\boldsymbol{k}|$. If $h(v)>T$, then $v \in \mathcal{B}_{T}$. Therefore (5.17) holds for suitable $N \asymp T$.

5.3. Estimation of $\mu_{\tilde{E}}^{\mathrm{PS}}\left(\mathcal{E}_{U}\right)$. Let $\mathrm{V}^{-1}: \mathbb{R}^{n-1} \backslash \partial \tilde{S} \rightarrow \tilde{E}$ be the inverse of the restriction of the visual map Viz $: \tilde{E} \rightarrow \partial \mathbb{H}^{n} \backslash \partial \tilde{S}=\mathbb{R}^{n-1} \backslash \partial \tilde{S}$.

Lemma 5.11. There exists $N_{1} \geq 1$ such that for all $\boldsymbol{k} \in \mathbb{Z}^{r}$ with $|\boldsymbol{k}|>N_{1}$,

$$
\int_{\xi \in \boldsymbol{\gamma}^{k} \mathcal{F}} e^{\delta \beta_{\xi}\left(o, \pi\left(\mathrm{V}^{-1}(\xi)\right)\right)} d \nu_{o}(\xi) \asymp|\boldsymbol{k}|^{-\delta} .
$$

Proof. We have $\left\|P_{W^{\perp}}(\boldsymbol{k} \cdot \boldsymbol{w})\right\| \asymp|\boldsymbol{k}|$. Hence for sufficiently large $|\boldsymbol{k}|$, we have that $\gamma^{k} \mathcal{F} \cap \partial \tilde{S}=\emptyset$. Note that the Euclidean diameter of the horosphere based at $\xi$ and passing through $o=(0,1) \in \mathbb{R}^{n-1} \times \mathbb{R}_{>0}$ is $1+\|\xi\|^{2}$, and the diameter of the horosphere based at $\xi$ and passing through $\pi\left(\mathrm{V}^{-1}(\xi)\right)$ is $\mathrm{h}\left(\pi\left(\mathrm{V}^{-1}(\xi)\right)\right)$. Therefore the signed hyperbolic distance of the segment cut by these two horospheres on the vertical geodesic ending in $\xi$ is

$$
\beta_{\xi}\left(o, \pi\left(\mathrm{V}^{-1}(\xi)\right)\right)=\log \left(1+\|\xi\|^{2}\right)-\log \left(\mathrm{h}\left(\pi\left(\mathrm{V}^{-1}(\xi)\right)\right)\right) .
$$

Hence by Lemma 5.9 ,

$$
e^{\delta \beta_{\xi}\left(o, \pi\left(\mathrm{V}^{-1}(\xi)\right)\right)}=\left(\frac{1+\|\xi\|^{2}}{\mathrm{~h}\left(\pi\left(\mathrm{V}^{-1}(\xi)\right)\right)}\right)^{\delta} \asymp|\boldsymbol{k}|^{\delta} .
$$


By conformality and $\Gamma$-invariance of Patterson-Sullivan density $\left\{\nu_{x}\right\}$,

$$
\begin{aligned}
\nu_{o}\left(\boldsymbol{\gamma}^{\boldsymbol{k}} \mathcal{F}\right)=\left(\boldsymbol{\gamma}^{-\boldsymbol{k}} \nu_{o}\right)(\mathcal{F})=\nu_{\boldsymbol{\gamma}^{-k} \cdot o}(\mathcal{F}) & =\int_{\xi \in \mathcal{F}} \frac{d \nu_{\boldsymbol{\gamma}^{-\boldsymbol{k}_{o}}}}{d \nu_{o}}(\xi) d \nu_{o}(\xi) \\
& =\int_{\xi \in \mathcal{F}} e^{-\delta \beta_{\xi}\left(\boldsymbol{\gamma}^{-k}, o\right)} d \nu_{o}(\xi) .
\end{aligned}
$$

We note that the horosphere based at $\xi$ passing through $\gamma^{-\boldsymbol{k}_{O}}=(-\boldsymbol{k} \cdot \boldsymbol{w}, 1) \in$ $\mathbb{R}^{n-1} \times \mathbb{R}_{>0}$ has diameter $1+\|\xi+\boldsymbol{k} \cdot \boldsymbol{w}\|^{2}$. Therefore

$$
\beta_{\xi}\left(\boldsymbol{\gamma}^{-\boldsymbol{k}} o, o\right)=\log \left(1+\|\xi+\boldsymbol{k} \cdot \boldsymbol{w}\|^{2}\right)-\log \left(1+\|\xi\|^{2}\right),
$$

and hence, since $\|\boldsymbol{k} \cdot \boldsymbol{w}\| \asymp|\boldsymbol{k}|$ for all large $|\boldsymbol{k}|$, we have, for any $\xi \in \mathcal{F}$,

$$
e^{-\delta \beta_{\xi}\left(\gamma^{\boldsymbol{k}} o, o\right)}=\left(\frac{1+\|\boldsymbol{k} \cdot \boldsymbol{w}-\xi\|^{2}}{1+\|\xi\|^{2}}\right)^{-\delta} \asymp|\boldsymbol{k}|^{-2 \delta} .
$$

Since $\nu_{o}(\mathcal{F})>0$ by Proposition 5.10, we deduce from (5.19) that

$$
\nu_{o}\left(\gamma^{\boldsymbol{k}} \mathcal{F}\right) \asymp|\boldsymbol{k}|^{-2 \delta} \nu_{o}(\mathcal{F}) \asymp|\boldsymbol{k}|^{-2 \delta} .
$$

Together with (5.18), this proves the claim.

Let $\mathbf{p}: \tilde{E} \rightarrow \Gamma_{\tilde{E}} \backslash \tilde{E}$ be the natural quotient map. We note that $\Gamma_{\tilde{E}}=\Gamma_{\tilde{S}}$. From 2.6. we recall that the measure $\mu_{\tilde{E}}^{\mathrm{PS}}$, which is $\Gamma_{\tilde{E}}$-invariant, naturally induces a measure on $\Gamma_{\tilde{E}} \backslash \tilde{E}$. The pushforward of this measure from $\Gamma_{\tilde{E}} \backslash \tilde{E}$ to $E=\mathbf{p}(\tilde{E})$ is $\mu_{E}^{\mathrm{PS}}$.

Recall the definition of $c_{0}>0$ and $U_{c_{0} N}$ from Proposition 5.8

Proposition 5.12. (1) For all sufficiently large $N \geq 1$, we have

$$
\mu_{E}^{\mathrm{PS}}\left(\mathbf{p}\left(\mathcal{E}_{U_{c_{0} N}}\right)\right) \ll \sum_{\boldsymbol{k} \in \mathbb{Z}^{r} \backslash\{0\}}|\boldsymbol{k}|^{-\delta} .
$$

(2) For all sufficiently large $T \geq 1$, we have

$$
\mu_{E}^{\mathrm{PS}}\left(\mathbf{p}\left(\mathcal{B}_{T}\right)\right) \gg \sum_{\boldsymbol{k} \in \mathbb{Z}^{r} \backslash\{0\}}|\boldsymbol{k}|^{-\delta} .
$$

Proof. By Proposition 5.8 and Lemma 5.11, for all large $N \geq 1$,

$$
\begin{aligned}
\mu_{E}^{\mathrm{PS}}\left(\mathbf{p}\left(\mathcal{E}_{U_{c_{0} N}}\right)\right) & \leq \sum_{|\boldsymbol{k}| \geq N} \mu_{\tilde{E}}^{\mathrm{PS}}\left(\mathrm{V}^{-1}\left(\boldsymbol{\gamma}^{\boldsymbol{k}}(\mathcal{F})\right)\right) \\
& =\sum_{|\boldsymbol{k}| \geq N} \int_{\xi \in \boldsymbol{\gamma}^{\boldsymbol{k}} \mathcal{F}} e^{\delta \beta_{\xi}\left(o, \pi\left(\mathrm{V}^{-1}(\xi)\right)\right)} d \nu_{o}(\xi) \\
& \ll \sum_{\boldsymbol{k} \geq N}|\boldsymbol{k}|^{-\delta},
\end{aligned}
$$

proving (1).

Consider the natural quotient map

$$
\mathbf{p}_{\infty}:\left(\Gamma_{\tilde{E}} \cap \Gamma_{\infty}\right) \backslash \tilde{E} \rightarrow \Gamma_{\tilde{E}} \backslash \tilde{E} .
$$

Since $\infty \in \Lambda_{\mathrm{bp}}(\Gamma)$, there exists $T_{0}>0$ such that $\mathbf{p}_{\infty}$ restricted to $\left(\Gamma_{\tilde{E}} \cap \Gamma_{\infty}\right) \backslash \mathcal{B}_{T}$ is proper and injective for all $T \geq T_{0}$.

Now since $F_{2}$ is a fundamental domain for $\Delta$ action on $L_{\tilde{S}}$ and $F_{1}$ is a fundamental domain for the action of $\left\{\gamma^{k}: k \in \mathbb{Z}^{r}\right\}$ on $M_{1}$, the quotient map $\tilde{E} \rightarrow \Delta \backslash \tilde{E}$ 
is injective on $\bigcup_{|\boldsymbol{k}|>N} \mathrm{~V}^{-1}\left(\gamma^{\boldsymbol{k}}\left(\mathcal{F}_{0}\right)\right)$. Since $\left[\Gamma_{\tilde{S}} \cap \Gamma_{\infty}: \Delta\right]<\infty$ and $\mathbf{p}_{\infty}$ is injective on $\left(\Gamma_{\tilde{S}} \cap \Gamma_{\infty}\right) \backslash \mathcal{B}_{T}$, for all sufficiently large $T \gg 1$,

$$
\begin{aligned}
\mu_{E}^{\mathrm{PS}}\left(\mathbf{p}\left(\mathcal{B}_{T}\right)\right) & =\mu_{\Gamma_{\tilde{E}}^{\mathrm{PS}} \backslash \tilde{E}}^{\mathrm{P}}\left(\mathbf{p}_{\infty}\left(\mathcal{B}_{T}\right)\right) ; \quad \text { see }(2.13) \\
& \gg \sum_{|\boldsymbol{k}| \geq N} \mu_{\tilde{E}}^{\mathrm{PS}}\left(\mathrm{V}^{-1}\left(\boldsymbol{\gamma}^{\boldsymbol{k}}\left(\mathcal{F}_{0}\right)\right)\right) ; \quad \text { by Proposition } 5.10 \\
& \gg \sum_{\boldsymbol{k} \geq N}|\boldsymbol{k}|^{-\delta} ; \quad \text { by Lemma (5.11). }
\end{aligned}
$$

This proves (2).

\section{PARAbolic CORANK AND CRIterion FOR Finiteness of $\mu_{E}^{\mathrm{PS}}$}

Let $\Gamma$ be non-elementary torsion-free discrete subgroup of $G$. Let $\tilde{S}, \tilde{E}$ and $E$ be as in 95 . In particular, $\tilde{S}$ is totally geodesic and the map $\Gamma_{\tilde{S}} \backslash \tilde{S} \rightarrow \Gamma \backslash \mathbb{H}^{n}$ is proper.

Definition 6.1 (Parabolic corank). Define

$$
\operatorname{pb-corank}\left(\Gamma_{\tilde{S}}\right)=\max _{\xi \in \Lambda_{\mathrm{p}}(\Gamma) \cap \partial(\tilde{S})}\left(\operatorname{rank}\left(\Gamma_{\xi}\right)-\operatorname{rank}\left(\Gamma_{\xi} \cap \Gamma_{\tilde{S}}\right)\right) .
$$

When $\Lambda_{\mathrm{p}}(\Gamma) \cap \partial(\tilde{S})=\emptyset$, we set $\operatorname{pb}-\operatorname{corank}\left(\Gamma_{\tilde{S}}\right)=0$.

Lemma 6.2 (Corank Lemma). pb-corank $\left(\Gamma_{\tilde{S}}\right) \leq \operatorname{codim}(\tilde{S})$.

Proof. Suppose $\infty \in \Lambda_{\mathrm{p}}(\Gamma) \cap \partial \tilde{S}$. Let $L$ be a $\Gamma_{\infty}^{\prime}$-minimal subspace of $\partial \mathbb{H}^{n} \backslash \infty$ and let $W$ be the intersection of a translate of $\partial \tilde{S} \backslash\{\infty\}$ through a point in L. Then by Proposition 4.3, $\operatorname{rank}\left(\Gamma_{\infty}^{\prime}\right)-\operatorname{rank}\left(\Gamma_{\tilde{S}} \cap \Gamma_{\infty}^{\prime}\right)=\operatorname{dim}(L)-\operatorname{dim}(W) \leq$ $(n-1)-\operatorname{dim}(\partial \tilde{S})=n-\operatorname{dim} \tilde{S}$.

6.1. Finiteness criterion for geometrically finite $\Gamma$. For the rest of this section we further assume that $\Gamma$ is geometrically finite.

Theorem 6.3. $\operatorname{pb}-\operatorname{corank}\left(\Gamma_{\tilde{S}}\right)=0 \Leftrightarrow \operatorname{supp}\left(\mu_{E}^{\mathrm{PS}}\right)$ is compact.

Proof. Suppose that $\operatorname{supp}\left(\mu_{E}^{\mathrm{PS}}\right)$ is not compact. Fix a Dirichlet domain $\mathcal{D}\left(a, \Gamma_{\tilde{S}}\right)$ for the $\Gamma_{\tilde{S}}$ action on $\tilde{S}$. Since the projection of $\Gamma_{\tilde{E}} \backslash \tilde{E}$ into $\Gamma \backslash \mathrm{T}^{1}\left(\mathbb{H}^{n}\right)$ is proper, there exists an unbounded sequence $v_{m} \in \tilde{E}$ with $\pi\left(v_{m}\right) \in \mathcal{D}\left(a, \Gamma_{\tilde{S}}\right)$ and $v_{m}^{+} \in \Lambda(\Gamma)$. Since $\Lambda(\Gamma)$ is compact, by passing to a subsequence, we assume that $v_{m}^{+} \rightarrow \xi$ for some $\xi \in \Lambda(\Gamma)$. Thus for any neighborhood $U$ of $\xi$ in $\partial \mathbb{H}^{n}$, we have $v_{m} \in \mathcal{E}_{U}$ for all large $m$.

Consider the upper half space model $\mathbb{H}^{n}=\mathbb{R}^{n-1} \times \mathbb{R}_{>0}$ with $\xi$ identified with $\infty$ as in \$5. As $v_{m}^{+} \rightarrow \xi=\infty$, by (5.5) we have $\left\|\mathrm{b}\left(v_{m}\right)\right\| \rightarrow \infty$ or $\mathrm{h}\left(v_{m}\right) \rightarrow \infty$ (see (5.3) for notation), and hence $\pi\left(v_{m}\right) \rightarrow \infty=\xi$. Therefore $\xi \in \partial\left(\mathcal{D}\left(a, \Gamma_{\tilde{S}}\right)\right)$. By Proposition 5.1, $\xi \notin \Lambda_{\mathrm{r}}(\Gamma)$. Since $\Gamma$ is geometrically finite, by Theorem 4.6. $\xi \in \Lambda_{\text {bp }}(\Gamma) \cap \partial \mathcal{D}\left(a, \Gamma_{\tilde{S}}\right)$. Now by Proposition [5.6, pb-corank $\left(\Gamma_{\tilde{S}}\right) \neq 0$.

To prove the converse, suppose that there exists $\xi \in \Lambda_{\mathrm{bp}}(\Gamma) \cap \partial \tilde{S}$ such that $r=\operatorname{rank}\left(\Gamma_{\xi}\right)-\operatorname{rank}\left(\Gamma_{\xi} \cap \Gamma_{\tilde{S}}\right) \geq 1$. Without loss of generality, we may assume $\xi=\infty$. Fix $T_{0}>1$. The map $\mathbf{p}_{\infty}$ as in (5.20) restricted to $\left(\Gamma_{\tilde{E}} \cap \Gamma_{\infty}\right) \backslash \mathcal{B}_{T_{0}}$ is proper (see (5.16) for notation). Therefore for any compact subset $\Omega$ of $\Gamma_{\tilde{E}} \backslash \tilde{E}$, we have $\mathbf{p}_{\infty}\left(\mathcal{B}_{T}\right) \cap \Omega=\emptyset$ for all sufficiently large $T>T_{0}$. By Proposition 5.12 (2),

$$
\mu_{E}^{\mathrm{PS}}\left(\mathbf{p}\left(\mathcal{B}_{T}\right)\right) \gg \sum_{\boldsymbol{k} \in \mathbb{Z}^{r} \backslash\{0\}}|\boldsymbol{k}|^{-\delta}>0 .
$$

Therefore $\operatorname{supp}\left(\mu_{E}^{\mathrm{PS}}\right)$ intersects $\mathbf{p}\left(\mathcal{B}_{T}\right)$ for all large $T \gg 1$. Since the projection of $\Gamma_{\tilde{E}} \backslash \tilde{E}$ into $\Gamma \backslash \mathrm{T}^{1}\left(\mathbb{H}^{n}\right)$ is proper, $\operatorname{supp}\left(\mu_{E}^{\mathrm{PS}}\right)$ is non-compact. 
Theorem 6.4. $\operatorname{pb}-\operatorname{corank}\left(\Gamma_{\tilde{S}}\right)<\delta \Leftrightarrow\left|\mu_{E}^{\mathrm{PS}}\right|<\infty$.

Proof. Suppose that pb-corank $\left(\Gamma_{\tilde{S}}\right) \geq \delta>0$. Then there exists $\xi \in \Lambda_{\mathrm{bp}}(\Gamma) \cap \tilde{S}$ such that $r:=\operatorname{rank}\left(\Gamma_{\xi}\right)-\operatorname{rank}\left(\Gamma_{\xi} \cap \Gamma_{\tilde{S}}\right) \geq \max \{\delta, 1\}$. Without loss of generality, we may assume $\xi=\infty$. By the second part of the proof of Theorem 6.3, for all sufficiently large $T \gg 1$, since $r \geq \delta$,

$$
\left|\mu_{E}^{\mathrm{PS}}\right| \geq \mu_{E}^{\mathrm{PS}}\left(\mathbf{p}\left(\mathcal{B}_{T}\right)\right) \gg \sum_{\boldsymbol{k} \in \mathbb{Z}^{r} \backslash\{0\}}|\boldsymbol{k}|^{-\delta}=\infty .
$$

Now suppose that pb-corank $\left(\Gamma_{\tilde{S}}\right)<\delta$. By the compactness of $\Lambda(\Gamma) \cap \partial\left(\mathcal{D}\left(a, \Gamma_{\tilde{S}}\right)\right)$, where $\mathcal{D}\left(a, \Gamma_{\tilde{S}}\right)$ is a fixed Dirichlet domain for $\Gamma_{\tilde{S}}$, to prove finiteness of $\mu_{E}^{\mathrm{PS}}$ it suffices to show that for every $\xi \in \Lambda(\Gamma) \cap \partial\left(\mathcal{D}\left(a, \Gamma_{\tilde{S}}\right)\right)$, there exists a neighborhood $U$ of $\xi$ in $\partial \mathbb{H}^{n}$ such that $\mu_{E}^{\mathrm{PS}}\left(\mathbf{p}\left(\mathcal{E}_{U}\right)\right)<\infty$ with $\mathcal{E}_{U}$ defined as in (5.2). By Proposition 5.1 and Theorem 4.7, $\xi \in \Lambda_{\mathrm{bp}}(\Gamma)$. Let $r:=\operatorname{rank}\left(\Gamma_{\xi}\right)-\operatorname{rank}\left(\Gamma_{\xi} \cap \Gamma_{\tilde{S}}\right)$. If $r=0$, then by Proposition 5.6. there exists a neighborhood $U$ of $\xi$ such that $\mathcal{E}_{U}=\emptyset$. Therefore we assume that $\delta>r \geq 1$. By Proposition 5.12(1), there exists a neighborhood $U$ of $\xi$ such that

$$
\mu_{E}^{\mathrm{PS}}\left(\mathbf{p}\left(\mathcal{E}_{U}\right)\right) \ll \sum_{\boldsymbol{k} \in \mathbb{Z}^{r} \backslash\{0\}}|\boldsymbol{k}|^{-\delta}<\infty .
$$

\subsection{Finiteness of $\left|\mu_{E}^{\mathrm{Leb}}\right|$ and $\left|\mu_{E}^{\mathrm{PS}}\right|$.}

Theorem 6.5. Let $\tilde{S}$ be any totally geodesic immersion in $\mathbb{H}^{n}$. Suppose that $\operatorname{dim}(\tilde{S}) \geq(n+1) / 2$ and $\left|\mu_{E}^{\mathrm{Leb}}\right|<\infty$. Then $\left|\mu_{E}^{\mathrm{PS}}\right|<\infty$.

Proof. Since $\Gamma_{\tilde{S}}$ is a lattice in $G_{\tilde{S}}, \Lambda\left(\Gamma_{\tilde{S}}\right)=\partial \tilde{S}$. Hence by Theorem 2.21, the natural map $\mathbf{p}: \Gamma_{\tilde{S}} \backslash \tilde{S} \rightarrow \Gamma \backslash \mathbb{H}^{n}$ is proper.

Let $k:=\operatorname{dim}(\tilde{S}) \geq\lceil(n+1) / 2\rceil \geq 2$. By a property of a lattice in rank one Lie group $G_{\tilde{S}}, \operatorname{rank}\left(\Gamma_{\tilde{S}} \cap \Gamma_{\xi}\right)=k-1$ (cf. [29, $\left.\left.\S 13.8\right]\right)$. Therefore by Lemma 6.2,

$$
r:=\operatorname{pb}-\operatorname{corank}\left(\Gamma_{\tilde{S}}\right) \leq n-k \leq n-(n+1) / 2 \leq(n-1) / 2 .
$$

Let $\xi \in \partial(\tilde{S}) \cap \Lambda_{\mathrm{bp}}\left(\Gamma_{\tilde{S}}\right)$ be such that $\operatorname{rank}\left(\Gamma_{\tilde{S}} \cap \Gamma_{\xi}\right)=r$. Then $\operatorname{rank}\left(\Gamma_{\xi}\right) \geq(k-1)+r$. By a result of Dalbo, Otal and Peign [8, Proposition 2],

$$
\delta>\operatorname{rank}\left(\Gamma_{\xi}\right) / 2 \geq((k-1)+r) / 2 \geq(k-1+(n-k)) / 2=(n-1) / 2 \geq r .
$$

Hence by Theorem 6.4, $\left|\mu_{E}^{\mathrm{PS}}\right|$ is finite.

As an immediate corollary, we state:

Corollary 6.6. Let $n=2,3$. Then $\left|\mu_{E}^{\mathrm{Leb}}\right|<\infty$ implies that $\left|\mu_{E}^{\mathrm{PS}}\right|<\infty$.

To deduce that $\operatorname{sk}_{\Gamma}\left(w_{0}\right)>0$, when $w_{0} \Gamma$ is infinite in Theorem 1.2 we need the following. Here $\Gamma$ need not be geometrically finite.

Proposition 6.7. If $\left[\Gamma: \Gamma_{\tilde{S}}\right]=\infty$, then $\Lambda(\Gamma) \not \subset \partial_{\infty}(\tilde{S})$, and $\left|\mu_{E}^{\mathrm{PS}}\right|>0$.

Proof. Suppose on the contrary that $\Lambda(\Gamma) \subset \partial_{\infty}(\tilde{S})$. Let $L$ be a geodesic joining two distinct points, say $\xi_{1}, \xi_{2} \in \Lambda(\Gamma)$. Then $L \subset \tilde{S}$. For any $\gamma \in \Gamma$, we have $\gamma L$ as the geodesic joining $\gamma \xi_{1}$ and $\gamma \xi_{2}$, and hence $\gamma L \subset \tilde{S}$. Now fix $x_{0} \in L$. Then $\Gamma x_{0} \subset \tilde{S}$. Since $\Gamma_{\tilde{S}} \backslash \tilde{S} \rightarrow \Gamma \backslash \mathbb{H}^{n}$ is a proper map, we get that $\Gamma_{\tilde{S}} \backslash \Gamma$ is finite, a contradiction to our assumption. 


\section{Orbital COUnting For Discrete hyperbolic Groups}

As before, let $G=\mathrm{SO}(n, 1)^{\circ}$ for $n \geq 2$ and $\Gamma$ a torsion-free, non-elementary, discrete subgroup of $G$.

7.1. Computation with $\tilde{m}^{\text {BR }}$. Let $K$ be a maximal compact subgroup of $G$. Let $o \in \mathbb{H}^{n}$ be such that $K=G_{o}$. Then $G / K \cong \mathbb{H}^{n}$. Let $X_{0} \in \mathrm{T}_{o}^{1}\left(\mathbb{H}^{n}\right)$ and $M=G_{X_{0}}$. Then $G / M \cong \mathrm{T}^{1}\left(\mathbb{H}^{n}\right)$, where $g[M]=g X_{0}$. Let $A=\left\{a_{r}: r \in \mathbb{R}\right\} \subset Z_{G}(M)$ be a one-parameter subgroup of $G$ consisting of diagonalizable elements such that $\mathcal{G}^{r}\left(X_{0}\right)=a_{r}[M]$. Via the map $k \mapsto k X_{0}^{+}$, we have $K / M \cong \partial \mathbb{H}^{n}$.

Let $N<G$ be the expanding horospherical subgroup with respect to the right $a_{r}$-action; that is,

$$
N:=\left\{g \in G: a_{r} g a_{r}^{-1} \rightarrow e \quad \text { as } r \rightarrow \infty\right\} .
$$

The $N$-leaves $g N M / M$ correspond to unstable horospheres $\mathcal{H}_{g X_{0}}^{+}$in $\mathrm{T}^{1}(G / K)=$ $G / M$ based at $g X_{0}^{-}$. The map $N \ni z \mapsto z X_{0}^{+} \in \partial \mathbb{H}^{n} \backslash\left\{X_{0}^{-}\right\}$is a diffeomorphism.

As before, let $m_{o}$ denote the $G$-invariant (Lebesgue) conformal density $\left\{m_{x}\right\}_{x \in \mathbb{H}^{n}}$ on $\partial \mathbb{H}^{n}$. We normalize it so that $m_{o}$ (and hence every $m_{x}$ ) is a probability measure. Here $m_{o}$ is $K$-invariant.

Lemma 7.1. For any $g \in G$, consider the measure $\lambda_{g}$ on $N$ given by

$$
d \lambda_{g}(z)=e^{(n-1) \beta_{g z X_{0}^{+}}(o, g z(o))} d m_{o}\left(g z X_{0}^{+}\right) \text {, where } z \in N .
$$

Then $\lambda_{g}=\lambda_{e}$. In particular, $\lambda_{e}$ is a Haar measure on $N$ which we shall denote by the integral $d n=d \lambda_{e}(n)$ on $N$

Proof. Since $\left\{m_{x}\right\}$ is a $G$-invariant conformal density,

$$
d m_{o}\left(g z X_{0}^{+}\right)=d m_{g^{-1}(o)}\left(z X_{0}^{+}\right)=e^{(n-1) \beta_{z X_{0}^{+}}\left(o, g^{-1}(o)\right)} d m_{o}\left(z X_{0}^{+}\right) .
$$

Since $\beta_{g z X_{0}^{+}}(o, g z(o))=\beta_{z X_{0}^{+}}\left(g^{-1}(o), z(o)\right)$,

$$
d \lambda_{g}(z)=e^{(n-1) \beta_{z X_{0}^{+}}(o, z(o))} d m_{o}\left(z X_{0}^{+}\right)=d \lambda_{e}(z) .
$$

For any $g \in N, d \lambda_{e}(g z)=d \lambda_{g}(z)=d \lambda_{e}(z)$. Therefore $\lambda_{e}$ is $N$-invariant.

Notation 7.2. Note that $G_{X_{0}^{-}}=A N M$ and $K \cap G_{X_{0}^{-}}=M$. For $\psi \in C(K)$ and a measure $\lambda$ on $\partial \mathbb{H}^{n}=K X_{0}^{-} \cong K / M$, we define

$$
\int_{k \in K} \psi(k) d \lambda\left(k X_{0}^{-}\right):=\int_{K / M}\left(\int_{m \in M} \psi(k m) d m\right) d \lambda(k M) .
$$

We also fix a Patterson-Sullivan density $\left\{\nu_{x}\right\}$ on $\partial \mathbb{H}^{n}$ and consider $\tilde{m}^{\text {BR }}$ defined as in 3.1 with respect to $\left\{m_{x}\right\}$ and $\left\{\nu_{x}\right\}$.

Proposition 7.3. For any $\phi \in C_{c}\left(\mathrm{~T}^{1}\left(\mathbb{H}^{n}\right)\right)=C_{c}(G)^{M}$,

$$
\tilde{m}^{\mathrm{BR}}(\phi)=\int_{k \in K} \int_{r \in \mathbb{R}} \int_{n \in N} \phi\left(k a_{r} n\right) e^{-\delta r} d n d r d \nu_{o}\left(k X_{0}^{-}\right) .
$$

Proof. By definition,

$$
\tilde{m}^{\mathrm{BR}}(\phi)=\int \phi(u) e^{(n-1) \beta_{u^{+}}(o, \pi(u))} e^{\delta \beta_{u^{-}}(o, \pi(u))} d m_{o}\left(u^{+}\right) d \nu_{o}\left(u^{-}\right) d t,
$$


where $t=\beta_{u^{-}}(o, \pi(u))$. Let $u=k a_{r} n X_{0}$. Then, since $G_{X_{0}^{-}}=M A N$, we have $u^{-}=k a_{r} n X_{0}^{-}=k X_{0}^{-}$and

$$
\begin{aligned}
t= & \beta_{u^{-}}(o, \pi(u))=\beta_{k X_{0}^{-}}\left(o, k a_{r} n o\right)=\beta_{X_{0}^{-}}\left(o, a_{r} n o\right) \\
& =\lim _{t \rightarrow \infty} d\left(o, a_{-t} o\right)-d\left(a_{r} n o, a_{-t} o\right) \\
& =\lim _{t \rightarrow \infty} t-d\left(a_{t+r} n a_{-t-r}\left(a_{t+r} o\right), o\right) \\
& =\lim _{t \rightarrow \infty} t-d\left(a_{t+r} o, o\right)=-r .
\end{aligned}
$$

Therefore $e^{\delta \beta_{u^{-}}(o, \pi(u))} d \nu_{o}\left(u^{-}\right)=e^{-\delta r} d \nu_{o}\left(k X_{0}^{-}\right)$. Also, by Lemma 7.1 for a fixed $g=k a_{r}$ and a variable $z=n \in N$,

$$
e^{(n-1) \beta_{k a_{r} n X_{0}^{+}}\left(o, k a_{r} n \pi(o)\right)} d m_{o}\left(k a_{r} n X_{0}^{+}\right)=d \lambda_{k a_{r}}(n)=d \lambda_{e}(n)=d n .
$$

Putting all of this together proves the claim.

Notation 7.4. (1) Let $d k$ denote the probability Haar measure on $K$. Since $m_{o}$ is a $K$-invariant probability measure on $\partial \mathbb{H}^{n}=K / M$, we have that $d k=d m_{o}\left(k X_{0}^{-}\right)$ (and similarly $d k=d m_{o}\left(k X^{+}\right)$). We fix the Haar measure $d g$ on $G$ given as follows: for $g=k a_{r} n \in K A N, d g=e^{-(n-1) r} d n d r d k$. Since $G$ is unimodular, $d g=d g^{-1}$. Therefore if we express $g=n a_{r} k$, then $d g=e^{(n-1) r} d n d r d k$, and if we express $g=a_{r} n k$, then $d g=d r d n d k$.

(2) For $\epsilon>0$, let $U_{\epsilon}$ denote the $\epsilon$-neighborhood of $e$ in $G$. By an approximate identity on $G$, we mean a family of non-negative continuous functions $\left\{\psi_{\epsilon}\right\}_{\epsilon>0}$ on $G$ with $\operatorname{supp}\left(\psi_{\epsilon}\right) \subset U_{\epsilon}$ and $\int_{G} \psi_{\epsilon}(g) d g=1$.

(3) For $\xi \in C(M \backslash K)$ and $\psi \in C_{c}(G)$ and a measurable $\Omega \subset K$ with $M \Omega=\Omega$, we define a function $\xi *_{\Omega} \psi \in C_{c}(G / M)$ by

$$
\xi *_{\Omega} \psi(g):=\int_{k \in \Omega} \xi(k) \psi(g k) d k .
$$

For $\psi \in C_{c}(\Gamma \backslash G)$, we define $\xi *_{\Omega} \psi \in C_{c}(\Gamma \backslash G / M)$ similarly.

Proposition 7.5. Let $\left\{\psi_{\epsilon}\right\}_{\epsilon>0}$ be an approximate identity on $G$. Let $f \in C(M \backslash K)$ and $\Omega \subset K$ be such that $M \Omega=\Omega$ and $\nu_{o}\left(\partial\left(\Omega^{-1}\right) X_{0}^{-}\right)=0$. Then

$$
\lim _{\epsilon \rightarrow 0} \tilde{m}^{\mathrm{BR}}\left(f *_{\Omega} \psi_{\epsilon}\right)=\int_{k \in \Omega^{-1}} f\left(k^{-1}\right) d \nu_{o}\left(k X_{0}^{-}\right) .
$$

Proof. Note that for some uniform constants $\ell_{1}, \ell_{2}>0$, we have for all $k \in K$ and for all small $\epsilon>0$,

$$
k^{-1} U_{\epsilon} \subset U_{\ell_{1} \epsilon} k^{-1} \subset\left(A \cap U_{\ell_{2} \epsilon}\right)\left(N \cap U_{\ell_{2} \epsilon}\right) k^{-1}\left(K \cap U_{\ell_{2} \epsilon}\right) .
$$

Set $K_{\epsilon}:=\left(K \cap U_{\ell_{2} \epsilon}\right), \Omega_{\epsilon+}=\Omega K_{\epsilon}$ and $\Omega_{\epsilon-}=\bigcap_{k \in K_{\epsilon}} \Omega k$.

In view of the decomposition $G=A N K$, for a function $\phi$ on $K$, we define a function $\mathcal{R}_{\phi}$ on $G$ by $\mathcal{R}_{\phi}(g)=\phi(k)$ for $g=$ ank $\in A N K$. For any $\eta>0$, there exists $\epsilon>0$ such that for all $k \in K$ and $g \in U_{\epsilon}$,

$$
\mathcal{R}_{f \cdot \chi_{\Omega_{\epsilon-}}}\left(k^{-1}\right)-\eta \leq \mathcal{R}_{f \cdot \chi_{\Omega}}\left(k^{-1} g\right) \leq \mathcal{R}_{f \cdot \chi_{\Omega_{\epsilon+}}}\left(k^{-1}\right)+\eta
$$


Now by Proposition 7.3 ,

$$
\begin{aligned}
& \tilde{m}^{\mathrm{BR}}\left(f *_{\Omega} \psi_{\epsilon}\right) \\
& =\int_{g \in G} \int_{k^{\prime} \in \Omega} \psi_{\epsilon}\left(g k^{\prime}\right) f\left(k^{\prime}\right) d k^{\prime} d \tilde{m}^{\mathrm{BR}}(g) \\
& =\int_{\left(k, a_{r}, n\right) \in K \times A \times N} \int_{k^{\prime} \in \Omega} \psi_{\epsilon}\left(k a_{r} n k^{\prime}\right) f\left(k^{\prime}\right) e^{-\delta r} d k^{\prime} d n d r d \nu_{o}\left(k X_{0}^{-}\right) \\
& =\int_{k \in K} \int_{\left(a_{r}, n, k^{\prime}\right) \in A \times N \times K} \psi_{\epsilon}\left(k a_{r} n k^{\prime}\right) f\left(k^{\prime}\right) \chi_{\Omega}\left(k^{\prime}\right) e^{-\delta r} d r d n d k^{\prime} d \nu_{o}\left(k X_{0}^{-}\right) \\
& =\int_{k \in K} \int_{g \in G} \psi_{\epsilon}(k g) \mathcal{R}_{f \cdot \chi_{\Omega}}(g) e^{-\delta r_{g}} d g d \nu_{o}\left(k X_{0}^{-}\right), \quad \text { if } g=a_{r_{g}} n k^{\prime} \\
& \leq e^{\delta \ell_{2} \epsilon} \int_{k \in K} \int_{g \in G} \psi_{\epsilon}(g) \mathcal{R}_{f \cdot \chi_{\Omega}}\left(k^{-1} g\right) d g d \nu_{o}\left(k X_{0}^{-}\right) \text {, by (7.6) } \\
& \leq e^{\delta \ell_{2} \epsilon} \int_{k \in K} \int_{g \in G} \psi_{\epsilon}(g)\left(\mathcal{R}_{f \cdot \chi_{\Omega_{\epsilon+}}}\left(k^{-1}\right)+\eta\right) d g d \nu_{o}\left(k X_{0}^{-}\right) \\
& =e^{\delta \ell_{2} \epsilon}\left(\int_{k \in K} \mathcal{R}_{f \cdot \chi_{\Omega_{\epsilon+}}}\left(k^{-1}\right) d \nu_{o}\left(k X_{0}^{-}\right)+\eta\left|\nu_{o}\right|\right) \text {, as } \int_{G} \psi_{\epsilon}(g) d g=1 \\
& =e^{\delta \ell_{2} \epsilon}\left(\int_{k \in \Omega_{\epsilon+}^{-1}} f\left(k^{-1}\right) d \nu_{o}\left(k X_{0}^{-}\right)+\eta\left|\nu_{o}\right|\right) \text {. }
\end{aligned}
$$

Since $\bigcap_{\epsilon>0} \Omega_{\epsilon+}=\bar{\Omega}$ and $\eta>0$ was arbitrarily chosen,

$$
\limsup _{\epsilon \rightarrow 0} \tilde{m}^{\mathrm{BR}}\left(f *_{\Omega} \psi_{\epsilon}\right) \leq \int_{k \in \overline{\Omega^{-1}}} f\left(k^{-1}\right) d \nu_{o}\left(k X_{0}^{-}\right) .
$$

Similarly, $\liminf \operatorname{in}_{\epsilon \rightarrow 0} \tilde{m}^{\mathrm{BR}}\left(f *_{\Omega} \psi_{\epsilon}\right) \geq \int_{k \in \operatorname{int} \Omega^{-1}} f\left(k^{-1}\right) d \nu_{o}\left(k X_{0}^{-}\right)$. Since we assume that $\nu_{o}\left(\partial\left(\Omega^{-1}\right) X_{0}^{-}\right)=0$, we obtain (7.5) .

7.2. Setup for counting results. Until the end of this section, let $V$ be a finitedimensional vector space on which $G$ acts linearly from the right and let $w_{0} \in V$. We set $H:=G_{w_{0}}$.

7.2.1. When $H$ is a symmetric subgroup of $G$. Let $H<G=\operatorname{SO}(n, 1)^{\circ}$ be a symmetric subgroup, i.e., there is a non-trivial involution $\sigma$ of $G$ such that $H^{\circ}=\left(G^{\sigma}\right)^{\circ}$ where $G^{\sigma}=\{g \in G: \sigma(g)=g\}$. There exists a Cartan involution $\theta$ of $G$ such that $\theta \circ \sigma=\sigma \circ \theta$. Let $K=G^{\theta}$. It turns out that $H^{\circ}$ is a subgroup of finite index in its normalizer $N_{G}\left(H^{\circ}\right)$, and up to a conjugation of $G, H^{\circ}=(\mathrm{SO}(k, 1) \times \mathrm{SO}(n-k))^{\circ}$ for some $0 \leq k \leq n-1$ and $K=\mathrm{SO}(n)$. Choose $o \in \mathbb{H}^{n}$ such that $G_{o}=K$. Then $\tilde{S}=H \cdot o$ is an isometric imbedding of $\mathbb{H}^{k}$ in $\mathbb{H}^{n}$. Let $\tilde{E}$ be the unit normal bundle over $\tilde{S}$.

7.2.2. When $G_{\mathbb{R} w_{0}}$ is a parabolic subgroup of $G$. Suppose that $G_{\mathbb{R} w_{0}}$ is a parabolic subgroup of $G$. Let $\theta$ be any Cartan involution of $G$ and let $K=G^{\theta}$. Then $G=G_{\mathbb{R} w_{0}} K$. Let $N$ be the unipotent radical of $G_{\mathbb{R} w_{0}}$. Let $o \in \mathbb{H}^{n}$ be such that $G_{o}=K$. Then $\tilde{S}:=N \cdot o$ is a horosphere. Let $\tilde{E} \subset \mathrm{T}^{1}\left(\mathbb{H}^{n}\right)$ be the unstable horosphere such that $\pi(\tilde{E})=\tilde{S}$ and let $H=\left(G_{\mathbb{R} w_{0}} \cap K\right) N$.

7.2.3. Common structure in both cases. Let the notation be as in any of the above $\S \$ 7.2 .1$ or 7.2.2. Let $X_{0} \in \mathrm{T}_{o}^{1}\left(\mathbb{H}^{n}\right) \cap \tilde{E}$ and let $\tilde{E}^{*}=H \cdot X_{0}$. If $H$ is symmetric and $\operatorname{codim}(\tilde{S})>1$, or if it is the parabolic case, then $\tilde{E}$ is connected and $\tilde{E}^{*}=\tilde{E}$. If $H$ is symmetric and $\operatorname{codim}(\tilde{S})=1$, then $\tilde{E}$ has two connected components, $\tilde{E}^{+}$ containing $X_{0}$ and $\tilde{E}^{-}$containing $-X_{0}$, and then either $\tilde{E}^{*}=\tilde{E}$ or $\tilde{E}^{*}=\tilde{E}^{+}$. There exists a one-parameter subgroup $A=\left\{a_{r}\right\} \subset G$ consisting of $\mathbb{R}$-diagonalizable elements such that $\mathcal{G}^{r}\left(X_{0}\right)=a_{r} X_{0}$ for all $r \in \mathbb{R}$. Let $M=G_{X_{0}}$, which coincides with $Z_{K}(A)$, i.e., the centralizer of $A$ in $K$, and $A^{ \pm}=\left\{a_{ \pm r}: r \geq 0\right\}$. Let $N$ be the expanding horospherical subgroup with respect to $\left\{a_{r}\right\}$.

When $G_{\mathbb{R} w_{0}}$ is parabolic, then $G_{w_{0}}=M N=H$ where $M=G_{\mathbb{R} w_{0}} \cap K$. Hence $N$ is the unipotent radical of $G_{\mathbb{R} w_{0}}$, so there is no conflict of notation. In the case when $H$ is symmetric, $\tilde{E}^{*}=\tilde{E}$ if and only if $G=H A^{+} K$. In all cases, we have 
$G=H A K$. Put $E=\mathbf{p}(\tilde{E}), E^{*}=\mathbf{p}\left(\tilde{E}^{*}\right)$, and in the special cases when $\tilde{E}$ is not connected, we set $E^{ \pm}=\mathbf{p}\left(\tilde{E}^{ \pm}\right)$.

7.2.4. $H A K$ decomposition of Haar measure on $G$. Note that $\tilde{E}^{*}=H X_{0} \cong H /(M \cap H)$ and recall that

$$
d \mu_{\tilde{E}}^{\mathrm{Leb}}(v)=e^{(n-1) \beta_{v^{+}}(o, \pi(v))} d m_{o}\left(v^{+}\right) .
$$

There is a Haar measure $d h$ on $H$ such that for any $\psi \in C_{c}(H)$, if we put $\bar{\psi}(h)=$ $\int_{m \in M \cap H} \psi(h m) d m$, where $d m$ denotes the probability Haar integral on $M \cap H$, then $\bar{\psi} \in C_{c}(H)^{M \cap H}=C_{c}(\tilde{E})$, and

$$
\int_{H} \psi d h=\int_{\tilde{E}} \bar{\psi} d \mu_{\tilde{E}}^{\mathrm{Leb}} .
$$

In view of the decompositions $G=H A^{+} K$ or $G=H A K$, there exists a function $\rho: \mathbb{R} \rightarrow(0, \infty)$ such that we get the following Haar measure $d g$ on $G$ : For any $\psi \in C_{c}(G)$, by [35, Theorem 8.1.1]

$$
\begin{aligned}
\int_{G} \psi d g & =\int_{k \in K} \int_{r \in R} \int_{h \in H} \rho(r) \psi\left(h a_{r} k\right) d h d r d k \text { and } \\
\rho(r) & \sim \begin{cases}e^{(n-1)|r|} & \text { if } r \rightarrow \pm \infty \text { and } H \text { is symmetric, } \\
e^{(n-1) r} & \text { if } r \rightarrow \pm \infty \text { and } G_{\mathbb{R} w_{0}} \text { is parabolic, }\end{cases}
\end{aligned}
$$

where $R=\{r \geq 0\}$ if $G=H A^{+} K$, otherwise $R=\mathbb{R}$. In fact, the Haar measure $d g$ described in Notation 7.4(1) and the Haar measure $d g$ defined in (17.8) are identical; see $₫ 8$.

7.3. Extension of Theorem $\mathbf{1 . 8}$ to $\Gamma \backslash G$ for Zariski dense $\Gamma$. The result in this subsection will enable us to state our counting theorems for general norms, provided $\Gamma$ is Zariski dense.

Let $\bar{m}^{\mathrm{BR}}$ be the measure on $\Gamma \backslash G$ which is the $M$-invariant extension of $m^{\mathrm{BR}}$. That is, for $\psi \in C_{c}(\Gamma \backslash G)$,

$$
\bar{m}^{\mathrm{BR}}(\psi):=m^{\mathrm{BR}}(\bar{\psi}),
$$

where $\bar{\psi}\left(\mathbf{p}\left(g X_{0}\right)\right)=\int_{m \in M} \psi(\Gamma g m) d m$ and $d m$ denotes the Haar probability measure on $M$.

As $M$ normalizes $N, \bar{m}^{\mathrm{BR}}$ is invariant for the right-translation action of $N$ on $\Gamma \backslash G$.

Theorem 7.6 (Flaminio-Spatzier [11, Cor. 1.6]). Suppose that $\Gamma$ is Zariski dense and $\left|m^{\mathrm{BMS}}\right|<\infty$. Then $\bar{m}^{\mathrm{BR}}$ is $N$-ergodic.

Let $H$ and $\tilde{E}$ be as in $\$ 7.2 .1$ or 7.2 .2 so that $H=G_{\tilde{E}}$. Let $d h$ be the Haar measure on $H$ defined as in (7.7). By abuse of notation, we also denote by $d h$ the measure on $\Gamma_{H} \backslash H$ induced by $d h$.

We recall that for $\Gamma$ Zariski dense, $\left|\mu_{E}^{\mathrm{PS}}\right|<\infty$ implies that the canonical map $\Gamma_{H} \backslash H \rightarrow \Gamma \backslash G$ is proper by Theorem 2.21 .

Theorem 7.7. Let $\Gamma$ be a Zariski dense discrete subgroup of $G$ such that $\left|m^{\mathrm{BMS}}\right|<$ $\infty$ and $\left|\mu_{E}^{\mathrm{PS}}\right|<\infty$. Then for any $\psi \in C_{c}(\Gamma \backslash G)$,

$$
\lim _{r \rightarrow \infty} e^{(n-1-\delta) r} \int_{h \in \Gamma_{H} \backslash H} \psi\left(\Gamma h a_{r}\right) d h=\frac{\left|\mu_{E}^{\mathrm{PS}}\right|}{\left|m^{\mathrm{BMS}}\right|} \bar{m}^{\mathrm{BR}}(\psi) .
$$


Proof. Define a measure $\lambda_{r}$ on $\Gamma \backslash G$ as follows: for any $\psi \in C_{c}(\Gamma \backslash G)$,

$$
\lambda_{r}(\psi)=e^{(n-1-\delta) r} \int_{h \in \Gamma_{H} \backslash H} \psi\left(\Gamma h a_{r}\right) d h .
$$

Let $\mathbf{q}: \Gamma \backslash G \rightarrow \mathrm{T}^{1}\left(\Gamma \backslash \mathbb{H}^{n}\right) \cong \Gamma \backslash G / M$ be the natural quotient map. Then for any $\bar{\psi} \in C_{c}\left(\mathrm{~T}^{1}\left(\Gamma \backslash \mathbb{H}^{n}\right)\right)$, we have $\bar{\psi}\left(\mathbf{q}\left(x m a_{r}\right)\right)=\bar{\psi}\left(\mathbf{q}\left(x a_{r}\right)\right)$ for any $m \in M$ and $x \in \Gamma \backslash G$, as $M$ and $A$ commute with each other, and hence

$$
\mathbf{q}_{*}\left(\lambda_{r}\right)(\psi)=\lambda_{r}(\bar{\psi} \circ \mathbf{q})=e^{(n-1-\delta) r} \int_{E} \bar{\psi}\left(v a_{r}\right) d \mu_{E}^{\mathrm{Leb}}(v) .
$$

Therefore by Theorem 1.8, $\mathbf{q}_{*}\left(\lambda_{r}\right) \rightarrow C \cdot m^{\mathrm{BR}}$, where $C=\frac{\left|\mu_{E}^{\mathrm{PS}}\right|}{\left|m^{\mathrm{BMS}}\right|}$.

In order to show that $\lambda_{r}$ weakly converges to $C \bar{m}^{\mathrm{BR}}$, it suffices to show that every sequence $\lambda_{r_{k}}$ has a subsequence converging to $C \bar{m}^{\mathrm{BR}}$.

For any sequence $r_{k} \rightarrow \infty$, since $\mathbf{q}$ is a proper map, after passing to a subsequence of $\left\{r_{k}\right\}$ there exists a measure $\lambda$ on $\Gamma \backslash G$ such that $\lambda_{r_{k}}(\phi) \rightarrow \lambda(\phi)$ for every $\phi \in$ $C_{c}(\Gamma \backslash G)$. Therefore

$$
\mathbf{q}_{*}(\lambda)=C m^{\mathrm{BR}} .
$$

For any $g \in G$, define a measure $g \lambda$ on $\Gamma \backslash G$ by $g \lambda(A)=\lambda(A g)$ for any measurable $A \subset \Gamma \backslash G$. Now for any $\psi \in C_{c}(\Gamma \backslash G)$,

$$
\begin{aligned}
\int_{m \in M}(m \lambda)(\psi) d m & =\int_{m \in M} \int_{\Gamma \backslash G} \psi(x m) d \lambda(x) d m \\
& =\overline{\mathbf{q}}_{*}(\lambda)(\bar{\psi})=C m^{\mathrm{BR}}(\bar{\psi})=C \bar{m}^{\mathrm{BR}}(\psi) .
\end{aligned}
$$

Claim 1. $\lambda$ is $N$-invariant.

Proof of Claim 1. Due to Lemma 2.1, the map $h \mapsto h X_{0}^{+}$is a submersion, and hence there exists a neighborhood $\Omega$ of $e$ in $N$ and a continuous injective map $\sigma: \Omega \rightarrow H$ such that $\sigma(e)=e$ and $\sigma(z) X_{0}^{+}=z X_{0}^{+}$for all $z \in \Omega$.

Fix $z \in \Omega$, let $z_{k}:=a_{r_{k}} z a_{-r_{k}}$, and let $h_{k}=\sigma\left(z_{k}\right)$ for all large $k$. Then $b_{k}=$ $z_{k}^{-1} h_{k} \in G_{X_{0}^{+}}=M A N^{-}$. Therefore $b_{k} \rightarrow e$ and $a_{-r_{k}} b_{k} a_{r_{k}} \rightarrow e$ as $k \rightarrow \infty$.

Let $\psi \in C_{c}(\Gamma \backslash G)$. Given $\epsilon>0$ and $x \in \Gamma \backslash G$, set

$$
\psi_{\epsilon+}(x)=\sup _{g \in U_{\epsilon}} \psi(x g) \quad \text { and } \quad \psi_{\epsilon-}=\inf _{g \in U_{\epsilon}} \psi(x g) .
$$

Since $\psi$ is uniformly continuous and $a_{r_{k}} z=h_{k} b_{k}^{-1} a_{r_{k}}=h_{k} a_{r_{k}}\left(a_{-r_{k}} b_{k}^{-1} a_{r_{k}}\right)$, we have for all large $k$ and for all $x \in \Gamma \backslash G$,

$$
\psi_{\epsilon-}\left(x h h_{k} a_{r_{k}}\right) \leq \psi\left(x a_{r_{k}} z\right) \leq \psi_{\epsilon+}\left(x h_{k} a_{r_{k}}\right) .
$$

Since the measure $d h$ is $H$-invariant,

$$
\int_{h \in \Gamma_{H} \backslash H} \psi\left(\Gamma h a_{r_{k}} z\right) d h \leq \int_{\Gamma_{H} \backslash H} \psi_{\epsilon+}\left(\Gamma h h_{k} a_{r_{k}}\right) d h=\int_{\Gamma_{H} \backslash H} \psi_{\epsilon+}\left(\Gamma h a_{r_{k}}\right) d h .
$$

Similarly, we get a lower bound in terms of $\psi_{\epsilon-}$. Since $\lambda_{r_{k}} \rightarrow \lambda$ as $k \rightarrow \infty$,

$$
\lambda\left(\psi_{\epsilon-}\right) \leq \int_{\Gamma \backslash G} \psi(x z) d \lambda(x) \leq \lambda\left(\psi_{\epsilon+}\right) .
$$

Since $\psi \in C_{c}(\Gamma \backslash G)$, we have that $\lambda\left(\psi_{\epsilon \pm}\right) \rightarrow \lambda(\psi)$ as $\epsilon \rightarrow 0$. Therefore the $z$-action preserves $\lambda$. This proves Claim 1 . 
Claim 2. $\lambda=C \bar{m}^{\mathrm{BR}}$.

Proof of Claim 2. By (7.10), it is enough to show that $\lambda$ is $M$-invariant. For any $\epsilon>0$, define a measure $\eta_{\epsilon}$ on $\Gamma \backslash G$ by

$$
\eta_{\epsilon}:=\frac{1}{\left|M_{\epsilon}\right|} \int_{m \in M_{\epsilon}} m \lambda d m
$$

where $\left|M_{\epsilon}\right|=\int_{M_{\epsilon}} d m$. Then since $M$ normalizes $N, \eta_{\epsilon}$ is $N$-invariant. By (7.10)

$$
\eta_{\epsilon} \ll \bar{m}^{\mathrm{BR}} \text {. }
$$

Therefore, since $\bar{m}^{\mathrm{BR}}$ is $N$-ergodic by Theorem 7.6. there exists $c_{\epsilon}>0$ such that $\eta_{\epsilon}=c_{\epsilon} \bar{m}^{\mathrm{BR}}$. Thus $\eta_{\epsilon}$ is $M$-invariant, as $\bar{m}^{\mathrm{BR}}$ is $M$-invariant.

If $\lambda$ is not $M$-invariant, there exist $\psi \in C_{c}(\Gamma \backslash G), m_{0} \in M$ and $\beta>0$ such that $\lambda\left(m_{0} \cdot \psi\right) \geq \lambda(\psi)+\beta$. There exists $\epsilon>0$ such that for all $m \in M_{\epsilon}, \lambda\left(\left(m m_{0}\right) \psi\right) \geq$ $\lambda(m \cdot \psi)+\beta / 2$. This implies that $\eta_{\epsilon}\left(m_{0} \psi\right) \geq \eta_{\epsilon}(\psi)+\beta / 2$, which is a contradiction to the $M$-invariance of $\eta_{\epsilon}$. Hence Claim 2 is proved.

As noted before, this completes the proof of Theorem 7.7 .

7.4. Statements of counting theorems. Now we describe the main counting results of this section. In the next two theorems, Theorems 17.8, and 7.10, we suppose that the following conditions hold for $w_{0} \in V$ and $\Gamma$ a non-elementary discrete torsion-free subgroup of $G$ :

(1) $w_{0} \Gamma$ is discrete.

(2) $H$ is a symmetric subgroup of $G$, or $G_{\mathbb{R} w_{0}}$ is a parabolic subgroup of $G$.

(3) $\left|m^{\mathrm{BMS}}\right|<\infty$ and $\left|\mu_{E}^{\mathrm{PS}}\right|<\infty$.

Let $\lambda \in \mathbb{N}$ be the log of the largest eigenvalue of $a_{1}$ on $\mathbb{R}-\operatorname{span}\left(w_{0} G\right)$, and set

$$
w_{0}^{\lambda}:=\lim _{r \rightarrow \infty} \frac{w_{0} a_{r}}{e^{\lambda r}} \text { and } w_{0}^{-\lambda}:=\lim _{r \rightarrow \infty} \frac{w_{0} a_{-r}}{e^{\lambda r}} .
$$

Theorem 7.8 (Counting in sectors). Let $\|\cdot\|$ be a norm on $V$ satisfying

$$
\left\|w_{0}^{ \pm \lambda} m k\right\|=\left\|w_{0}^{ \pm \lambda} k\right\|, \text { for all } m \in M \text { and } k \in K,
$$

and set $B_{T}:=\{v \in V:\|v\|<T\}$.

(1) For any Borel measurable $\Omega \subset K$ such that $M \Omega=\Omega$ and $\nu_{o}\left(\partial\left(\Omega^{-1} X_{0}^{-}\right)\right)=$ 0 ,

$$
\lim _{T \rightarrow \infty} \frac{\#\left(w_{0} \Gamma \cap B_{T} \cap\left(w_{0} A^{+} \Omega\right)\right)}{T^{\delta / \lambda}}=\frac{\mu_{E}^{\mathrm{PS}}\left(E^{*}\right)}{\delta \cdot\left|m^{\mathrm{BMS}}\right|} \int_{k \in \Omega^{-1}}\left\|w_{0}^{\lambda} k^{-1}\right\|^{-\delta / \lambda} d \nu_{o}\left(k X_{0}^{-}\right) .
$$

(2) For the full count in a ball, we get

$$
\begin{aligned}
& \lim _{T \rightarrow \infty} \frac{\#\left(w_{0} \Gamma \cap B_{T}\right)}{T^{\delta / \lambda}} \\
& =\left\{\begin{array}{l}
\frac{\mu_{E}^{\mathrm{PS}}(E)}{\delta \cdot\left|m^{\mathrm{BMS}}\right|} \int_{k \in K}\left\|w_{0}^{\lambda} k^{-1}\right\|^{-\delta / \lambda} d \nu_{o}\left(k X_{0}^{-}\right)>0, \text { if } \tilde{E}=G_{w_{0}} \cdot X_{0}, \\
\sum_{ \pm} \frac{\mu_{E}^{\mathrm{PS}}\left(E^{ \pm}\right)}{\delta \cdot\left|m^{\mathrm{BMS}}\right|} \int_{k \in K}\left\|w_{0}^{ \pm \lambda} k^{-1}\right\|^{-\delta / \lambda} d \nu_{o}\left(k X_{0}^{\mp}\right)>0, \text { otherwise. }
\end{array}\right.
\end{aligned}
$$

Remark 7.9. (1) By [13, Lemma 4.2], we have $w_{0}^{\lambda} \neq 0$. Also, if $H$ is symmetric, then $w_{0}^{-\lambda} \neq 0$. 
(2) Since $w_{0} \Gamma$ is discrete, $H \Gamma$ is closed in $G$, and hence $\Gamma H$ is closed in $G$. It follows that the canonical imbedding $(\Gamma \cap H) \backslash H \rightarrow \Gamma \backslash G$ is a proper injective map. This properness follows from a suitable open mapping theorem in the category of locally compact Hausdorff second countable topological group actions. Therefore the map $\left(\Gamma \cap G_{\tilde{S}}\right) \backslash \tilde{S} \rightarrow \Gamma \backslash \mathbb{H}^{n}$ is a proper map. In particular, $E$ and $E^{ \pm}$are closed subsets of $\mathrm{T}^{1}\left(\Gamma \backslash \mathbb{H}^{n}\right)$.

(3) The condition (17.12) holds if $\|\cdot\|$ is $K$-invariant as inTheorem 1.2 There exists a Weyl group element $k_{0} \in K$ such that $k_{0}^{-1} a_{r} k_{0}=a_{-r}$ for all $r \in \mathbb{R}$. Then $w_{0}^{-\lambda}=w_{0}^{\lambda} k_{0}$. Therefore if $\|\cdot\|$ is $K$-invariant, then $\left\|w_{0}^{ \pm \lambda} k\right\|=\left\|w_{0}^{\lambda}\right\|$ for all $k \in K$. Then the limit (7.13) becomes (1.1). Thus Theorem 7.8 implies Theorem 1.2 .

(4) When $\Gamma$ is Zariski dense in $G$, Theorem 7.8 holds for any norm on $V$ without the condition (7.12) and for the $\Omega$ without the $M$-invariance condition. See $\$ 7.7$ for details.

(5) Since $w_{0}^{ \pm \lambda}$ is fixed by $H \cap Z_{K}(A)$, if $M=Z_{K}(A) \subset H$, then condition (7.12) holds for any norm on $V$. We have $M \subset H$ in the parabolic case. In the case when $H$ is symmetric, if $\tilde{S}$ is a single point or $\tilde{S}$ is of codimension one, then $M \subset H$.

Theorem 7.10 (Counting in cones). Suppose further that $\Gamma$ is Zariski dense in $G$. Let $\Theta$ be a measurable subset of $V$ and let

$$
\Omega_{ \pm}=\left\{k \in K: w_{0}^{ \pm \lambda} k \in \mathbb{R}^{+} \Theta\right\} .
$$

If $\nu_{o}\left(\partial\left(\Omega_{ \pm}^{-1} X_{0}^{\mp}\right)\right)=0$, then for any norm $\|\cdot\|$ on $V$,

$$
\begin{aligned}
& \lim _{T \rightarrow \infty} \frac{\#\left(w_{0} \Gamma \cap B_{T} \cap \mathbb{R}^{+} \Theta\right)}{T^{\delta / \lambda}}=\frac{1}{\delta \cdot\left|m^{\mathrm{BMS}}\right|} \\
& \times\left\{\begin{array}{l}
\mu_{E}^{\mathrm{PS}}(E) \int_{k \in \Omega_{+}^{-1}}\left\|w_{0}^{\lambda} k^{-1}\right\|^{-\delta / \lambda} d \nu_{o}\left(k X_{0}^{-}\right), \text {if } \tilde{E}=H X_{0}, \\
\sum \mu_{E}^{\mathrm{PS}}\left(E^{ \pm}\right) \int_{k \in \Omega_{ \pm}^{-1}}\left\|w_{0}^{ \pm \lambda} k^{-1}\right\|^{-\delta / \lambda} d \bar{\nu}_{o}\left(k X_{0}^{\mp}\right) \text {, otherwise. }
\end{array}\right.
\end{aligned}
$$

Note that if $\Gamma$ is Zariski dense in $G$ and if $\partial\left(\Omega_{ \pm}\right)$is contained in a countable union of proper real algebraic subvarieties of $\partial \mathbb{H}^{n}$, then $\nu_{o}\left(\partial\left(\Omega_{ \pm}\right)\right)=0$ (see [11, Corollary 1.4] and [23, Remark 1.7(2)]).

7.5. Proof of the counting statements. We follow the counting technique of [9] and [10]. For a Borel subset $\Omega \subset K$ satisfying the condition of Theorem 7.8, we set

$$
B_{T}(\Omega)=B_{T} \cap w_{0} A^{+} \Omega
$$

and define the following counting function on $\Gamma \backslash G$ :

$$
F_{B_{T}(\Omega)}(g):=\sum_{\gamma \in \Gamma_{w_{0}} \backslash \Gamma} \chi_{B_{T}(\Omega)}\left(w_{0} \gamma g\right) .
$$

We note that

$$
F_{B_{T}(\Omega)}(e)=\#\left(w_{0} \Gamma \cap B_{T}(\Omega)\right)=\#\left(w_{0} \Gamma \cap B_{T} \cap\left(w_{0} A^{+} \Omega\right)\right) .
$$

For $\psi_{1}, \psi_{2} \in C_{c}(\Gamma \backslash G)$, we set $\left\langle\psi_{1}, \psi_{2}\right\rangle:=\int_{\Gamma \backslash G} \psi_{1}(g) \psi_{2}(g) d g$.

Let $\psi \in C_{c}(\Gamma \backslash G)$. Then by (7.8),

$$
\begin{aligned}
& \left\langle F_{B_{T}(\Omega)}, \psi\right\rangle=\int_{\Gamma_{w_{0} \backslash G}} \chi_{B_{T}(\Omega)}\left(w_{0} g\right) \psi(g) d g \\
& =\int_{k \in \Omega} \int_{\left\{r \geq 0:\left\|w_{0} a_{r} k\right\|<T\right\}}\left(\int_{[h] \in \Gamma_{w_{0} \backslash H}} \psi\left(h a_{r} k\right) d h\right) \rho(r) d r d k .
\end{aligned}
$$


For any $k \in K$ and $T>0$, define

$$
r(k, T)=\sup \left\{r>0:\left\|w_{0} a_{r} k\right\|<T\right\} .
$$

Let $\lambda_{1}$ be the log of the largest eigenvalue of $a_{1}$ on $V$ strictly less than $e^{\lambda}$. Then by (7.11) there exist $C_{1} \geq 1$ and $r_{1} \geq 0$ such that

$$
\left\|w_{0} a_{r} k-e^{\lambda r} w_{0}^{\lambda} k\right\| \leq C_{1} e^{\lambda_{1} r}, \text { for all } k \in K \text { and } r \geq r_{1} .
$$

Put $\epsilon_{0}=\left(\lambda-\lambda_{1}\right) / \lambda>0$ and $C_{2}=2 C_{1} / \inf _{k \in K}\left\|w_{0}^{\lambda} k\right\|$. Let $T_{1} \geq 1$ be such that $C_{2} T_{1}^{-\epsilon_{0}} \leq 1 / 2$ and $(1 / 2)\left(T_{1} / \sup _{k \in K}\left\|w_{0}^{\lambda} k\right\|\right)^{1 / \lambda} \geq e^{r_{1}}$. For $T \geq T_{1}$, we define functions $r_{ \pm}(k, T)$ via

$$
e^{r_{ \pm}(k, T)}=\left(T /\left\|w_{0}^{\lambda} k\right\|\right)^{1 / \lambda}\left(1 \pm C_{2} T^{-\epsilon_{0}}\right) .
$$

Then by elementary calculation using (7.18),

$$
r_{-}(k, T) \leq r(k, T) \leq r_{+}(k, T) \text {, for all } T \geq T_{1} \text { and } k \in K .
$$

By (7.12),

$$
r_{ \pm}(m k, T)=r_{ \pm}(k, T), \text { for all } m \in M \text { and } k \in K .
$$

We note that by (7.19), given $\epsilon>0$, for $T_{1}(\epsilon)$ sufficiently large,

$$
e^{\delta r_{ \pm}(k, T)}=(1+O(\epsilon))\left(T /\left\|w_{0}^{\lambda} k\right\|\right)^{\delta / \lambda} \text { for all } T \geq T_{1}(\epsilon) .
$$

Proposition 7.11. For any non-negative $\psi \in C_{c}(\Gamma \backslash G)$,

$$
\begin{aligned}
& \int_{k \in \Omega} \int_{0}^{r_{-}(k, T)} \rho(r)\left(\int_{E^{*}} \psi_{k}\left(\mathcal{G}^{r}(v)\right) d \mu_{E}^{\mathrm{Leb}}(v)\right) d r d k \leq\left\langle F_{B_{T}(\Omega)}, \psi\right\rangle \\
& \leq \int_{k \in \Omega} \int_{0}^{r_{+}{ }^{(k, T)}} \rho(r)\left(\int_{E^{*}} \psi_{k}\left(\mathcal{G}^{r}(v)\right) d \mu_{E}^{\mathrm{Leb}}(v)\right) d r d k,
\end{aligned}
$$

where $\psi_{k} \in C_{c}(\Gamma \backslash G)^{M} \cong C_{c}\left(\mathrm{~T}^{1}\left(\Gamma \backslash \mathbb{H}^{n}\right)\right)$ is given by

$$
\psi_{k}(g)=\int_{m \in M} \psi(g m k) d m .
$$

Proof. By (7.7), (7.8), (7.16), (7.20), (7.21) and Lemma 7.1, we get

$$
\begin{aligned}
& \left\langle F_{B_{T}(\Omega)}, \psi\right\rangle \\
& =\int_{k \in \Omega} \int_{\left\{r \geq 0:\left\|w_{0} a_{r} k\right\|<T\right\}}\left(\int_{[h] \in \Gamma_{w_{0}} \backslash H} \psi\left(h a_{r} k\right) d h\right) \rho(r) d r d k \\
& \leq \int_{k \in \Omega} \int_{0}^{r+(k, T)}\left(\int_{[h] \in \Gamma_{w_{0}} \backslash H} \psi\left(h a_{r} k\right) d h\right) \rho(r) d r d k \\
& =\int_{k \in \Omega} \int_{0}^{r_{+}(k, T)}\left(\int_{[h] \in \Gamma_{w_{0}} \backslash H} \int_{m \in M} \psi\left(h a_{r} m k\right) d m d h\right) \rho(r) d k, \text { as } M \Omega=\Omega \\
& =\int_{k \in \Omega} \int_{0}^{r_{+}(k, T)}\left(\int_{[h] \in \Gamma_{w_{0}} \backslash H} \psi_{k}\left(h a_{r}\right) d h\right) \rho(r) d r d k \\
& =\int_{k \in \Omega} \int_{0}^{r_{+}(k, T)} \rho(r)\left(\int_{E^{*}} \psi_{k}\left(\mathcal{G}^{r}(v)\right) d \mu_{E}^{\mathrm{Leb}}(v)\right) d r d k .
\end{aligned}
$$

The other inequality is proved similarly.

Proposition 7.12. For any $\psi \in C_{c}(\Gamma \backslash G)$, we have

$$
\lim _{T \rightarrow \infty} T^{-\delta / \lambda}\left\langle F_{B_{T}(\Omega)}, \psi\right\rangle=\frac{\mu_{E}^{\mathrm{PS}}\left(E^{*}\right)}{\delta \cdot\left|m^{\mathrm{BMS}}\right|} \cdot m^{\mathrm{BR}}\left(\xi_{w_{0}} * \Omega \psi\right),
$$

where $\xi_{w_{0}}(k)=\left\|w_{0}^{\lambda} k\right\|^{-\delta / \lambda}$. 
Proof. Without loss of generality, we may assume that $\psi$ is non-negative. For any $\epsilon>0$ and $k \in K$, by Theorem [1.8 and (7.9), there exists $r_{0}>0$ such that for any $r>r_{0}$,

$$
\begin{gathered}
e^{(n-1-\delta) r} \int_{v \in E^{*}} \psi_{k}\left(\mathcal{G}^{r}(v)\right) d \mu_{E}^{\mathrm{Leb}}(v)=\frac{\mu_{E}^{\mathrm{PS}}\left(E^{*}\right) \cdot m^{\mathrm{BR}}\left(\psi_{k}\right)}{\left|m^{\mathrm{BMS}}\right|}+O(\epsilon), \\
\rho(r)=(1+O(\epsilon)) e^{(n-1) r} .
\end{gathered}
$$

Since $\psi \in C_{c}(\Gamma \backslash G)$, the map $K \ni k \mapsto \psi_{k}$ is continuous with respect to the sup-norm on $C_{c}\left(\mathrm{~T}^{1}\left(\mathbb{H}^{n}\right)\right)$. Therefore, since $K$ is compact, we can choose $r_{0}>0$ independent of $k \in K$. Now for sufficiently large $T>1$,

$$
\begin{aligned}
& \int_{r_{0}}^{r^{ \pm}(k, T)} \rho(r) \int_{E^{*}} \psi_{k}\left(\mathcal{G}^{r}(v)\right) d \mu_{E}^{\mathrm{Leb}}(v) d r \\
& =\int_{r_{0}}^{r_{ \pm}(k, T)} \rho(r) e^{(-n+1+\delta) r}\left(e^{(n-1-\delta) r} \int_{E^{*}} \psi_{k}\left(\mathcal{G}^{r}(v)\right) d \mu_{E}^{\mathrm{Leb}}(v)\right) d r \\
& =\left(\frac{\mu_{E}^{\mathrm{PS}}\left(E^{*}\right) \cdot m^{\mathrm{BR}}\left(\psi_{k}\right)}{\left|m^{\mathrm{BMS}}\right|}+O(\epsilon)\right)(1+O(\epsilon)) \int_{r_{0}}^{r_{ \pm}(k, T)} e^{\delta r} d r \\
& =\frac{\mu_{E}^{\mathrm{PS}}\left(E^{*}\right) \cdot m^{\mathrm{BR}}\left(\psi_{k}\right)}{\left|m^{\mathrm{BMS}}\right|} \cdot \frac{T^{\delta / \lambda}\left\|w_{0}^{\lambda} k\right\|^{-\delta / \lambda}}{\delta}+O(\epsilon) T^{\delta / \lambda}+O\left(e^{\delta r_{0}}\right),
\end{aligned}
$$

where the last equation follows from (7.22) for sufficiently large $T$.

Since $E \subset \mathrm{T}^{1}\left(\Gamma \backslash \mathbb{H}^{n}\right)$ is a closed subset, $\psi \in C_{c}(\Gamma \backslash G)$ and $K$ is compact, it follows that for fixed $r_{0}>1$, we have

$$
\sup _{|r| \leq r_{0}, k \in K} \int_{E} \psi_{k}\left(v a_{r}\right) d \mu_{E}^{\mathrm{Leb}}(v)=O(1) .
$$

Hence

$$
\int_{\left\{r:\left\|w_{0} a_{r} k\right\|<T,|r| \leq r_{0}\right\}} \rho(r) \int_{E} \psi_{k}\left(\mathcal{G}^{r}(v)\right) d \mu_{E}^{\mathrm{Leb}}(v) d r=O\left(e^{(n-1) r_{0}}\right) .
$$

By Proposition 7.11, (7.25) and (7.26),

$$
\lim _{T \rightarrow \infty} \frac{\left\langle F_{B_{T}(\Omega)}, \psi\right\rangle}{T^{\delta / \lambda}}=\frac{\mu_{E}^{\mathrm{PS}}\left(E^{*}\right)}{\delta \cdot\left|m^{\mathrm{BMS}}\right|} \cdot \int_{k \in \Omega}\left\|w_{0}^{\lambda} k\right\|^{-\delta / \lambda} m^{\mathrm{BR}}\left(\psi_{k}\right) d k+O(\epsilon) .
$$

Since $\epsilon>0$ is arbitrary, we finish the proof.

Lemma 7.13 (Strong wavefront lemma). There exist $\ell>1$ and $\epsilon_{0}>0$ such that for any $0<\epsilon<\epsilon_{0}$ and $g=h a k \in H A^{+} K$ with $\|a\| \geq 2$,

$$
g U_{\epsilon} \subset h\left(H \cap U_{\ell \epsilon}\right) a\left(A \cap U_{\ell \epsilon}\right) k\left(K \cap U_{\ell \epsilon}\right),
$$

where $\|g\|$ denotes the distance of $g$ from e in $G$ which is K-invariant.

Proof. If $H$ is symmetric, the result follows from [14, Theorem 4.1].

Now suppose that $H=N$ is horospherical. We may assume that the distance from $e$ in $G$ is invariant under conjugation by elements of $K$. Let $u \in U_{\epsilon}$. Then $k u k^{-1} \in U_{\epsilon}$. Write $k u k^{-1}=h_{1} a_{1} k_{1}$, where $h_{1} \in H \cap U_{\ell \epsilon}, a_{1} \in A \cap U_{\ell \epsilon}$ and $k_{1} \in K \cap U_{\ell \epsilon}$ for some $\ell \geq 1$ independent of $\epsilon$. Now

$$
g u=h a k u=h a\left(k u k^{-1}\right) k=\left(h\left(a h_{1} a^{-1}\right)\right)\left(a a_{1}\right) k\left(k^{-1} k_{1} k\right) .
$$

Since $a \in A^{+}$and $h_{1} \in H=N$, by (7.1), $\left\|a h_{1} a^{-1}\right\| \leq\left\|h_{1}\right\|$. Also, $\left\|k^{-1} k_{1} k\right\|=\left\|k_{1}\right\|$. Hence $g u$ has the required form. 
Proof of Theorem 7.8(1). By the assumption that $\nu_{o}\left(\partial\left(\Omega^{-1}\right)\right)=0$, for all sufficiently small $\epsilon>0$, there exists an $\epsilon$-neighborhood $K_{\epsilon}$ of $e$ in $K$ such that for $\Omega_{\epsilon+}=\Omega K_{\epsilon}$ and $\Omega_{\epsilon-}=\bigcap_{k \in K_{\epsilon}} \Omega k$,

$$
\lim _{\epsilon \rightarrow 0} \nu_{o}\left(\Omega_{\epsilon+}^{-1}-\Omega_{\epsilon-}^{-1}\right)=0 .
$$

Let $\ell>1$ as in Lemma 7.13, Then for $T \gg 1$,

$$
B_{T}(\Omega) U_{\ell^{-1} \epsilon} \subset B_{(1+\epsilon) T}\left(\Omega_{\epsilon+}\right) \quad \text { and } \quad B_{(1-\epsilon) T}\left(\Omega_{\epsilon-}\right) \subset \bigcap_{u \in U_{\ell^{-1} \epsilon}} B_{T}(\Omega) u .
$$

Let $\psi_{\epsilon} \in C_{c}(G)$ be a non-negative function supported on $U_{\ell^{-1} \epsilon}$ and $\int \psi_{\epsilon} d g=1$, and let $\Psi_{\epsilon} \in C_{c}(\Gamma \backslash G)$ be the $\Gamma$-average of $\psi_{\epsilon}$ :

$$
\Psi_{\epsilon}(g):=\sum_{\gamma \in \Gamma} \psi_{\epsilon}(\gamma g)
$$

Then $F_{B_{(1-\epsilon) T}\left(\Omega_{\epsilon-}\right)}(g) \leq F_{B_{T}(\Omega)}(e) \leq F_{B_{(1+\epsilon) T}\left(\Omega_{\epsilon+}\right)}(g)$ for all $g \in U_{\ell^{-1} \epsilon}$. Therefore, by integrating against $\Psi_{\epsilon}$, we have

$$
\left\langle F_{B_{(1-\epsilon) T}\left(\Omega_{\epsilon-}\right)}, \Psi_{\epsilon}\right\rangle \leq F_{B_{T}(\Omega)}(e) \leq\left\langle F_{B_{(1+\epsilon) T}\left(\Omega_{\epsilon+}\right)}, \Psi_{\epsilon}\right\rangle .
$$

Let $\xi_{w_{0}}$ be as defined in Proposition 7.12. By Proposition 7.5, for any $\eta>0$, there exists $\epsilon>0$ such that

$$
m^{\mathrm{BR}}\left(\xi_{w_{0}} *_{\Omega} \Psi_{\epsilon}\right)=\tilde{m}^{\mathrm{BR}}\left(\xi_{w_{0}} *_{\Omega} \psi_{\epsilon}\right)=\int_{k \in \Omega^{-1}} \xi_{w_{0}}\left(k^{-1}\right) d \nu_{o}\left(k X_{0}^{-}\right)+O(\eta) .
$$

Therefore by Proposition 7.12 ,

$$
\begin{aligned}
& \lim _{T \rightarrow \infty} T^{-\delta / \lambda} \cdot\left\langle F_{B_{(1 \pm \epsilon) T}\left(\Omega_{\epsilon \pm}\right)}, \Psi_{\epsilon}\right\rangle \\
& =\frac{\mu_{E}^{\mathrm{PS}}\left(E^{*}\right)}{\delta \cdot\left|m^{\mathrm{BMS}}\right|} \cdot \int_{k \in \Omega_{\epsilon \pm}^{-1}} \xi_{w_{0}}\left(k^{-1}\right) d \nu_{o}\left(k X_{0}^{-}\right)+O(\eta) .
\end{aligned}
$$

In view of (7.27), we get

$$
\lim _{T \rightarrow \infty} \frac{F_{B_{T}(\Omega)}(e)}{T^{\delta / \lambda}}=\frac{\mu_{E}^{\mathrm{PS}}\left(E^{*}\right)}{\delta \cdot\left|m^{\mathrm{BMS}}\right|} \cdot \int_{k \in \Omega^{-1}} \xi_{w_{0}}\left(k^{-1}\right) d \nu_{o}\left(k X_{0}^{-}\right)+O(\eta) .
$$

Since $\eta>0$ is arbitrarily chosen, we finish the proof of (1).

Proposition 7.14. Suppose that $H=G_{w_{0}}$ is symmetric and that $G \neq H A^{+} K$. Let $\Omega \subset M \backslash K$ such that $\nu_{o}\left(\partial\left(\Omega^{-1} X_{0}^{+}\right)\right)=0$. Then

$$
\begin{gathered}
\lim _{T \rightarrow \infty} \frac{\#\left(w_{0} \Gamma \cap B_{T} \cap w_{0} A^{-} \Omega\right)}{T^{\delta / \lambda}} \\
=\frac{\mu_{E}^{\mathrm{PS}}\left(E^{-}\right)}{\delta \cdot\left|m^{\mathrm{BMS}}\right|} \int_{k \in \Omega^{-1}}\left\|w_{0}^{-\lambda} k^{-1}\right\|^{-\delta / \lambda} d \nu_{o}\left(k X_{0}^{+}\right) .
\end{gathered}
$$

Proof. For $k \in K$ and $T>0$, let $s(k, T)=\sup \left\{r>0:\left\|w_{0} a_{-r} k\right\|<T\right\}$. Then there exist $A_{0}>0$ and $T_{0}>0$ such that if we define $s_{ \pm}(k, T)$ via

$$
e^{s_{ \pm}(k, T)}=\left(1 \pm A_{0} T^{-\epsilon_{0}}\right)\left(T /\left\|w_{0}^{-\lambda} k\right\|\right)^{1 / \lambda},
$$

then for all $T \geq T_{0}$, we have $s_{-}(k, T) \leq s(k, T) \leq s_{+}(k, T)$. 
By Theorem 1.8, for any $\phi \in C_{c}\left(\Gamma \backslash \mathrm{T}^{1}\left(\mathbb{H}^{n}\right)\right)$, we have

$$
\begin{aligned}
& \lim _{r \rightarrow \infty} e^{(n-1-\delta) r} \int_{E^{+}} \phi\left(\mathcal{G}^{-r}(v)\right) d \mu_{E}^{\mathrm{Leb}}(v) \\
& =\lim _{r \rightarrow \infty} e^{(n-1-\delta) r} \int_{E^{-}} \tilde{\phi}\left(\mathcal{G}^{r}(v)\right) d \mu_{E}^{\mathrm{Leb}}(v)=\frac{\mu_{E}^{\mathrm{PS}}\left(E^{-}\right)}{\delta \cdot\left|m^{\mathrm{BMS}}\right|} \cdot m^{\mathrm{BR}}(\tilde{\phi}),
\end{aligned}
$$

where $\tilde{\phi}(v)=\phi(-v)$.

Let $B_{T}^{-}(\Omega)=B_{T} \cap w_{0} A^{-} \Omega$ and

$$
F_{B_{T}^{-}(\Omega)}(g):=\sum_{\gamma \in \Gamma_{w_{0}} \backslash \Gamma} \chi_{B_{T}^{-}(\Omega)}\left(w_{0} \gamma g\right) .
$$

In view of these observations, by arguing as in the proof of Proposition 7.12 we get that for any $\psi \in C_{c}(\Gamma \backslash G)$,

$$
\begin{aligned}
& \lim _{T \rightarrow \infty} T^{-\delta / \lambda}\left\langle F_{B_{T}^{-}(\Omega)}, \psi\right\rangle \\
& =\lim _{T \rightarrow \infty} T^{-\delta / \lambda} \int_{k \in \Omega} \int_{\left\{r>0:\left\|w_{0} a_{-r} k\right\|<T\right\}}\left[\int_{[h] \in \Gamma_{w_{0}} \backslash H} \psi\left(h a_{-r} k\right) d h\right] \rho(r) d r d k \\
& =\frac{\mu_{E}^{\mathrm{PS}}\left(E^{-}\right)}{\delta \cdot\left|m^{\mathrm{BMS}}\right|} \cdot \int_{k \in \Omega}\left\|w_{0}^{-\lambda} k\right\|^{-\delta / \lambda} m^{\mathrm{BR}}\left(\psi_{k_{0} k}\right) d k,
\end{aligned}
$$

where $k_{0} \in K$ represents an element of the Weyl group such that $k_{0} a_{r} k_{0}^{-1}=$ $a_{-r}$ and $k_{0} X_{0}^{-}=X_{0}^{+}$. Now (7.30) follows from the arguments as in the proof of Theorem $7.8(1)$.

Remark 7.15. If $G_{\mathbb{R} w_{0}}$ is parabolic, then $w_{0} a_{r} \rightarrow 0$ as $r \rightarrow-\infty$. Since $w_{0} \Gamma$ is discrete,

$$
\#\left(w_{0} \Gamma \cap w_{0} A^{-} K\right)<\infty .
$$

Proof of Theorem 7.8 (2). If $G=H A^{+} K$, then (2) follows from (1) by putting $\Omega=K$.

If $H$ is symmetric and $G \neq H A^{+} K$, then $G=H A^{+} K \sqcup H A^{-} K$, and then (2) follows by combining (1) and Proposition 7.14 and putting $\Omega=K$.

If $G_{\mathbb{R} w_{0}}$ is parabolic, (7.13) follows from Theorem 7.8 and (7.31).

7.6. Counting in bisectors of $H A^{+} K$ coordinates. We state a counting result for bisectors in $H A^{+} K$ coordinates. For any $g \in H A^{+} K$, we set $a(g)$ to be the $A^{+}$-component of $g$, which is unique. Consider bounded Borel subsets $\Omega_{1} \subset H$ and $\Omega_{2} \subset K$ with $\Omega_{1}(H \cap M)=\Omega_{1}$ and $M \Omega_{2}=\Omega_{2}$. Set

$$
N_{T}\left(\Omega_{1}, \Omega_{2}\right)=\#\left(\Gamma \cap \Omega_{1} A_{T}^{+} \Omega_{2}\right),
$$

where $A_{T}^{+}=\left\{a_{r} \in A^{+}: e^{r}<T\right\}$. For the sake of simplicity, we assume that the projection map $\Omega_{1} \rightarrow \Gamma \backslash G$ is injective.

Theorem 7.16. If $\mu_{E}^{\mathrm{PS}}\left(\partial\left(\Omega_{1}\left(X_{0}\right)\right)\right)=\nu_{o}\left(\partial\left(\Omega_{2}^{-1}\left(X_{0}^{-}\right)\right)\right)=0$, then

$$
\lim _{T \rightarrow \infty} \frac{N_{T}\left(\Omega_{1}, \Omega_{2}\right)}{T^{\delta}}=\frac{1}{\delta \cdot\left|m^{\mathrm{BMS}}\right|} \mu_{E}^{\mathrm{PS}}\left(\Omega_{1}\left(X_{0}\right)\right) \cdot \nu_{o}\left(\Omega_{2}^{-1}\left(X_{0}^{-}\right)\right) .
$$

This result for $H=K$ was also obtained by Roblin 31] by a different approach. When $\Gamma$ is a lattice in a semisimple Lie group $G$ and $H=K$, the analogue of Theorem 7.16] was obtained in [12]. 
Proof. We define the following function on $\Gamma \backslash G$ :

$$
F_{T, \Omega_{1}, \Omega_{2}}(g):=\sum_{\gamma \in \Gamma} \chi_{\Omega_{1} A_{T}^{+} \Omega_{2}}(\gamma g) .
$$

For $\psi \in C_{c}(\Gamma \backslash G)$, given $\epsilon>0$, by Theorem 3.6 for sufficiently large $T>1$,

$$
\begin{aligned}
& \left\langle F_{T, \Omega_{1}, \Omega_{2}}, \psi\right\rangle=\int_{g \in \Omega_{1} A_{T}^{+} \Omega_{2}} \psi(g) d g \\
& =\int_{k \in \Omega_{2}} \int_{1 \leq e^{r}<T} \int_{h \in \Omega_{1}} \psi\left(h a_{r} k\right) \rho\left(a_{r}\right) d h d r d k \\
& =\int_{k \in \Omega_{2}} \int_{T_{0} \leq e^{r}<T} \rho\left(a_{r}\right)\left(\int_{h \in \Omega_{1} \cdot X_{0}} \psi_{k}\left(h a_{r}\right) d h\right) d r d k+O_{T_{0}}(1) \\
& =\left(\frac{1}{\delta \cdot \mid m^{\mathrm{BMS} \mid}} \mu_{E}^{\mathrm{PS}}\left(\Omega_{1} X_{0}^{+}\right) \int_{k \in \Omega_{2}} m^{\mathrm{BR}}\left(\psi_{k}\right) d k+O(\epsilon)\right) \\
& \quad \times\left(\int_{0}^{\log T} e^{(r-n-1) \delta} \rho(r) d r\right)+O_{T_{0}}(1) \\
& =\frac{T^{\delta}}{\delta \cdot\left|m^{\mathrm{BMS}}\right|} \mu_{E}^{\mathrm{PS}}\left(\Omega_{1} X_{0}\right) \cdot m^{\mathrm{BR}}\left(\chi_{K^{*} \Omega_{2}} \psi\right)+O(\epsilon) T^{\delta}+O_{T_{0}}(1),
\end{aligned}
$$

where $\chi_{K} * \Omega_{2} \psi(g)=\int_{k \in \Omega_{2}} \psi(g k) d k$.

By the assumptions on $\Omega_{1}$ and $\Omega_{2}$, for every $\epsilon>0$ there exist $\epsilon$-neighborhoods $H_{\epsilon}$ and $K_{\epsilon}$ of $e$ in $H$ and $K$, respectively, such that for $\Omega_{1, \epsilon^{-}}:=\bigcap_{h \in H_{\epsilon}(H \cap M)} \Omega_{1} h$, $\Omega_{1, \epsilon^{+}}:=\Omega_{1} H_{\epsilon}(H \cap M), \Omega_{2, \epsilon^{-}}:=\bigcap_{k \in K_{\epsilon}} \Omega_{2} k$ and $\Omega_{2, \epsilon^{+}}:=\Omega_{2} K_{\epsilon}$, as $\epsilon \rightarrow 0$,

$$
\mu_{E}^{\mathrm{PS}}\left(\Omega_{1, \epsilon^{+}}\left(X_{0}\right) \backslash \Omega_{1, \epsilon^{-}}\left(X_{0}\right)\right) \rightarrow 0, \nu_{o}\left(\Omega_{2, \epsilon^{+}}^{-1}\left(X_{0}^{-}\right) \backslash \Omega_{2, \epsilon^{-}}^{-1}\left(X_{0}^{-}\right)\right) \rightarrow 0 .
$$

By Lemma 7.13, for $\ell>1$ as therein, there exists an $\epsilon$-neighborhood $U_{\epsilon}$ of $G$ such that for all $T \gg 1$,

$$
\begin{gathered}
\Omega_{1} A_{T^{+}}^{+} \Omega_{2} U_{\ell^{-1} \epsilon} \subset \Omega_{1, \epsilon^{+}} A_{(1+\epsilon) T}^{+} \Omega_{2, \epsilon^{+}}, \\
\Omega_{1, \epsilon^{-}} A_{(1-\epsilon) T^{+}}^{+} \Omega_{2, \epsilon^{-}} \subset \bigcap_{g \in U_{\ell^{-1} \epsilon}} \Omega_{1} A_{T}^{+} \Omega_{2} g .
\end{gathered}
$$

Let $\psi_{\epsilon} \in C_{c}(G)$ be a non-negative function supported on $U_{\ell^{-1} \epsilon}$ and $\int \psi_{\epsilon} d g=1$, and let $\Psi_{\epsilon} \in C_{c}(\Gamma \backslash G)$ be the $\Gamma$-average of $\psi_{\epsilon}$ :

$$
\Psi_{\epsilon}(g)=\sum_{\gamma \in \Gamma} \psi_{\epsilon}(\gamma g)
$$

It follows that

$$
\left\langle F_{(1-\epsilon) T, \Omega_{1, \epsilon^{-}}, \Omega_{2, \epsilon^{-}}}, \Psi_{\epsilon}\right\rangle \leq F_{T, \Omega_{1}, \Omega_{2}}(e) \leq\left\langle F_{(1+\epsilon) T, \Omega_{1, \epsilon}, \Omega_{2, \epsilon}+}, \Psi_{\epsilon}\right\rangle .
$$

On the other hand, by Proposition 7.5

$$
\lim _{\epsilon \rightarrow 0} m^{\mathrm{BR}}\left(\chi_{K} *_{\Omega_{2, \epsilon^{ \pm}}} \Psi_{\epsilon}\right)=\nu_{o}\left(\Omega_{2, \epsilon^{ \pm}}^{-1}\left(X_{0}^{-}\right)\right) .
$$

Therefore by (7.32),

$$
\lim _{T \rightarrow \infty} T^{-\delta}\left\langle F_{(1 \pm \epsilon) T, \Omega_{1, \epsilon^{ \pm}}, \Omega_{2, \epsilon^{ \pm}}}, \Psi_{\epsilon}\right\rangle=\frac{\mu_{E}^{\mathrm{PS}}\left(\Omega_{1, \epsilon^{ \pm}}\left(X_{0}\right)\right) \nu_{o}\left(\Omega_{2, \epsilon^{ \pm}}^{-1}\left(X_{0}^{-}\right)\right)}{\delta \cdot\left|m^{\mathrm{BMS}}\right|} .
$$

By (7.33) we get

$$
\lim _{T \rightarrow \infty} \frac{F_{T, \Omega_{1}, \Omega_{2}}(e)}{T^{\delta}}=\frac{1}{\delta \cdot\left|m^{\mathrm{BMS}}\right|} \mu_{E}^{\mathrm{PS}}\left(\Omega_{1, \epsilon^{+}}\left(X_{0}\right)\right) \nu_{o}\left(\Omega_{2, \epsilon^{+}}^{-1}\left(X_{0}^{-}\right)\right) .
$$


7.7. Counting theorems for $\Gamma$ Zariski dense. In the case when $\Gamma$ is Zariski dense, Theorem 7.8 holds for any norm on $V$ and for any $\Omega$ without the $M$ invariance condition. Similarly, Theorem $[7.16$ holds without the $M$-invariance assumption on $\Omega_{1}$ and $\Omega_{2}$.

The reason that this generalization is possible is because for $\Gamma$ Zariski dense, we use Theorem 7.7 instead of Theorem 1.8. In proving Theorem 7.8, the place where we needed the $M$-invariance of $\Omega$ is in Proposition 7.11 For general $\Omega$, we replace this proposition by

$$
\begin{aligned}
& \int_{k \in \Omega} \int_{0}^{r_{-}(k, T)} \rho(r)\left(\int_{\Gamma_{w_{0}} \backslash H} \psi_{k}\left(h a_{r}\right) d h\right) d r d k \\
& \leq\left\langle F_{B_{T}(\Omega)}, \psi\right\rangle \leq \int_{k \in \Omega} \int_{0}^{r_{+}(k, T)} \rho(r)\left(\int_{\Gamma_{w_{0}} \backslash H} \psi_{k}\left(h a_{r}\right) d h\right) d r d k,
\end{aligned}
$$

where $\psi_{k}(g):=\psi(g k) \in C_{c}(\Gamma \backslash G)$ is simply the translation of $\psi$ by $k$.

Applying Theorem 7.7 to the inner integral in the above, we deduce in the same way as in the proof of Proposition 7.12 that for any $\psi \in C_{c}(\Gamma \backslash G)$, we have

$$
\lim _{T \rightarrow \infty} T^{-\delta / \lambda}\left\langle F_{B_{T}(\Omega)}, \psi\right\rangle=\frac{\mu_{E}^{\mathrm{PS}}\left(E^{*}\right)}{\delta \cdot\left|m^{\mathrm{BMS}}\right|} \cdot \bar{m}^{\mathrm{BR}}\left(\xi_{w_{0}} * \Omega \psi\right),
$$

where $\bar{m}^{\mathrm{BR}}$ is defined as in $\oint 7.3$ and $\xi_{w_{0}}(k):=\left\|w_{0}^{\lambda} k\right\|^{-\delta / \lambda}$. Now for a general norm $\|\cdot\|$ on $V$, note that the function $\xi_{w_{0}}(k)$ is not necessarily $M$-invariant. However, for an approximate identity $\left\{\psi_{\epsilon}\right\}_{\epsilon>0}$ on $G$ and any $f \in C(K)$, the proof of Proposition 7.5 can be easily modified to prove

$$
\lim _{\epsilon \rightarrow 0} \bar{m}^{\mathrm{BR}}\left(f *_{\Omega} \psi_{\epsilon}\right)=\int_{k \in \Omega^{-1}} f\left(k^{-1}\right) d \nu_{o}\left(k X_{0}^{-}\right) .
$$

Hence applying (7.34) to $\psi=\psi_{\epsilon}$ and (7.35) to $f=\xi_{w_{0}}$ and by sending $\epsilon \rightarrow 0$, we obtain

$$
\lim _{T \rightarrow \infty} T^{-\delta / \lambda} \cdot F_{B_{T}(\Omega)}(e)=\frac{\mu_{E}^{\mathrm{PS}}\left(E^{*}\right)}{\delta \cdot\left|m^{\mathrm{BMS}}\right|} \cdot \int_{k \in \Omega^{-1}}\left\|w_{0}^{\lambda} k^{-1}\right\|^{-\delta / \lambda} d \nu_{o}\left(k X_{0}^{-}\right) .
$$

This explains the generalization of Theorem $7.8(1)$. The generalization for Theorem 7.8(2) and Theorem 7.16 can be done similarly.

Proof of Theorem 7.10, In view of the above explanation, the result can be deduced from Theorem 7.8 (or its combination with Proposition 7.14 or Remark 7.15) via elementary arguments; see [13.

\section{Appendix: Equality of two HaAr measures}

Let $H$ be a symmetric group as in 7.2 .1 . As in Notation [7.4(1), consider the Haar measure on $G$ corresponding to the Iwasawa decomposition $G=N A K$ given by

$$
d g=e^{(n-1) t} d n d t d q, \text { for } g=n a_{t} q, n \in N, a_{t} \in A, q \in K .
$$

Corresponding to the generalized Cartan decomposition $G=H A K$, by (7.8) the Haar measure on $G$ can be expressed as

$$
d g=c_{0} \cdot \rho(r) d h d r d k, \text { for } g=h a_{r} k \in H A K,
$$

where $c_{0}>0$ is a constant. We note that $d n$ is defined by Lemma 7.1 and $d h$ is determined by (7.7). 
Theorem 8.1. $c_{0}=1$.

Proof. Let the notation be as in 97.1 . Let $N^{-}=\left\{g \in G: a_{-r} g a_{r} \rightarrow e\right.$ as $\left.r \rightarrow \infty\right\}$. Then for $y \in \operatorname{Lie}\left(N^{-}\right)$we have $a_{-r} \exp (y) a_{r}=\exp \left(e^{-r} y\right)$. In view of an $N A N^{-} M$ decomposition of a small neighborhood of $e$ in $G$, for $h$ in such a neighborhood we write

$$
h=n(x(h)) a_{b(h)} v(y(h)) m(h),
$$

where $x(h) \in \operatorname{Lie}(N) \cong \mathbb{R}^{n-1}$ and $n(x(h))=\exp (x(g)), y(h) \in \operatorname{Lie}\left(N^{+}\right) \cong \mathbb{R}^{n-1}$ and $v(y(h))=\exp (y(h)), b(h) \in \mathbb{R}$ and $m(h) \in M$. In particular,

$$
h X_{0}^{+}=n(x(h)) a_{b(h)} v(y(h)) m(h) X_{0}^{+}=n(x(h)) X_{0}^{+}
$$

In view of the decompositions $G=H A K$ and $G=N A K$, for $h \in H, r>0$ and $k \in K$, we express

$$
h a_{r} k=n(z(h, r, k)) a_{t(h, r, k)} q(h, r, k), \text { where } q(h, r, k) \in K .
$$

Now for $h$ in a small neighborhood of $e$ in $H$, we have

$$
h a_{r} k=n(x(h)) a_{b(h)} v(y(h)) m(h) a_{r} k=n(x(h)) a_{r+b(h)} v\left(e^{-r} y(h)\right)(m(h) k) .
$$

In view of a $G=N A K$ decomposition,

$$
\begin{gathered}
v\left(e^{-r} y(h)\right)=n\left(x_{1}(h, r)\right) a_{b_{1}(h, r)} k_{1}(h, r), \text { with } \\
\max \left(\left\|x_{1}(h, r)\right\|,\left\|b_{1}(h, r)\right\|,\left\|k_{1}(h, r)\right\|\right)=O\left(e^{-r}\|x(h)\|\right) .
\end{gathered}
$$

Therefore,

$$
\begin{aligned}
h a_{r} k & =n(x(h)) a_{r+b(h)} n\left(x_{1}(h, r)\right) a_{b_{1}(h, r)}\left(k_{1}(h, r) m(h) k\right) \\
& =n\left(x(h)+x_{2}(h, r)\right) a_{r+b(h)+b_{1}(h, r)}\left(k_{1}(h, r) m(h) k\right),
\end{aligned}
$$

where $x_{2}(h, r)=e^{-r-b(h)} x_{1}(h, r)$. So

$$
\left\|x_{2}(h, r)\right\|=e^{-2 r} O(\|x(h)\|) .
$$

Therefore

$$
\begin{aligned}
& z(h, r, k)=n\left(x(h)+x_{2}(h, r)\right), \quad t(h, r, k)=r+b(h)+b_{1}(h, r), \\
& q(h, r, k)=k_{1}(h, r) m(h) k .
\end{aligned}
$$

Since $z(h, r, k)=z(h m, r, e)$ and $t(h, r, k)=t(h m, r, e)$ for any $k \in K$ and $m \in$ $M \cap H=G_{X_{0}^{+}} \cap H$, we can write $z(h, r, k)=z([h], r)$ and $t([h], r, k)=t([h], r)$, where $[h]=h(M \cap H)=h X_{0}^{+}$. Moreover, for any fixed $h$ and $r$, since $d k$ is $K$-invariant, we have that $d q(h, r, k)=d k$.

For $h$ in a small neighborhood of $e$ in $H, r>0$ and $k \in K$,

$$
\begin{aligned}
c_{0} & =\frac{e^{(n-1) t(h, r, k)} d n(z(h, r, k)) d t(h, r, k) d q(h, r, k)}{\rho(r) d h d r d k} \\
& =\frac{e^{(n-1) t([h], r)} d n(z([h], r)) d t([h], r)}{\rho(r) d h d r} \cdot \frac{d q(h, r, k)}{d k} \\
& =\frac{e^{(n-1) t([h], r)} d n(z([h], r)) d t([h], r)}{\rho(r) d h d r}
\end{aligned}
$$


because $z$ and $t$ do not depend on $k$ and for fixed $(h, r)$ we have $d(q(h, r, k))=d k$. Now the numerator depends only on $[h]=h M$ and $\int_{m \in H \cap M} 1 d m=1$. Therefore,

$$
\begin{aligned}
c_{0}=\frac{e^{(n-1) t([h], r)} e^{(n-1) \beta_{n(z([h], r)) X_{0}^{+}}(o, n(z(h, r, k)) o)}}{\rho(r) e^{(n-1) \beta_{[h]}(o,[h] o)}} & \\
& \times \frac{d m_{o}\left(n(z([h], r)) X_{0}^{+}\right) d t([h], r)}{d m_{o}([h]) d r} .
\end{aligned}
$$

To compute $c_{0}$, we evaluate the Radon-Nikodym derivative at the point $([h], r)=$ $([e], s)=\left(X_{0}^{+}, s\right)$ for any fixed $s>0$. Then we consider the upper half space model $\mathbb{R}^{n-1} \times \mathbb{R}_{>0}$ for $\mathbb{H}^{n}$ with $o=(0,1)$ and $X_{0}^{-}=\infty$. Then $X_{0}^{+}=0 \in \mathbb{R}^{n-1}=$ $\partial \mathbb{H}^{n} \backslash\{\infty\}$. Since $m_{o}$ is equivalent to the Lebesgue measure, let

$$
0<C:=\left.\frac{d m_{o}(x)}{d x}\right|_{x=0} ; \text { also } n(x) X_{0}^{+}=x \text { for all } x \in \mathbb{R}^{n-1} .
$$
by

We define a map $\Phi$ from a small neighborhood of $(0, s)$ in $\mathbb{R}^{n-1} \times \mathbb{R}$ to $\mathbb{R}^{n-1} \times \mathbb{R}$

$$
\Phi([h], r)=\left(n\left(z(h, r, k) X_{0}^{+}, t([h], r)\right)\right) .
$$

To compute the Jacobian of $\Phi$ at the point $\left(X_{0}^{+}, s\right)=(0, s)$, we write $\Phi=\left(\Phi_{1}, \Phi_{2}\right)$ and $([h], r)=\left(z_{1}, z_{2}\right)$.

Fixing $[h]=[e]$, we get $z([e], r)=0, t([e], r)=r$. Therefore $\partial_{z_{2}}\left(\Phi_{1}, \Phi_{2}\right)=(0,1)$. Hence the Jacobian of $\Phi$ at $([h], r)=(0, s)$ is

$$
\begin{aligned}
& J(\Phi)(0, s)=\left|\partial_{z_{1}} \Phi_{1}(0, s)\right| \\
& =\frac{d m_{o}\left(n\left(x_{2}([h], s)+x(h)\right) X_{0}^{+}\right)}{d m_{o}([h])} \text { at }[h]=0 \text {, by (8.3) } \\
& =\frac{d m_{o}\left(n\left(x_{2}([h], s)+x([h])\right) X_{0}^{+}\right)}{d m_{o}\left(n(x([h])) X_{0}^{+}\right)} \text {, by (8.1) } \\
& =\frac{d\left(x_{2}([h], s)+x([h])\right)}{d(x([h]))} \text { at }[h]=0=x([h]) \text {, by (8.5]) } \\
& =1+\frac{d\left(x_{2}([h], s)\right)}{d(x([h]))} \text { at }[h]=0=x([h]) \\
& =1+O\left(e^{-2 s(n-1)}\right) \text {, by (8.2). }
\end{aligned}
$$

Note that for a fixed $s$, due to (8.1) and (8.5), $x_{2}([h], s)$ is a smooth function of $x([h])$. By (8.4), the Radon-Nikodym derivative at $([h], r)=([e], s)$ is

$$
\begin{aligned}
c_{0} & =\frac{e^{(n-1) t([e], s)} e^{(n-1) \beta_{n(z([e], s)) X_{0}^{+}}(o, n(z([e], s)) o)}}{\rho(s) e^{(n-1) \beta_{[e]}(o,[e] o)}} \cdot J(\Phi)(0, s) \\
& =\left(e^{(n-1) s} / \rho(s)\right)\left(1+O\left(e^{-2 s(n-1)}\right)\right) .
\end{aligned}
$$

Since $\rho(s) / e^{(n-1) s} \rightarrow 1$ as $s \rightarrow \infty$, we have $c_{0}=1$.

\section{ACKNOWLEDGEMENTS}

The authors thank Thomas Roblin for useful comments on an earlier version of this paper. The authors also thank the referee for carefully reading the paper and asking many pertinent questions, which led us to proving more general results and improving the overall presentation of the paper. 


\section{REFERENCES}

[1] Martine Babillot. On the mixing property for hyperbolic systems. Israel J. Math., 129:61-76, 2002. MR:1910932 (2003g:37008)

[2] Alan F. Beardon. The Geometry of Discrete Groups, volume 91 of Graduate Texts in Mathematics. Springer-Verlag, New York, 1983. MR698777 (85d:22026)

[3] Yves Benoist and Hee Oh. Effective equidistribution of $S$-integral points on symmetric varieties To appear in Annales de L'Institut Fourier, arXiv:0706.1621.

[4] B. H. Bowditch. Geometrical finiteness for hyperbolic groups. J. Funct. Anal., 113(2):245317, 1993. MR1218098 (94e:57016)

[5] Rufus Bowen. Periodic points and measures for Axiom A diffeomorphisms. Trans. Amer. Math. Soc., 154:377-397, 1971. MR0282372(43:8084)

[6] Marc Burger. Horocycle flow on geometrically finite surfaces. Duke Math. J., 61(3):779-803, 1990. MR1084459 (91k:58102)

[7] F. Dal'bo. Topologie du feuilletage fortement stable. Ann. Inst. Fourier (Grenoble), 50(3):981-993, 2000. MR 1779902 (2001i:37045)

[8] F. Dal'bo and J. P. Otal and M. Peigné. Séries de Poincaré des groupes géométriquement finis. Israel J. Math., 118, 109-124, 2000. MR1776078 (2001g:37040)

[9] W. Duke, Z. Rudnick, and P. Sarnak. Density of integer points on affine homogeneous varieties. Duke Math. J., 71(1):143-179, 1993. MR.1230289 (94k:11072)

[10] Alex Eskin and C. T. McMullen. Mixing, counting, and equidistribution in Lie groups. Duke Math. J., 71(1):181-209, 1993. MR.1230290(95b:22025)

[11] Livio Flaminio and Ralf Spatzier. Geometrically finite groups, Patterson-Sullivan measures and Ratner's ridigity theorem. Invent Math., 99:601-626, 1990. MR1032882 (91d:58201)

[12] Alex Gorodnik and Hee Oh. Orbits of discrete subgroups on a symmetric space and the Furstenberg boundary. Duke Math. J., 139(3):483-525, 2007. MR2350851 (2008k:22020)

[13] Alex Gorodnik, Nimish Shah, and Hee Oh. Integral points on symmetric varieties and Satake compactifications. Amer. J. Math., 131(1): 1-57, 2009. MR2488484(2009m:22017)

[14] Alex Gorodnik, Nimish Shah, and Hee Oh. Strong wavefront lemma and counting lattice points in sectors. Israel J. Math, 176:419-444, 2010. MR.2653201 (2011h:22009)

[15] D. Y. Kleinbock and G. A. Margulis. Bounded orbits of nonquasiunipotent flows on homogeneous spaces. In Sinaŭ's Moscow Seminar on Dynamical Systems, volume 171 of Amer. Math. Soc. Transl. Ser. 2, pages 141-172. Amer. Math. Soc., Providence, RI, 1996. MR1359098 (96k:22022)

[16] Alex Kontorovich and Hee Oh. Almost prime Pythagorean triples in thin orbits. To appear in Crelle, arXiv:1001.0370.

[17] Alex Kontorovich and Hee Oh. Apollonian circle packings and closed horospheres on hyperbolic 3-manifolds; with appendix by Oh and Shah. Journal of AMS, 24 (2011) 603-648. MR2784325

[18] Steven P. Lalley. Renewal theorems in symbolic dynamics, with applications to geodesic flows, non-Euclidean tessellations and their fractal limits. Acta Math., 163(1-2):1-55, 1989. MR.1007619 (91c:58112)

[19] Peter D. Lax and Ralph S. Phillips. The asymptotic distribution of lattice points in Euclidean and non-Euclidean spaces. J. Funct. Anal., 46(3):280-350, 1982. MR661875 (83j:10057)

[20] Gregory Margulis. On some aspects of theory of Anosov systems. Springer Monographs in Mathematics. Springer-Verlag, Berlin, 2004. MR2035655 (2004m:37049)

[21] Bernard Maskit. Kleinian groups. Springer-Verlag, Berlin, 1988. MR959135 (90a:30132)

[22] Hee Oh. Dynamics on geometrically finite hyperbolic manifolds with applications to Apollonian circle packings and beyond. Proc. of I.C.M, Hyderabad, Vol. III, 1308-1331, 2010. MR2827842(2012f:37004)

[23] Hee Oh and Nimish Shah. The asymptotic distribution of circles in the orbits of Kleinian groups. Inventiones Math., Vol. 187, 1-35, 2012. MR2874933

[24] Hee Oh and Nimish Shah. Counting visible circles on the sphere and Kleinian groups. Preprint, arXiv:1004.2129.

[25] Hee Oh and Nimish Shah. Limits of translates of divergent geodesics and integral points on one-sheeted hyperboloids. Preprint, arXiv:1104.4988.

[26] S.J. Patterson. The limit set of a Fuchsian group. Acta Mathematica, 136:241-273, 1976. MR.0450547(56:8841) 
[27] Marc Peigné. On the Patterson-Sullivan measure of some discrete group of isometries. Israel J. Math., 133:77-88, 2003. MR 1968423(2004b:30079)

[28] B. Randol. The behavior under projection of dilating sets in a covering space. Trans. Amer. Math. Soc., 285:855-859, 1984. MR752507 (86g:58023)

[29] M. S. Raghunathan. Discrete subgroups of Lie groups. Springer-Verlag, New York, 1972. Ergebnisse der Mathematik und ihrer Grenzgebiete, Band 68. MR0507234 (58:22394a)

[30] Marina Ratner. On Raghunathan's measure conjecture. Ann. of Math. (2), volume 134, 1991. MR.1135878(93a:22009)

[31] Thomas Roblin. Ergodicité et équidistribution en courbure négative. Mém. Soc. Math. Fr. (N.S.), (95):vi+96, 2003. MR2057305 (2005d:37060)

[32] Daniel J. Rudolph. Ergodic behaviour of Sullivan's geometric measure on a geometrically finite hyperbolic manifold. Ergodic Theory Dynam. Systems, 2(3-4):491-512, 1983. MR721736 (85i:58101)

[33] Peter Sarnak. Asymptotic behavior of periodic orbits of the horocycle flow and eisenstein series. Comm. Pure Appl. Math., 34(6):719-739, 1981. MR634284 (83m:58060)

[34] Barbara Schapira. Equidistribution of the horocycles of a geometrically finite surface. Int. Math. Res. Not., (40):2447-2471, 2005. MR2180113 (2006i:37073)

[35] H. Schlichtkrull. Hyperfunctions and Harmonic Analysis on Symmetric Spaces, Progress in Mathematics, 49. Birkhaüser Boston, Inc., Boston, MA, 1984. MR757178 (86g:22021)

[36] Dennis Sullivan. The density at infinity of a discrete group of hyperbolic motions. Inst. Hautes Études Sci. Publ. Math., (50):171-202, 1979. MR556586 (81b:58031)

[37] Dennis Sullivan. Entropy, Hausdorff measures old and new, and limit sets of geometrically finite Kleinian groups. Acta Math., 153(3-4):259-277, 1984. MR766265 (86c:58093)

[38] S. T. Yau. Harmonic functions on complete Riemannian manifolds. Comm. Pure. Appl. Math., (28):201-228, 1975. MR0431040 (55:4042)

Department of Mathematics, Brown University, Providence, Rhode Island 02912 and Korea Institute for Advanced Study, Seoul, Korea

E-mail address: heeoh@math.brown.edu

Department of Mathematics, The Ohio State University, Columbus, Ohio 43210

E-mail address: shah@math.ohio-state.edu 